\title{
FINITE ELEMENT MODELING OF DELAMINATION DAMAGE IN CARBON FIBER LAMINATES SUBJECT TO LOW-VELOCITY IMPACT AND COMPARISON WITH EXPERIMENTAL IMPACT TESTS USING NONDESTRUCTIVE VIBROTHERMOGRAPHY EVALUATION
}

\author{
A Thesis \\ presented to \\ the Faculty of California Polytechnic State University, \\ San Luis Obispo
}

\author{
In Partial Fulfillment \\ of the Requirements for the Degree \\ Master of Science in Mechanical Engineering
}

by

George Rodriguez

June 2016 
(C) 2016

George Rodriguez

ALL RIGHTS RESERVED 
TITLE:

AUTHOR:

DATE SUBMITTED:

COMMITTEE CHAIR:

COMMITTEE MEMBER:

COMMITTEE MEMBER:
Finite Element Modeling of Delamination Damage in Carbon Fiber Laminates Subject to Low-Velocity Impact and Comparison with Experimental Impact Tests Using Nondestructive Vibrothermography Evaluation

George Rodriguez

June 2016

Thomas Mase, Ph.D.

Professor of Mechanical Engineering

Joseph Mello, Ph.D.

Professor of Mechanical Engineering

John Chen, Ph.D.

Professor of Mechanical Engineering 


\begin{abstract}
Finite Element Modeling of Delamination Damage in Carbon Fiber Laminates Subject to Low-Velocity Impact and Comparison with Experimental Impact Tests Using Nondestructive Vibrothermography Evaluation

George Rodriguez
\end{abstract}

Carbon fiber reinforced composites are utilized in many design applications where high strength, low weight, and/or high stiffness are required. While composite materials can provide high strength and stiffness-to-weight ratios, they are also more complicated to analyze due to their inhomogeneous nature. One important failure mode of composite structures is delamination. This failure mode is common when composite laminates are subject to impact loading.

Various finite element methods for analyzing delamination exist. In this research, a modeling strategy based on contact tiebreak definitions in LS-DYNA ${ }^{\circledR}$ was used. A finite element model of a low-velocity impact event was created to predict delamination in a composite laminate. The resulting delamination relative size and shape was found to partially agree with analytical and experimental results for similar impact events, while the force-time plot agreed well with experimental results. A small difference in contact time in the simulation compared to experimental testing is likely due to the omission of composite failure modes other than delamination.

Experimental impact testing and subsequent vibrothermography analysis showed delamination damage in locations shown in previous research. This confirmed the validity of vibrothermography as a nondestructive evaluation technique for analyzing post-impact delamination. 


\section{ACKNOWLEDGMENTS}

I would like to thank my family for their unwavering love and support. Any achievements of mine are just as much their achievements as they are mine. I am extremely grateful to have had the chance to attend Cal Poly and learn from the highly intelligent faculty. Specifically, I would like to thank the members of my thesis committee. My first engineering course began with Dr. Chen in Statics and helped set the foundation for my engineering education. His knowledge of vibrothermography and composite impact testing was crucial to the experimental portion of my research. Dr. Mase allowed me to work under him early in my undergraduate career and has shaped my future with his knowledge and advice. His knowledge of LS-DYNA ${ }^{\circledR}$ made my thesis possible. Dr. Mello has put up with me in four separate courses and inspired my passion for finite element modeling and structural mechanics. His investment in his students and real world teaching style is the epitome of Cal Poly's engineering philosophy.

I would also like to thank my fellow graduate students Joseph Garrett and Michael Schuster. Joseph and I spent countless hours together bouncing ideas off each other and making composite specimens, while Michael's experimental testing expertise saved me many hours of troubleshooting my experiments. 


\section{TABLE OF CONTENTS}

Page

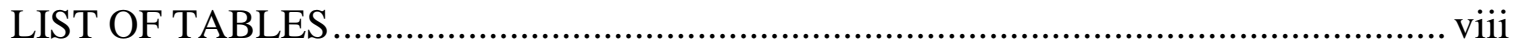

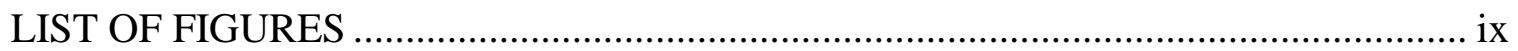

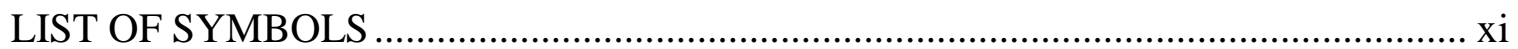

CHAPTER

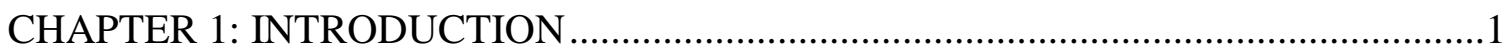

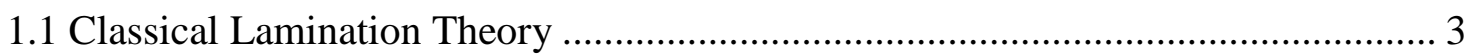

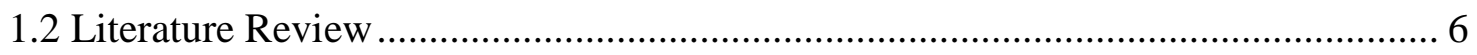

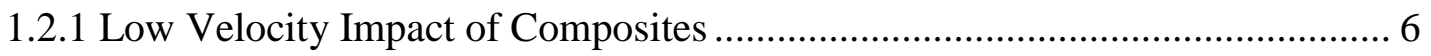

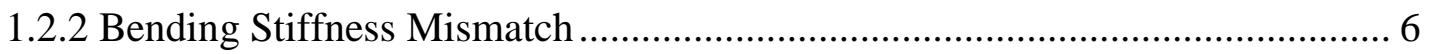

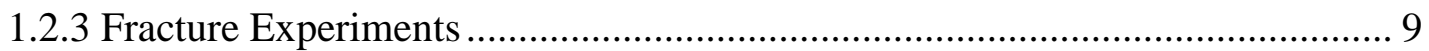

1.2.4 Delamination Modeling Strategies ........................................................ 11

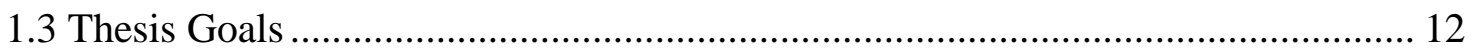

CHAPTER 2: FINITE ELEMENT MODELING …............................................ 14

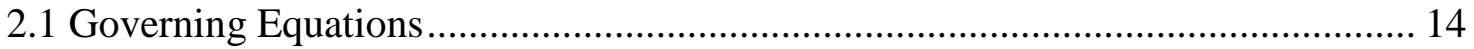

2.2 Mode I Delamination: Double Cantilever Beam................................................ 15

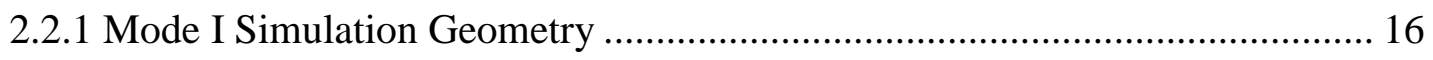

2.2.2 Contact Tiebreak Used in Mode I Simulation ............................................. 17

2.2.3 Finite Element Parameters ............................................................................ 21

2.2.3.1 Mesh \& Contact Tiebreak Mesh Dependency ....................................... 21

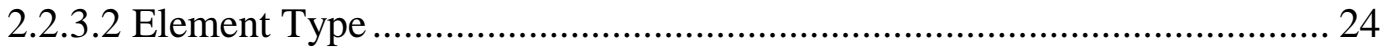

2.2.3.3 Material Constitutive Response ..................................................... 25

2.2.3.4 Boundary Conditions for Mode I Analysis ........................................... 26

2.2.3.5 Mass and Stiffness Damping Considerations ....................................... 26

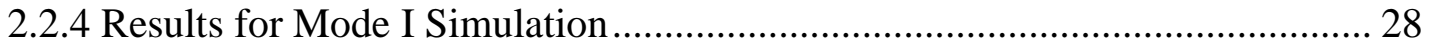

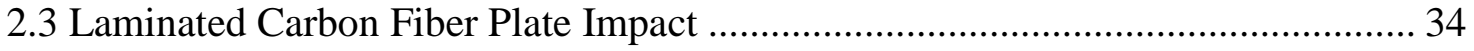

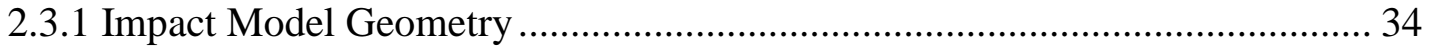

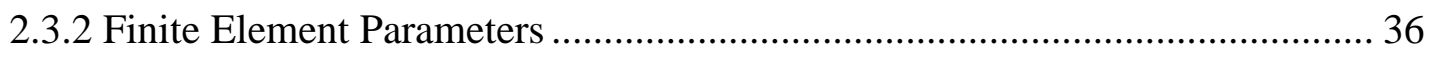

2.3.2.1 Meshing \& Contact Tiebreak.............................................................. 36

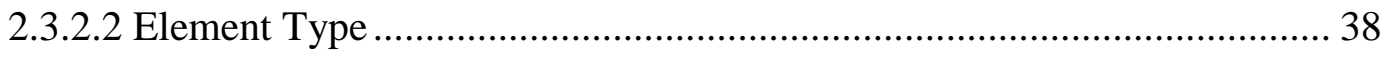

2.3.2.3 Material Constitutive Response ........................................................... 38 


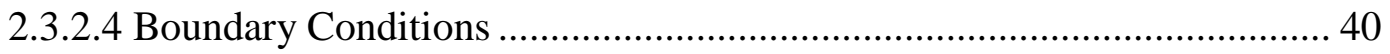

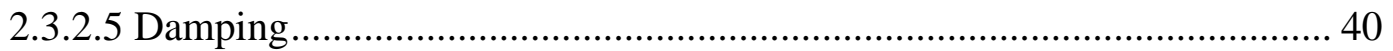

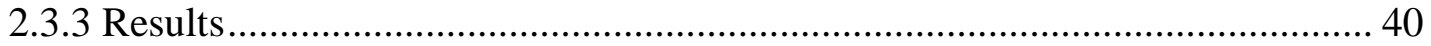

CHAPTER 3: EXPERIMENTAL IMPACT TESTING ...............................................48

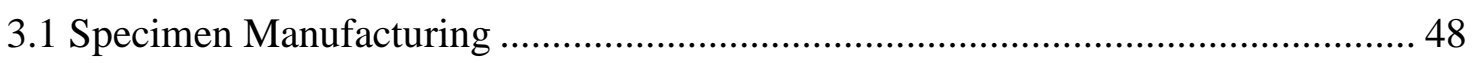

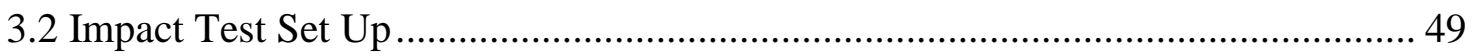

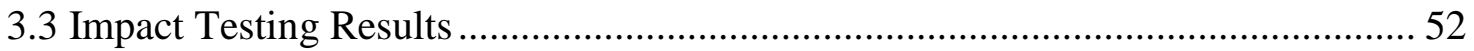

CHAPTER 4: VIBROTHERMOGRAPHY EXPERIMENTAL TESTING ………….......55

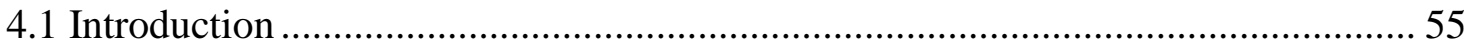

4.2 Vibrothermography Experimental Test Set Up ................................................... 56

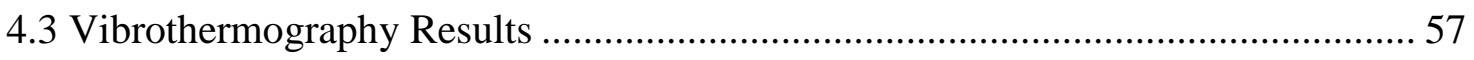

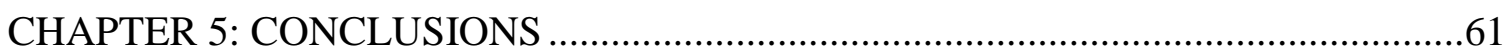

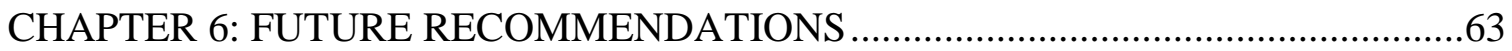

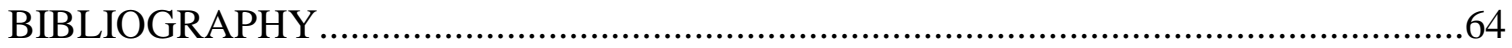

APPENDICES

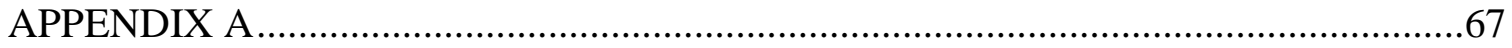

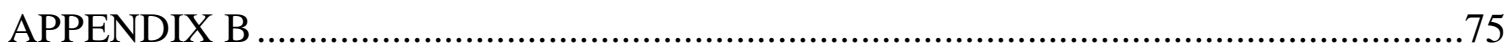

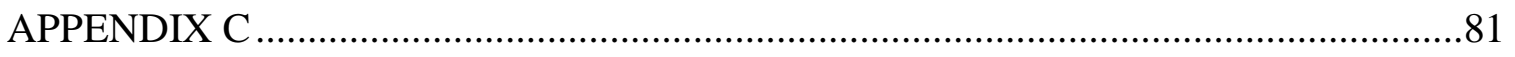

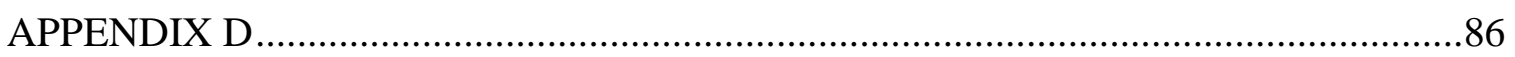

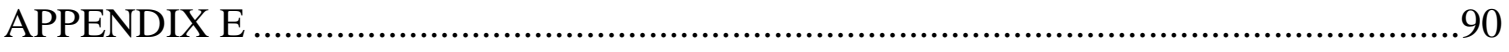

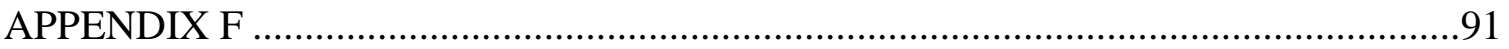




\section{LIST OF TABLES}

Table

Page

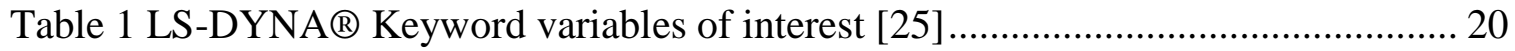

Table 2 Values used for contact tiebreak variables in Mode I simulation ....................... 23

Table 3 Material properties for orthotropic Material Type 2 in LS-DYNA ${ }^{\circledR}[19] \ldots \ldots \ldots . . .26$

Table 4 M46J/TC250 orthotropic material properties in LS-DYNA ${ }^{\circledR}$............................. 39

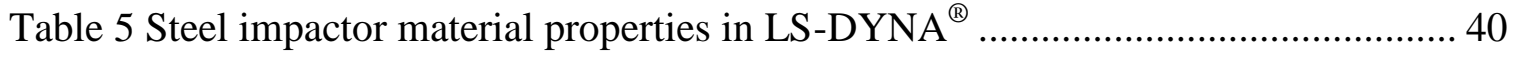

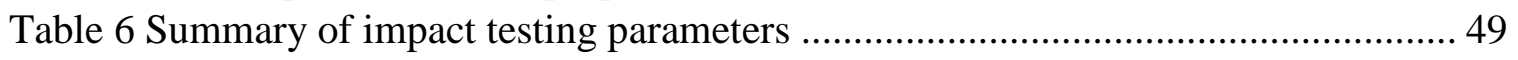


Figure

\section{LIST OF FIGURES}

Figure 1 Example of delamination in a laminated composite [1] ..................................... 2

Figure 2 Bending stiffness mismatch plot ............................................................ 8

Figure 3 Back ply orientation effect on predicted delamination for $\left[-30_{\alpha} / 60_{\alpha}\right]$ laminate ... 9

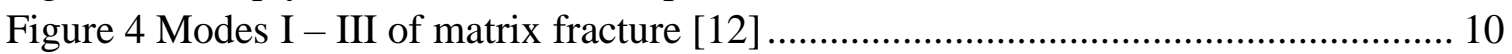

Figure 5 LS-DYNA ${ }^{\circledR}$ Mode I DCB FE simulation geometry and mesh........................... 16

Figure 6 Pre-cracked length for Mode I DCB simulation............................................... 17

Figure 7 Bilinear traction-separation law for *MAT_138 ........................................... 19

Figure 8 Mixed-mode traction-separation law.............................................................. 21

Figure 9 Bottom sublaminate pre-cracked segments with automatic contact................... 23

Figure 10 Bottom sublaminate contact tiebreak segments ............................................. 23

Figure 11 Portion of sublaminate used in modal analysis ............................................. 27

Figure 12 First natural frequency mode for the double cantilever beams in bending ...... 28

Figure 13 Force-displacement response for similar DCB simulation by Kay [19] .......... 29

Figure 14 Crack opening force vs. crack opening distance for DCB specimen ............... 30

Figure 15 Rate dependency of DCB response prior to and after crack propagation ........ 31

Figure 16 Rate dependency of DCB response up to crack propagation ............................ 31

Figure 17 Peak traction stress effects on Mode I DCB force-displacement response...... 32

Figure 18 Mass damping effect on DCB response prior to and after crack propagation.. 33

Figure 19 Mass damping effect on DCB response up to crack propagation .................... 33

Figure 20 Laminated carbon fiber plate impact model: side view..................................... 35

Figure 21 Laminated carbon fiber plate impact model: isometric view ........................... 35

Figure 22 Aspect ratio of solid elements in impact model ............................................... 37

Figure 23 Maximum distortion angle of solid elements in impact model ....................... 37

Figure 24 Composite material directions [32] .............................................................. 39

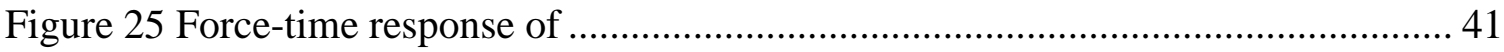

Figure 26 Delamination failure between sublaminates in $\mathrm{x}$-y plane................................ 43

Figure 27 Delamination failure between sublaminates in y-z plane................................. 43

Figure 28 Contour plot of separation damage between top and mid sublaminates .......... 44

Figure 29 Contour plot of shear separation damage between top \& mid sublaminates ... 45

Figure 30 Contour plot of normal separation damage between mid \& bot sublaminates. 45

Figure 31 Contour plot of shear separation damage between mid \& bot sublaminates ... 46

Figure $32 \mathrm{X}$-ray of T300/976 $\left[00_{4} / 90_{4}\right]_{\mathrm{s}}$ plate quasi-impact loaded with $13.4 \mathrm{lb}_{\mathrm{f}}$-in [5] ... 47

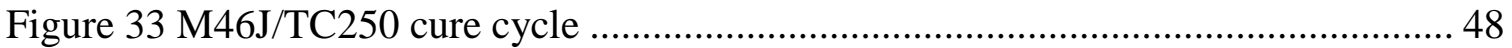

Figure 34 Instron Dynatup 8250 drop weight impact machine ………............................. 50

Figure 35 Dynatup 8250 clamping fixture.................................................................. 51

Figure 36 Contact force: $12.2 \mathrm{lb}_{\mathrm{f}}$-in impact energy on $\left[0_{4} / 90_{4}\right]_{\mathrm{s}} \mathrm{M} 46 \mathrm{~J} / \mathrm{TC} 250$ laminate.. 53

Figure 37 Contact force: $15.7 \mathrm{lb}_{\mathrm{f}}$-in impact energy on $\left[0_{4} / 90_{4}\right]_{\mathrm{s}}$ M46J/TC250 laminate.. 53

Figure 38 Contact force: $19.2 \mathrm{lb}_{\mathrm{f}}$-in impact energy on $\left[0_{4} / 90_{4}\right]_{\mathrm{s}} \mathrm{M} 46 \mathrm{~J} / \mathrm{TC} 250$ laminate.. 54

Figure 39 Contact force: $27.9 \mathrm{lb}_{\mathrm{f}}$-in impact energy on $\left[0_{4} / 90_{4}\right]_{\mathrm{s}}$ M46J/TC250 laminate.. 54

Figure $40 \mathrm{~S}-2$ glass/epoxy $\left[0_{10} / 90_{10}\right]$ laminate, $32.4 \mathrm{lbf}$-in impact energy ...................... 55

Figure 41 Experimental setup for exciting specimen and viewing heat generation ..........56 
Figure 42 Clamped $\left[0_{4} / 90_{8} / 0_{4}\right]$ composite specimen

Figure 43 Stacking sequence for impacted laminate tested using vibrothermography .... 58

Figure 44 Clamped specimen stacking sequence with predicted delamination shapes .... 60

Figure 45 Vibrothermography IR images: backside view (left) and impact side (right).. 60

Figure 46 Overlaid experimental acceleration data for various impact energies............. 90

Figure 47 Backside view of $\left[0_{4} / 90_{8} / 0_{4}\right]$ laminate from $12.2 \mathrm{lb}$-in impact energy ........... 91

Figure 48 Impact side view of $\left[0_{4} / 90_{8} / 0_{4}\right]$ laminate from $12.2 \mathrm{lb}$-in impact energy ....... 91

Figure 49 Backside view of $\left[00_{4} / 90_{8} / 0_{4}\right]$ laminate from $15.7 \mathrm{lb}_{\mathrm{f}}$-in impact energy ............ 92

Figure 50 Impact side view of $\left[0_{4} / 90_{8} / 0_{4}\right]$ laminate from $15.7 \mathrm{lb}_{\mathrm{f}}$-in impact energy ....... 92

Figure 51 Backside view of $\left[00_{4} / 90_{8} / 0_{4}\right]$ laminate from $19.2 \mathrm{lb}_{\mathrm{f}}$-in impact energy ........... 93

Figure 52 Impact side view of $\left[0_{4} / 90_{8} / 0_{4}\right]$ laminate from $19.2 \mathrm{lb}_{\mathrm{f}}$-in impact energy ........ 93

Figure 53 Backside view of $\left[0_{4} / 90_{8} / 0_{4}\right]$ laminate from $27.9 \mathrm{lb}_{\mathrm{f}}$-in impact energy ........... 94

Figure 54 Impact side view of $\left[0_{4} / 90_{8} / 0_{4}\right]$ laminate from $27.9 \mathrm{lb}_{\mathrm{f}}$-in impact energy ....... 94 


\section{LIST OF SYMBOLS}

$\begin{array}{ll}\sigma & \text { Stress } \\ \tau & \text { Shear stress } \\ E & \text { Young's modulus } \\ C & \text { Compliance constants } \\ \varepsilon & \text { Normal strain } \\ \gamma & \text { Shear strain }\end{array}$

[Q] Material coordinate stiffness matrix

$N$ Resultant normal/shearing forces in CLT

$M \quad$ Resultant bending/twisting moments in CLT

$\kappa \quad$ Curvature

$A_{i j} \quad$ Resultant laminate force-strain stiffness matrix

$B_{i j} \quad$ Resultant laminate force-curvature stiffness matrix

$D_{i j} \quad$ Resultant laminate moment-curvature stiffness matrix

$Z \quad$ Distance from laminate mid-plane to lamina mid-plane

$M_{b} \quad$ Bending stiffness mismatch parameter

$\theta \quad$ Ply orientation

[M] Mass matrix

[C] Damping coefficient matrix

$[K] \quad$ Stiffness matrix

$a_{0} \quad$ Pre-cracked delamination length

$G_{c} \quad$ Fracture energy release rate

$\delta_{m} \quad$ Separation parameter for contact tiebreak definition in LS-DYNA ${ }^{\circledR}$

$\delta^{0} \quad$ Mixed-mode damage initiation distance

$\delta^{F} \quad$ Ultimate mixed-mode displacement for complete failure

$\beta \quad$ Mode mixity for traction-separation law

$c_{c r} \quad$ Critical mass damping coefficient 


\section{CHAPTER 1: INTRODUCTION}

Carbon fiber reinforced composites are utilized in many design applications where high strength, low weight, and/or high stiffness are required. From the aerospace industry to the commercial golf club industry, carbon fiber reinforced composites have become the material of choice for many designs. While composite materials can provide high strength and stiffness-to-weight ratios, they are also more complicated to analyze due to their inhomogeneous nature. In order to use composite material systems safely and efficiently, researchers have studied and developed the underlying theory to explain their structural response and failure mechanisms under loading.

Composite structures, or parts, are created by placing layers of material onto one another to create a laminate. The number of individual layers in a laminate, along with their material and orientation, determine the constitutive response of the laminate as a whole. This gives a laminate that behaves in an orthotropic fashion and differentiates composite laminates from other commonly used isotropic materials. An orthotropic material does not to have the same material properties in any arbitrarily chosen direction. Since a laminate is composed of a number of discrete layers with varying directional properties, there is an inherent material property dependence based on the coordinate system chosen. The application of Classical Lamination Theory (CLT) can help predict the net global material behavior of a laminate based on the individual plies in the laminate. Similarly oriented plies can be thought of as having their own material properties based on an individual material coordinate system. This fact is used to determine the constitutive response of the entire laminate when loaded. 
CLT itself, however, cannot be used to analyze and design composite structures for things like strength, impact resistance, or fatigue. These design considerations require an understanding of the complicated and coupled failure modes of a composite laminate. Idealizations in deriving CLT, such as perfect bonding between plies and negligible edge effects, mean that it cannot be used to predict failure modes such as separation or delamination between plies. While this is not the only possible failure mode of a composite laminate, it is one that is important to understand if a structure may be exposed to dynamic loading. Ply delamination can reduce strength, stiffness, and the resistance to buckling of a structure. Figure 1 shows an example of a delamination in a laminated composite.

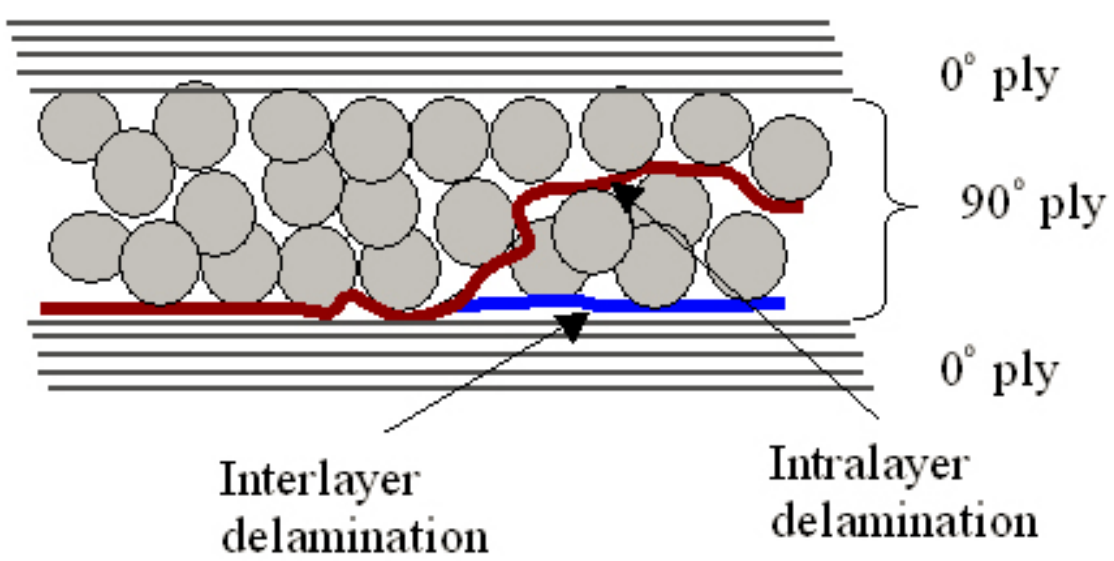

Figure 1 Example of delamination in a laminated composite [1]

An interlaminar delamination is characterized by a separation failure between two adjacent plies. A second delamination failure mode, called an intralaminar delamination, may also occur when failure occurs within a ply. In most cases, the term "delamination" refers to interlaminar failure while intralaminar crack growth falls under the general term 
of "matrix cracking." Both failure modes are the result of defects in the bond between lamina in the laminate (or constituents in the composite itself) and can ultimately result in complete failure of a structure. Two common examples where delamination should be carefully considered are airplane wings and bicycle frames. In both of these cases, a delamination may be induced by a simple tool drop or rock impact and a structural failure may lead to serious safety issues.

\subsection{Classical Lamination Theory}

Classical Lamination Theory (CLT) is the theoretical foundation for analyzing and designing composite structures. A brief discussion of CLT is given herein as presented in detail by Agarwal [2]. Generalized Hooke's law relates stress to strain for a general state of stress for a body and is given in indicial notation as

$$
\sigma_{i j}=E_{i j k l} \varepsilon_{i j},
$$

where $E_{i j k l}$ is the fourth order elastic tensor. In general, this tensor can have 81 elastic constants but can be reduced to have nine nonzero constants for an orthotropic material [3]. For an orthotropic material, the elastic tensor can be written as

$$
E_{i j k l}=\left[\begin{array}{cccccc}
E_{1111} & E_{1122} & E_{1133} & 0 & 0 & 0 \\
E_{1122} & E_{2222} & E_{2233} & 0 & 0 & 0 \\
E_{1133} & E_{2233} & E_{3333} & 0 & 0 & 0 \\
0 & 0 & 0 & E_{1111} & 0 & 0 \\
0 & 0 & 0 & 0 & E_{1111} & 0 \\
0 & 0 & 0 & 0 & 0 & E_{1111}
\end{array}\right] .
$$

Since four indices are not necessary to describe Hooke's law for an orthotropic material, contracted (or Voigt) notation is typically used giving 


$$
\sigma_{i}=C_{i j} \varepsilon_{j}, \quad i, j=1,2,3,4,5,6
$$

as the stress-strain relationship in indicial form and in matrix form in (4).

$$
\left\{\begin{array}{c}
\sigma_{1} \\
\sigma_{2} \\
\sigma_{3} \\
\tau_{23} \\
\tau_{13} \\
\tau_{12}
\end{array}\right\}=\left[\begin{array}{cccccc}
C_{11} & C_{12} & C_{13} & 0 & 0 & 0 \\
C_{12} & C_{22} & C_{23} & 0 & 0 & 0 \\
C_{13} & C_{23} & C_{33} & 0 & 0 & 0 \\
0 & 0 & 0 & C_{44} & 0 & 0 \\
0 & 0 & 0 & 0 & C_{55} & 0 \\
0 & 0 & 0 & 0 & 0 & C_{66}
\end{array}\right]\left\{\begin{array}{c}
\varepsilon_{1} \\
\varepsilon_{2} \\
\varepsilon_{3} \\
\gamma_{23} \\
\gamma_{13} \\
\gamma_{12}
\end{array}\right\} .
$$

It is important to note that the constants in the stiffness matrix $C_{i j}$ are given such that the engineering shearing strains are used, rather than the tensorial shearing strains in the $\varepsilon_{i j}$ matrix given in Eq. (1). Eq. (4) gives a three-dimensional stress-strain relationship for an orthotropic material with the reference axes aligned with the material axes. This is known as a relationship for a specially orthotropic material and can be transformed into a relationship for a generally orthotropic material using transformation matrices. Since symmetry will not necessarily exist in this transformed state, the stiffness matrix for a generally orthotropic material will likely be fully populated and can be found using the nine independent constants in the specially orthotropic stiffness matrix [2].

Many times in structural applications, it is convenient to assume a state of plane stress. A composite that is only loaded in the plane of the laminate can be assumed to have the stress-strain response shown in Eq. (5) below.

$$
\left\{\begin{array}{c}
\sigma_{1} \\
\sigma_{2} \\
\tau_{12}
\end{array}\right\}=\left[\begin{array}{ccc}
Q_{11} & Q_{12} & 0 \\
Q_{12} & Q_{22} & 0 \\
0 & 0 & Q_{66}
\end{array}\right]\left\{\begin{array}{c}
\varepsilon_{1} \\
\varepsilon_{2} \\
\gamma_{12}
\end{array}\right\} .
$$


The $[Q]$ matrix represents the stiffness matrix for plane stress in material coordinates, which is useful for a single lamina, or ply. Through tensor transformation of each lamina and the integration of each lamina through the thickness of the laminate using plate theory, a complete constitutive equation can be written. This equation, given in Eq. (6), relates resultant extensional/shearing forces and bending moments to strain and curvatures in the laminate.

$$
\left\{\begin{array}{c}
N_{x} \\
N_{y} \\
N_{x y} \\
M_{x} \\
M_{y} \\
M_{x y}
\end{array}\right\}=\left[\begin{array}{ccc}
A_{i j} & \vdots & B_{i j} \\
\cdots & \vdots & \cdots \\
B_{i j} & \vdots & D_{i j}
\end{array}\right]\left\{\begin{array}{c}
\varepsilon_{x} \\
\varepsilon_{y} \\
\gamma_{x y} \\
\kappa_{x} \\
\kappa_{y} \\
\kappa_{x y}
\end{array}\right\},
$$

where

$$
\begin{gathered}
A_{i j}=\sum_{k=1}^{N}\left(\overline{Q_{\imath \jmath}}\right)\left(z_{k}-z_{k-1}\right), \\
B_{i j}=\sum_{k=1}^{N}\left(\overline{Q_{l \jmath}}\right)\left(z_{k}{ }^{2}-z_{k-1}{ }^{2}\right), \\
D_{i j}=\sum_{k=1}^{N}\left(\overline{Q_{l \jmath}}\right)\left(z_{k}{ }^{3}-z_{k-1}{ }^{3}\right),
\end{gathered}
$$

and $k$ is the layer number, $N$ is the total number of layers and $\overline{Q_{l \jmath}}$ is the transformed stiffness matrix, and $z$ is the distance from the mid-plane of the laminate to the layer of interest. The significance of the above equations in low velocity impact (which inherently will result in a three-dimensional state of stress) is typically not considered. However, since the equations of CLT are computationally efficient to solve, it is advantageous to 
use this theory to examine the resistance to delamination of a laminate, as was shown by Liu [4].

\subsection{Literature Review}

\subsubsection{Low Velocity Impact of Composites}

Many researchers have studied low velocity impact of carbon fiber reinforced structures and the topic is discussed in many books in the field of composites. The literature on low velocity impact shows that low velocity impact can result in both delamination between plies and the onset of matrix cracking $[5,6,7,8,9]$. Liu discusses an analytical method based on the bending stiffness of composite sublaminates computed using CLT to predict the relative size and shape of a delamination $[4,10]$. While CLT is derived considering a plane stress condition, Liu proposes that bending stiffness plays the largest role in the propagation of a delamination under low velocity impact. Since low velocity impact does not produce appreciable dynamic membrane effects in most composite material systems, the impact can be treated as global bending in the laminate. Other researchers have also performed experiments to compare quasi-static testing to dynamic impact testing to test this hypothesis [11].The extent of damage due to low velocity impact is largely dependent on the laminate thickness, ply orientations, impact energy, impactor geometry, and resin system properties [10, 5].

\subsubsection{Bending Stiffness Mismatch}

Liu's bending stiffness mismatch method to predict the shape and relative size of a delamination in a composite plate under low velocity impact is based on the bending stiffness predicted by classical lamination theory [4]. Since the $\mathrm{D}_{11}$ and $\mathrm{D}_{22}$ terms determine the constitutive response relating the bending moments about an axis with the 
curvature deformations about that axis in a laminate, these terms represent a laminate's flexural stiffness. Using these terms, Liu defined a "bending stiffness mismatch" parameter, $M_{b}$, which is defined as

$$
M_{b}=\frac{D_{i j}\left(\theta_{b}\right)-D_{i j}\left(\theta_{t}\right)}{D_{i j}\left(0^{\circ}\right)-D_{i j}\left(90^{\circ}\right)} .
$$

This definition of $M_{b}$ normalizes the bending stiffness mismatch parameter with a cross-ply laminate of $0^{\circ}$ and $90^{\circ}$ sublaminate orientations since this is the largest possible bending stiffness mismatch. The $D_{i j}\left(\theta_{b}\right)$ term is the bottom sublaminate's flexural bending stiffness, $D_{11}$. Similarly, $D_{i j}\left(\theta_{t}\right)$ is the top sublaminate's flexural stiffness. This results in $M_{b}$ having a maximum magnitude of unity and a minimum magnitude of zero for an entirely unidirectional laminate. Assuming the top sublaminate and bottom sublaminate material directions are oriented at $90^{\circ}$ angles from each other, the equation for the bending stiffness mismatch of the laminate can be reduced to

$$
M_{b}=\cos (2 \theta) .
$$

Plotting Eq. 10 as a function in polar coordinates, where theta is the angle of interest and $M$ is the bending stiffness mismatch, creates a polar rose with four petals as shown in Figure 2. This graph can be used to approximate the "peanut" shape of the delamination that will occur between the two sublaminates in question. 


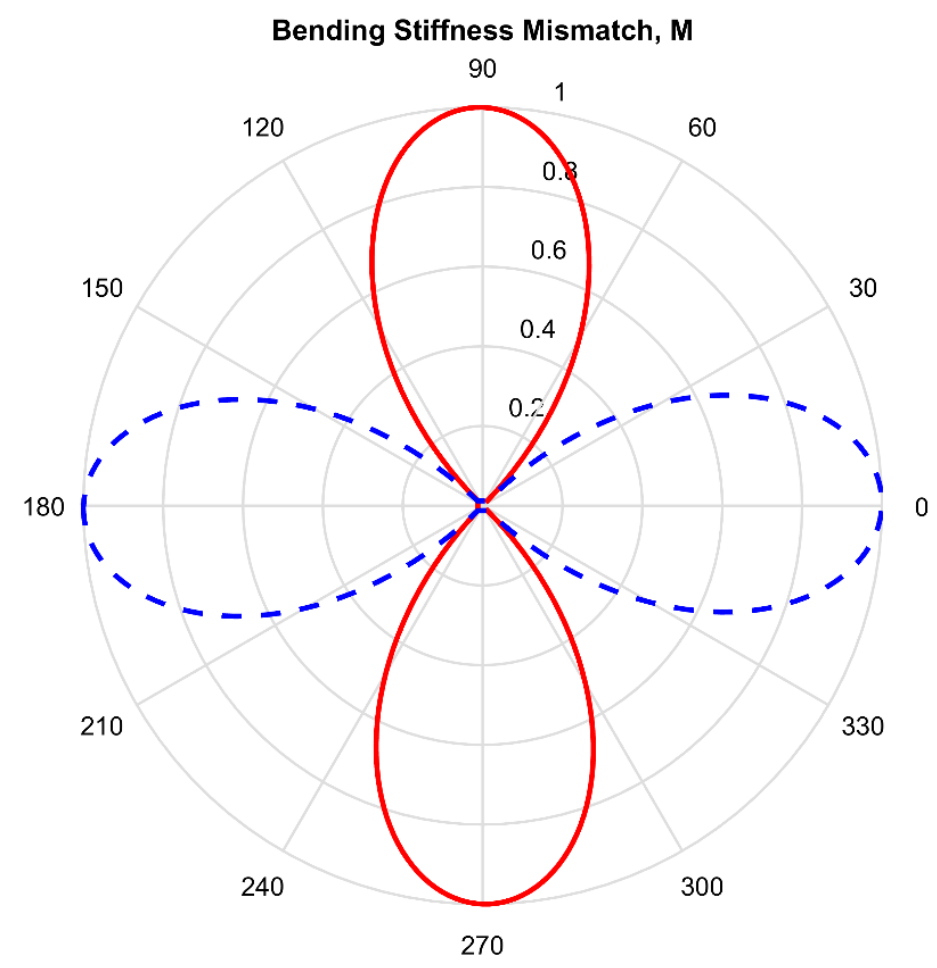

Figure 2 Bending stiffness mismatch plot

The delamination shape in Figure 2 is predicted for a $\left[0_{a} / 90_{a}\right]$ composite layup where $a$ is an arbitrary number of plies in the sublaminate. The red portion of the polar rose shown in Figure 2 signifies the locations where the bending stiffness mistmatch, $M$, is predicted to be positive, while the blue dotted portion depicts where it is negative. Based on this definition and the predicted concavity of the deflected laminate, it is concluded that a negative bending stiffness mismatch location will not result in a physical delamination [4]. If the orientation of the bottom ply (opposite to the ply where the impact directly occurs) is varied, the major axis of the peanut-shaped delamination is predicted to closely align with the orientation of the bottom ply [4]. This is depicted in Figure 3 . 


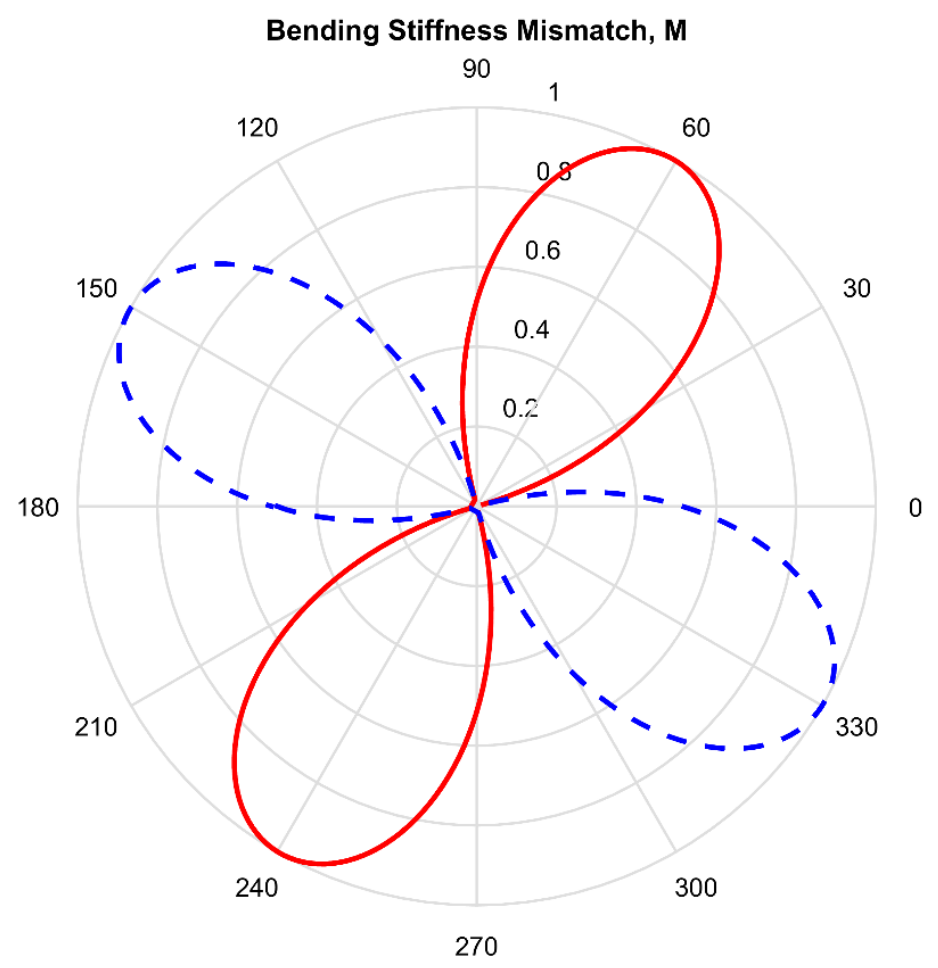

Figure 3 Back ply orientation effect on predicted delamination for $\left[-30_{\alpha} / 60_{\alpha}\right]$ laminate

\subsubsection{Fracture Experiments}

Since the delamination of a laminate is strongly correlated with the properties of the resin that is used, experimental methods are commonly used to predict the fracture toughness of a resin system. One energy-based method utilizes the strain energy release rate, $G_{I}$, to determine the energy required to fracture the matrix material in a composite laminate [12]. However, since a fracture delamination can be caused by multiple failure mechanisms, a strain energy release rate must be determined for each of these failure mechanisms. These modes are commonly referred to as Mode I, Mode II, and Mode III. Mode I is characterized by a failure due to tensile loading normal to the plane in which the fracture occurs, while Model II and Mode III fractures result from shear loading in the 
plane of the fracture where propagation of interlaminar defects may occur as shown in Figure 4.

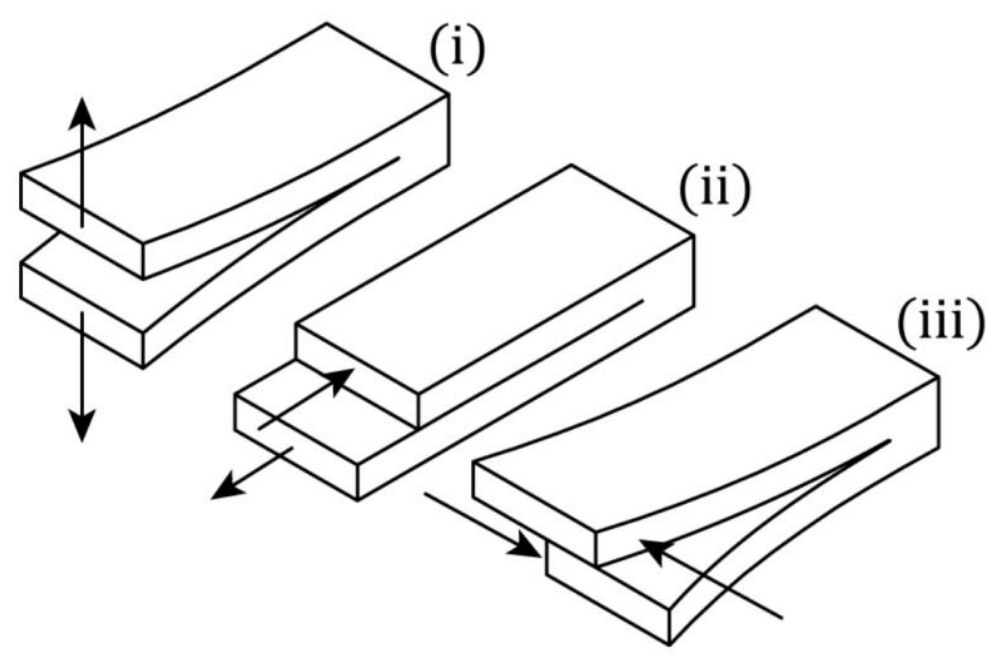

Figure 4 Modes I - III of matrix fracture [12]

A laminate subjected to practical loading conditions will likely experience failure due to a combination of these modes, making it difficult to precisely determine the damage evolution that leads to failure. At the micromechical level, this is a complex problem and taking a more macroscopic view can cause a loss in modeling fidelity that leaves a gap in understanding the true mechanisms of failure. The coupling of the three delamination fracture modes is commonly referred to as mixed-mode fracture since a combination of each mode of failure is present.

A double cantilever beam (DCB) experiment is commonly used to determine the Mode I fracture energy of composite material systems. The American Society of Testing and Materials (ASTM) has published testing guidelines in specification ASTM D5528-13 that outlines the procedure to determine the Mode I interlaminar fracture toughness of unidirectional fiber-reinforced polymer matrix composites [13]. Mode II and mixed 
Mode I/Mode II delamination fracture energies can be found using ASTM

D7905/D7905M-14 and ASTM D6671/D6671M-13e1, respectively [14, 15].

\subsubsection{Delamination Modeling Strategies}

Given the important role that delamination damage plays in the strength of a composite structure, many researchers have sought techniques to model delamination between composite laminae $[16,6,17,18,19,7,20,8,9,1]$. Cohesive element formulations have been developed attempting to accurately model the delamination failure mode and how it propagates. LS-DYNA ${ }^{\circledR}$, s implementation of these elements is discussed in Chapter 2. Cohesive elements have a constitutive response defined by a traction force and crack opening distance, unlike common element formulations with material constitutions governed by stress and strain [19]. Once a specified peak traction force is reached, damage occurs as a stiffness degradation of the cohesive element. Current research has focused on modeling strategies which model each ply or sublaminate as separate parts and create a bond between each respective part using cohesive elements (of finite or zero thickness). Since modeling every ply in a laminate, as well as creating cohesive elements between each ply, is too computationally costly, this technique requires knowledge of what ply interface the delamination failure may occur.

Other delamination models can predict the onset of delamination failure but cannot capture the progression of the delamination crack front. LS-DYNA ${ }^{\circledR}$ contains quadratic stress-based composite material failure models in *MAT_22,*MAT_054-55, and *MAT_59 [21]. As an optional add-on material model in LS-DYNA ${ }^{\circledR}, *$ MAT_162 was developed by Materials Sciences Corporation and is described as a progressive 
composite damage model. Researchers have used this proprietary model to predict the onset and propagation of delamination [22].

LS-DYNA $^{\circledR}$ also contains an additional method for delamination modeling known as a contact tiebreak definition. This method is based on the underlying cohesive element theory. However, unlike cohesive element formulations, it is not necessary to create a cohesive element mesh. As explained further in Section 2.2.2, composite ply segments are tied together and crack opening between them is governed by the theory and fracture parameters given by cohesive element formulations.

The virtual crack closure technique was developed to model delamination and, as its name suggests, is based on the strain energy released to open or close a crack. This fracture mechanics based approach was not used in this research but was recently implemented in the ABAQUS ${ }^{\circledR}$ finite element code [23].

\subsection{Thesis Goals}

In order to design structures that may be subject to low velocity impact quickly and efficiently, it is desirable to use an efficient theoretical tool, such as CLT, to predict how well a design will hold up. While CLT is limited to establishing the in-plane constitutive response of a laminate, Liu's suggestion that the bending stiffness mismatch parameter of a particular laminate is indicative of its resistance to delamination is one factor to consider in design for low velocity impact. Despite the fact that the interaction of failure modes in a composite laminate is complex, a high-level consideration of the relationship between bending stiffness mismatch and delamination resistance can be used to inform composite laminate design. Through the use of an LS-DYNA ${ }^{\circledR}$ finite element model and the analysis of accompanying experimental data, the ability of bending 
stiffness based methods to predict delamination during composite low-velocity impact events are analyzed. Impact testing on a simple laminate and nondestructive vibrothermography experiments are performed to compare the predictions of finite element modeling of delamination using a contact tiebreak definition. 


\section{CHAPTER 2: FINITE ELEMENT MODELING}

\subsection{Governing Equations}

While CLT is useful for analyzing thin composite parts under a specific set of assumptions, the finite element method provides a tool for a more rigorous analysis when necessary. The finite element method is implemented by discretizing a body into small parts, or elements. Each element consists of nodes with displacement and possibly rotational degrees of freedom. Between element nodes, shape functions are used as an assumed displacement field. In the simplest case, applied loads and known displacements are enforced as boundary conditions in the matrix equation

$$
\{F\}=[K]\{d\} .
$$

By inverting the global system stiffness matrix $[K]$, the unknown nodal degrees of freedom (displacements or rotations) in column vector $\{d\}$ may be computed. With $\{d\}$ known, unknown internal forces and external reaction forces are determined in column vector $\{F\}$. This approach is called an implicit scheme because solving for the displacement column vector $\{d\}$ requires the inversion of matrix $[K]$.

The method above is used to solve linear static problems. For dynamic problems, equilibrium is governed by the discretized force balance equation (in its simplest form) given by

$$
[M] \ddot{x}^{i}+[C] \dot{x}^{i}+[K] x^{i}=R^{i},
$$

where each dot signifies a time derivative of nodal displacement and the superscript $i$ represents the $i^{t h}$ time step. Matrix $[M]$ is the system mass matrix, $[C]$ is the system 
damping coefficient matrix, $[K]$ is the system stiffness matrix, and $R$ is a column vector of applied external loads.

Both implicit and explicit numerical methods for solving these equations are used. Implicit methods generally have superior stability properties but can be computationally costly since due to the inversion of large matrices in the problem's solution. However, if an alternate time discretization scheme is chosen, the same equations of motion can be solved using an explicit method. This generally requires lumped (diagonal) mass and damping coefficient matrices to avoid matrix inversion. LS-DYNA ${ }^{\circledR}$ utilizes a central difference time discretization scheme to numerically integrate the equations of motion in time [24]. As a result of choosing an explicit scheme, the time step taken by LS-DYNA ${ }^{\circledR}$ is restricted to ensure that stability of the numerical solution is preserved. The minimum time step is computed by LS-DYNA ${ }^{\circledR}$ and can be the limiting factor in how detailed of an analysis may be run. The time step must satisfy the Courant stability criterion in order for the deformation wave to be captured across the structure without skipping elements. For example, extremely small and stiff elements can result in very small time steps which makes the time of solution prohibitively long. Since impact events occur over small time periods, explicit analyses are typically utilized since the computational cost of matrix inversion in an implicit method may be too costly.

\subsection{Mode I Delamination: Double Cantilever Beam}

In order to validate the contact tiebreak delamination model in LS-DYNA ${ }^{\circledR}$ for use in a low velocity impact simulation, a simple Mode I model was developed. This model was made very similar to a study described in a report for Lawrence Livermore National Laboratory (LLNL) by Kay [19] in order to verify the contact tiebreak model 
was working as expected. While Kay's report meshed and defined cohesive elements, the contact tiebreak definition in LS-DYNA ${ }^{\circledR}$ use very similar parameters. The model also closely aligns with ASTM D5528-13 for determining the Mode I interlaminar fracture toughness of unidirectional fiber-reinforced polymer matrix composites [13].

\subsubsection{Mode I Simulation Geometry}

Figure 5 shows the geometry for a finite element simulation of Mode I fracture using a double cantilever beam test. This based on a 24-ply unidirectional carbon fiber laminate. The top and bottom sublaminates are each 0.0935 inches thick for a total laminate thickness of 0.187 inches. The length and width of the beam are 4.921 inches and 0.945 inches, respectively. The specimen was assumed to be pre-cracked for the first 1.5 inches on the end where the prescribed displacement was applied as shown in Figure 5.

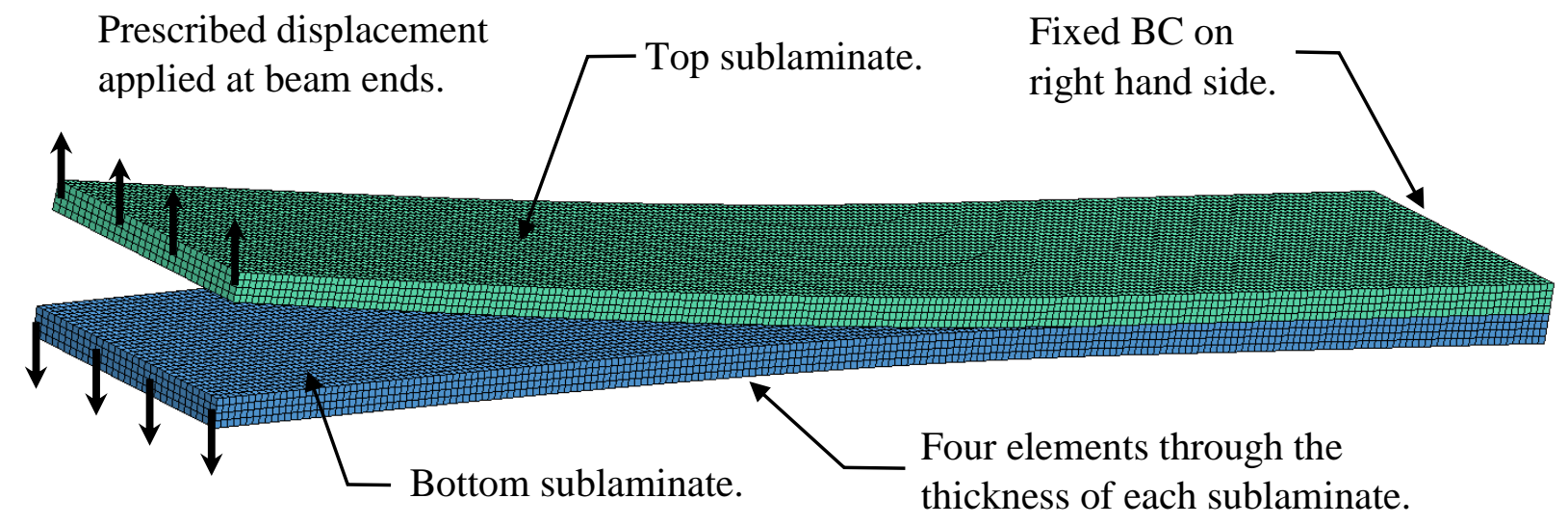

Figure 5 LS-DYNA ${ }^{\circledR}$ Mode I DCB FE simulation geometry and mesh 


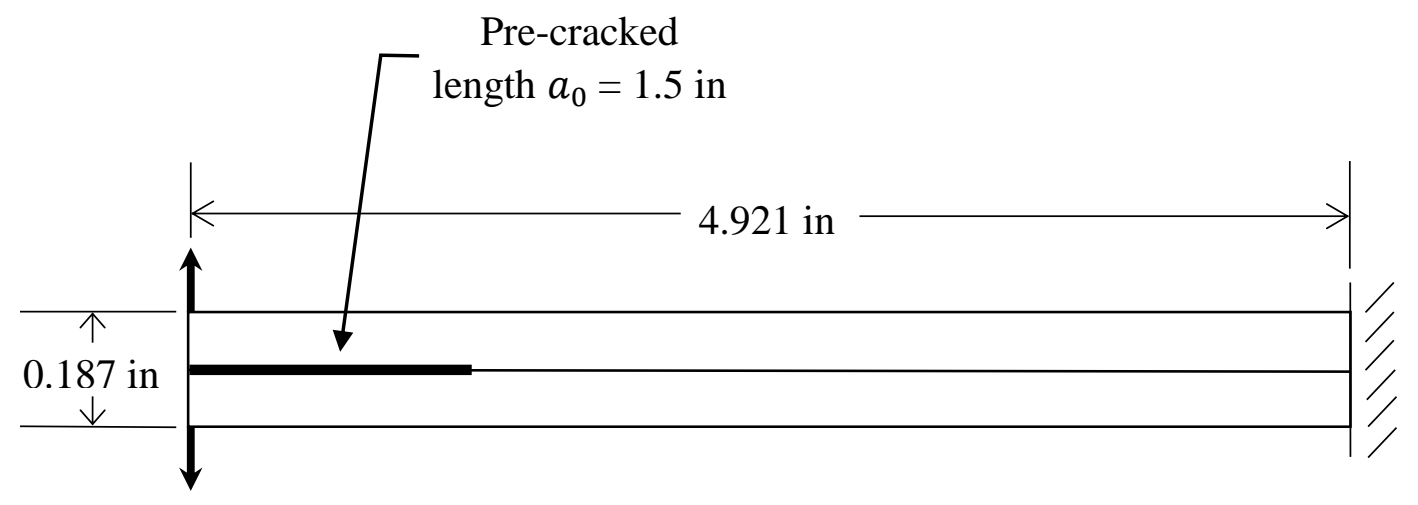

Figure 6 Pre-cracked length for Mode I DCB simulation

\subsubsection{Contact Tiebreak Used in Mode I Simulation}

As mentioned previously, there are a few different options to model the delamination failure mode in a composite structure. Since both delamination initiation and propagation were desired, composite material models in LS-DYNA ${ }^{\circledR}$ that utilize delamination initiation failure criteria were not employed. These failure models include *MAT_22, *MAT_054-55, and *MAT_59, which have quadratic stress-based failure criteria as mentioned in Section 1.2.4. Instead, a contact tiebreak definition was used to define contact between the two sublaminates in the composite panel. This contact tiebreak definition is based on the theoretical formulation of cohesive elements. However, rather than creating and meshing cohesive elements between plies where delamination may occur, segments on the bottom of an upper sublaminate and on the top of a lower adjacent sublaminate are "tied" together at the beginning of the analysis. As the finite element numerical solution evolves in time, a check is performed between each segment to determine whether delamination has occurred between the elements in the upper and lower sublaminates [25]. 
Specifically, contact tiebreak definition *CONTACT_AUTOMATIC_ONE_ WAY_SURFACE_TO_SURFACE_TIEBREAK with OPTION 9 was utilized in the Mode I DCB simulations as well as the laminated composite panel impact simulations. As mentioned previously, this contact formulation is based on the cohesive element formulation and constitutive response in *MAT_138 in LS-DYNA ${ }^{\circledR}$. Since the contact tiebreak definition is so closely related to the cohesive formulation of Material 138 in LSDYNA $^{\circledR}$, it is useful to discuss the underlying theory here. Material 138 is a mixed-mode cohesive element formulation that takes into account the interaction between Mode I (pure normal traction) and Mode II (pure shear traction) delamination. The tractionseparation law for this material is given by linear softening once peak traction stress has been reached and is shown in Figure 7. The variables specified in the input deck for the contact tiebreak definition that relate to Material 138 are given in Table 1 [21].

Assuming a bilinear traction-separation law results in a simple relationship between the energy release rate, peak traction stresses, and ultimate displacement between plies when failure occurs. QMAX in Figure 7 represents the ultimate displacement in either the normal or the tangential direction (UND or UTD, respectively) before failure of the contact tiebreak is observed. The ultimate normal and tangential displacements are calculated from the specified peak tractions (NFLS and SFLS) given in Table 1, as well as the specified energy release rates (GIC and GIIC) using Eq. (14) and Eq. (15) below

$$
G_{I C}=T x \frac{U N D}{2},
$$




$$
G_{I I C}=S x \frac{U T D}{2}
$$

However, since there may be both normal and tangential relative motion between plies or sublaminates, a parameter $\delta_{m}=\sqrt{\delta_{I}^{2}+\delta_{I I}^{2}}$, where $\delta_{I}=\delta_{3}$ (see Figure 8) is the separation in the normal direction (mode I) and $\delta_{I I}=\sqrt{\delta_{1}^{2}+\delta_{2}^{2}}$ is the resultant separation in the tangential plane, is defined. The parameter $\delta_{m}$ is then compared to the mixed-mode damage initiation

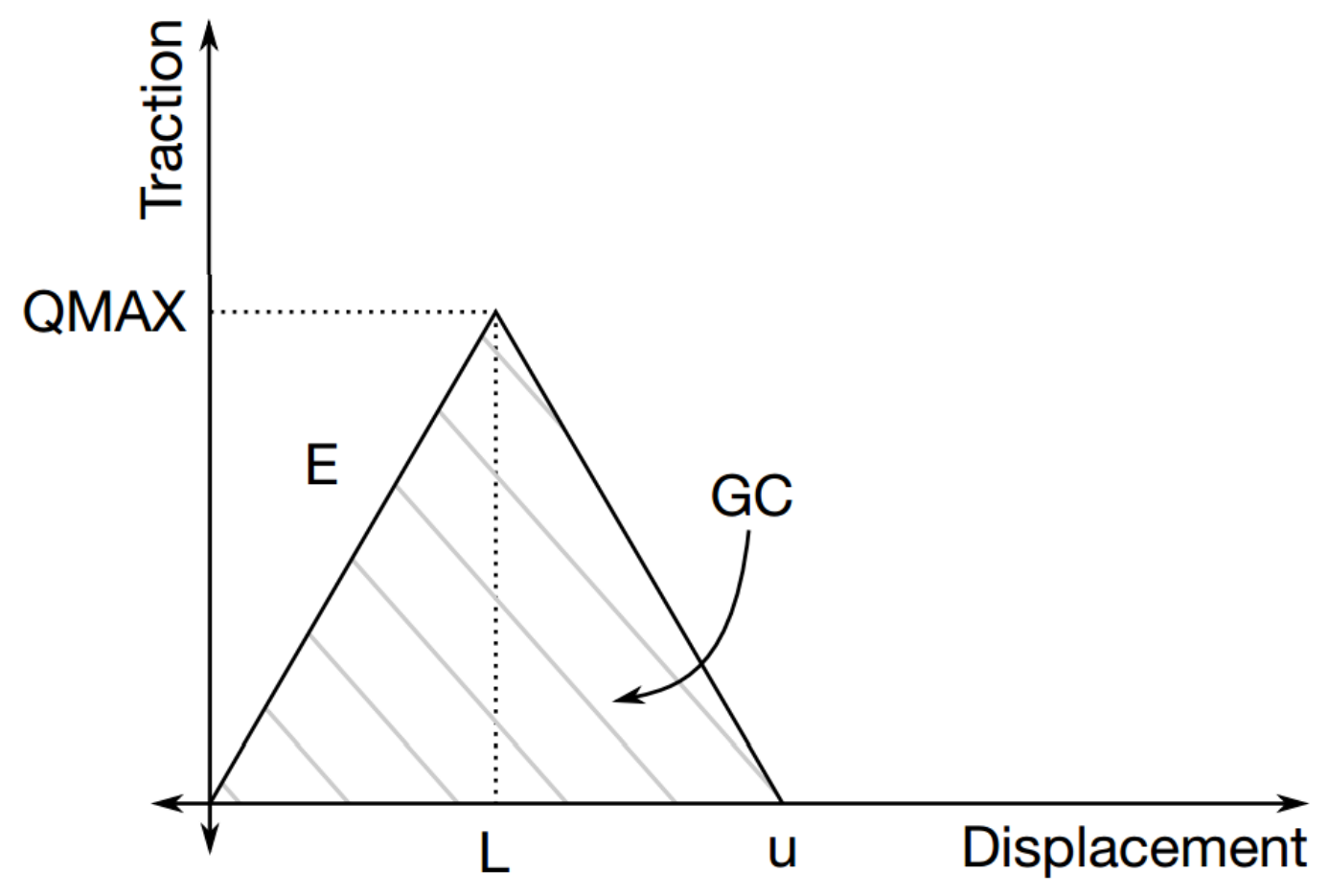

Figure 7 Bilinear traction-separation law for *MAT_138 
Table 1 LS-DYNA® Keyword variables of interest [25]

\begin{tabular}{|c|c|l|c|}
\hline $\begin{array}{c}\text { Contact } \\
\text { Tiebreak }\end{array}$ & Material 138 & \multicolumn{1}{|c|}{ Description } & Units \\
\hline NFLS & T & Peak traction in normal direction & Stress \\
\hline SFLS & S & Peak traction in tangential direction & Stress \\
\hline PARAM & XMU & Exponent of mixed-mode criteria & Unitless \\
\hline ERATEN & GIC & Energy release rate for Mode I & Stress*length \\
\hline ERATES & GIIC & Energy release rate for Mode II & Stress*length \\
\hline CT2CN & ET/EN & $\begin{array}{l}\text { Ratio of tangential stiffness to normal } \\
\text { stiffness }\end{array}$ & Unitless \\
\hline CN & EN & Normal stiffness & Stress/length \\
\hline
\end{tabular}

displacement $\delta^{0}$. Once this value is exceeded, softening occurs. $\delta^{0}$ is given by

$$
\delta^{0}=\delta_{I}^{0} \delta_{I I}^{0} \sqrt{\frac{1+\beta^{2}}{\left(\delta_{I I}^{0}\right)^{2}+\left(\beta \delta_{I}^{0}\right)^{2}}}
$$

where $\delta_{I}^{0}=\frac{T}{E N}$ and $\delta_{I I}^{0}=\frac{S}{E T}$ are the single mode damage initiation separations and $\beta=\delta_{I I} / \delta_{I}$ is defined as the "mode mixity." The ultimate mixed-mode displacement $\delta^{F}$ for complete failure based on the power law is

$$
\delta^{F}=\frac{2\left(1+\beta^{2}\right)}{\delta^{0}}\left[\left(\frac{E N}{G I C}\right)^{X M U}+\left(\frac{E T x \beta^{2}}{G I I C}\right)^{X M U}\right]^{-\frac{1}{X M U}}
$$

Using the Benzeggagh-Kenane law,

$$
\delta^{F}=\frac{2}{\delta^{0}\left(\frac{1}{1+\beta^{2}} E N^{\gamma}+\frac{\beta^{2}}{1+\beta^{2}} E T^{\gamma}\right)^{\frac{1}{\gamma}}}\left[G I C+(G I I C-G I C)\left(\frac{\beta^{2} E T}{E N+\beta^{2} E T}\right)^{|X M U|}\right]
$$


The power law and Benzeggagh-Kenane law are empirical damage evolution functions. These functions are used to determine when complete failure of the contact tiebreak definition has occurred [26].

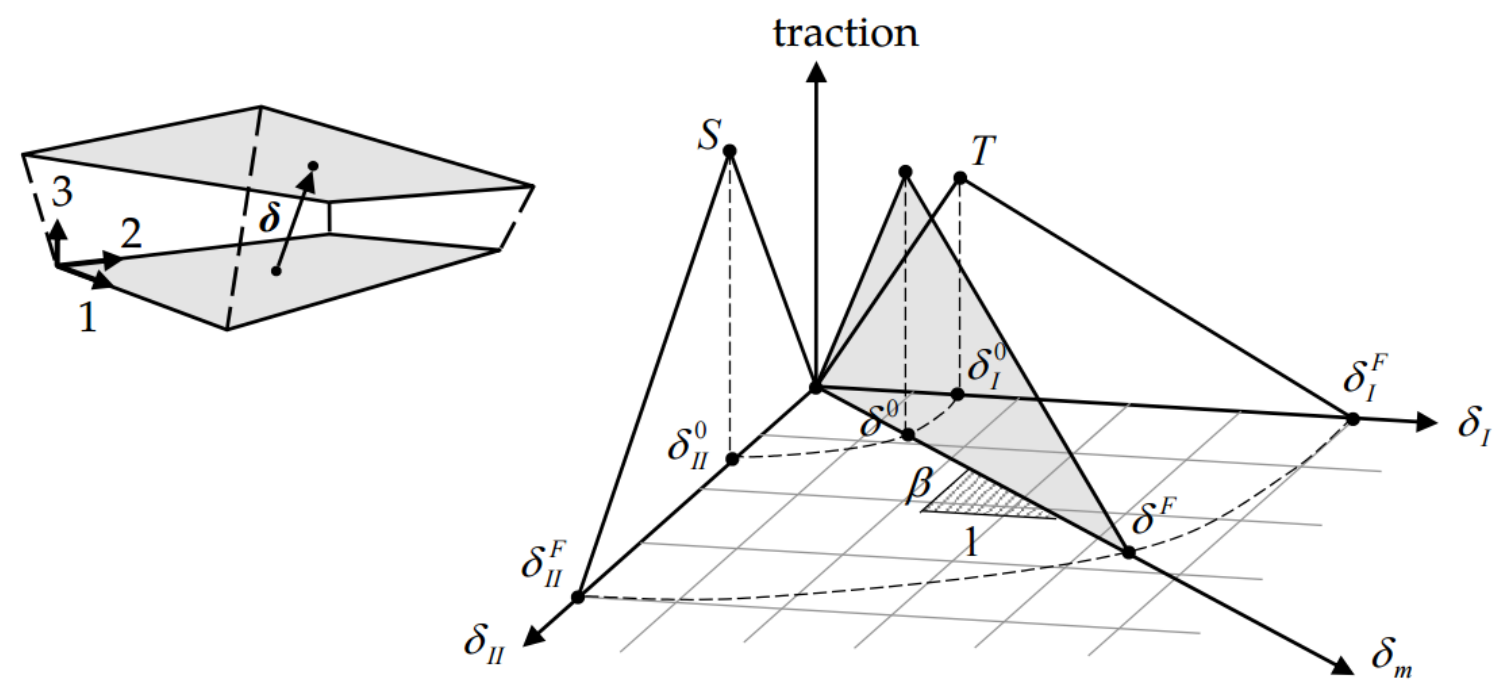

Figure 8 Mixed-mode traction-separation law

Figure 8 shows the interaction between Mode I and Mode II fracture in the tractionseparation law for *MAT_138.

\subsubsection{Finite Element Parameters}

Reduced LS-DYNA ${ }^{\circledR}$ keyword input files are included in Appendix C. This keyword file includes the necessary cards to run a similar finite element model. In order to reduce the length of the file, element and node definitions have been omitted.

\subsubsection{Mesh \& Contact Tiebreak Mesh Dependency}

In order to ensure that the bending deformations in the upper and lower sublaminates were accurately modeled, four elements were used through the thickness of each sublaminate in the final mesh. With an aspect ratio of about 16 (length to thickness) 
the deflection of the sublaminates opening prior to crack propagation are dominated by bending deformation. Because of this, it is necessary to use four elements through the thickness to avoid shear locking issues. Aspect ratios for each element in the mesh were kept to about 1.35 for high quality elements, with the larger element dimension in the sublaminate width direction.

Since the contact tiebreak definition is based on the interface between the upper and lower sublaminates, there is inherent mesh dependency in simulated delamination in this manner. It is not well established how to determine the number of elements required at the interlaminar interface to achieve the most reliable results but it is known that a finer mesh will behave more realistically. Because of this, a lower peak interface traction force is needed for coarser meshes [24]. A peak traction force of 4000 psi was determined to give steady crack growth for the mesh shown. Table 2 shows the final contact tiebreak properties used in the Mode I simulation.

One of the most difficult parameters to determine in this model was the value for the normal contact stiffness ( $\mathrm{CN}$ in Table 2$)$ between the contact tiebreak segments. A contact stiffness that was too large resulted in numerical instabilities and caused LSDYNA $^{\circledR}$ to terminate with an error. On the other hand, much lower contact stiffness values resulted in delamination propagation much faster than expected. Through many iterations and observations, the current value shown in Table 2 gave results that most resembled observed Mode I delamination testing. 
Table 2 Values used for contact tiebreak variables in Mode I simulation

\begin{tabular}{|c|c|c|}
\hline $\begin{array}{c}\text { Contact Tiebreak } \\
\text { Variable }\end{array}$ & Value & Units \\
\hline NFLS & 4.0 & $\mathrm{ksi}$ \\
\hline SFLS & 4.0 & $\mathrm{ksi}$ \\
\hline PARAM & 2.0 & $\mathrm{~N} / \mathrm{A}$ \\
\hline ERATEN & 0.7423 & $\mathrm{psi}-\mathrm{in}$ \\
\hline ERATES & 0.7423 & $\mathrm{psi}-\mathrm{in}$ \\
\hline CT2CN & 1.0 & $\mathrm{~N} / \mathrm{A}$ \\
\hline CN & $8.909 \mathrm{e} 7$ & $\mathrm{psi} / \mathrm{in}$ \\
\hline
\end{tabular}

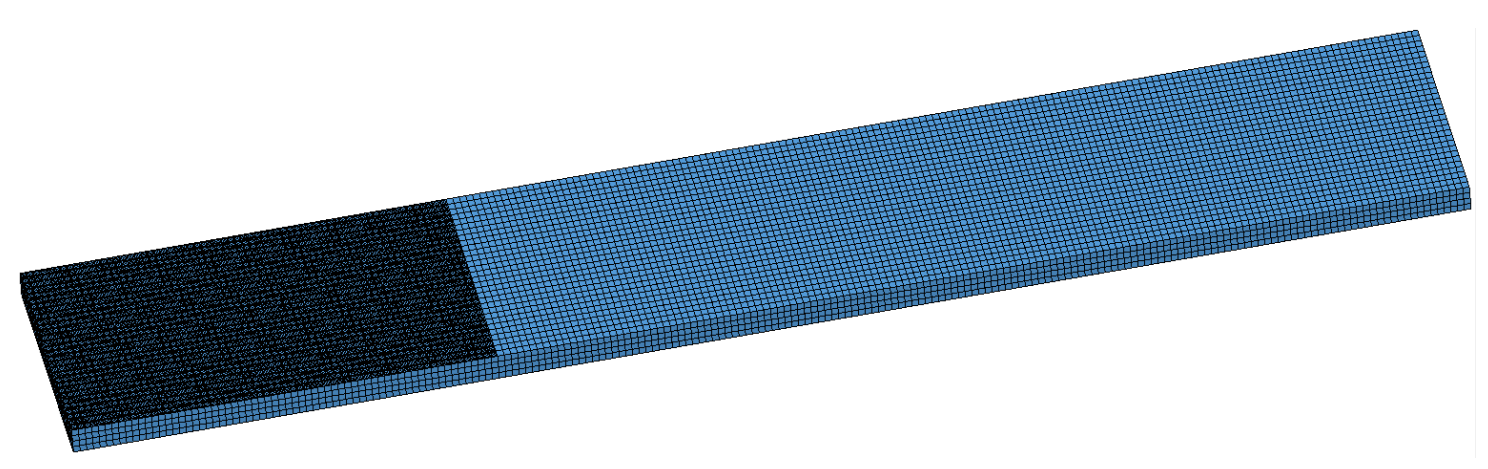

Figure 9 Bottom sublaminate pre-cracked segments with automatic contact

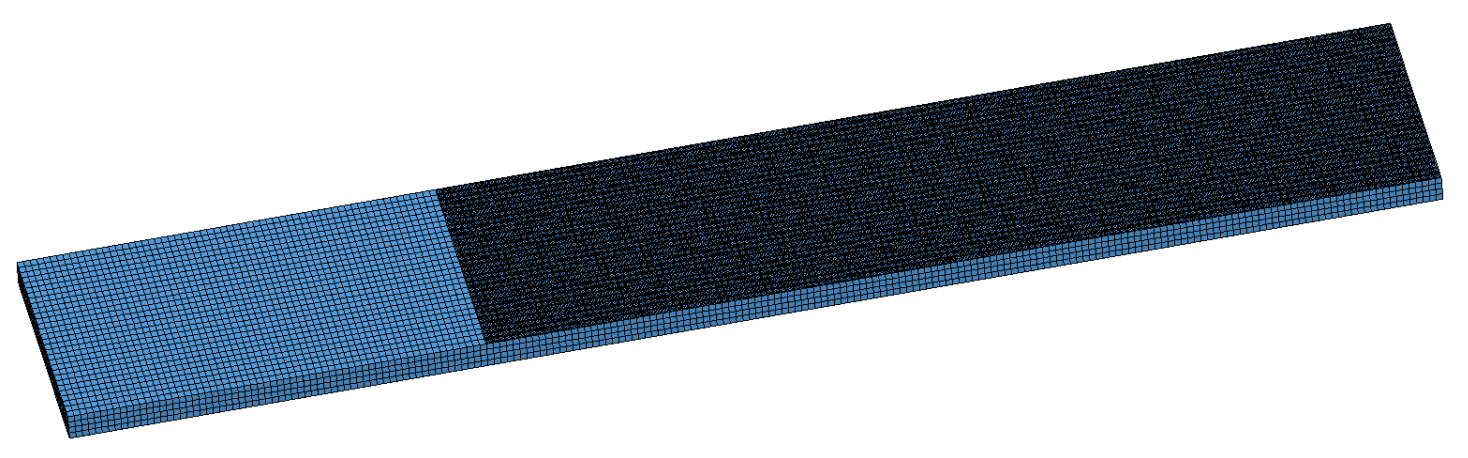

Figure 10 Bottom sublaminate contact tiebreak segments 
Figure 9 and Figure 10 depict the segments used for traditional surface-to-surface automatic contact and for the contact tiebreak definition. A segment in LS-DYNA ${ }^{\circledR}$ is defined as one of the faces on a 3-D solid element. Contact definitions between different portions of a model can be defined in multiple ways. For the contact tiebreak and automatic surface to surface contact definitions, contact segments were specified between segments on the top sublaminate bottom face and the bottom laminate upper face. The black segments shown in the bottom sublaminate in Figure 10 simulate the built in delamination portion of the top and bottom sublaminate interface at the mid-plane of the entire laminate. Section 2.2.1 defines this pre-cracked length as $a_{0}$. Similarly, the black colored segments in Figure 9 represent the location where the contact tiebreak definition is defined. It is in this region where the delamination will propagate as the load is increased and the peak traction values are exceeded between the upper and lower segments.

\subsubsection{Element Type}

Knowing that delamination modeling was the major objective of this research, solid elements were chosen since the an accurate consideration of delamination requires three-dimensional states of stress throughout the composite. Since delamination is considered at the interlaminar interface, normal and shearing stresses between the two laminae play a large role in the progression of a crack. Traditional plane-stress shell elements do not consider out-of-plane normal stresses, which delamination is dependent on [25]. Since an accurate initial beam stiffness prior to crack opening was desired, fully integrated three-dimensional solid elements with selective-reduced integration (solid element type-2 in LS-DYNA ${ }^{\circledR}$ ) were chosen. While computational efficiency is sacrificed 
for accuracy, this was determined to be an acceptable trade off given that accurate initial bending stiffness was desired. Selective reduced integration (through the use of the "Bbar" method in LS-DYNA ${ }^{\circledR}$ ) also helps control volumetric locking that may occur. Volumetric locking can occur when a material with a large value of Poisson's ratio exhibits incompressibility and is a negative result of come finite element formulations. The formulation is also well suited for problems utilizing elements with poor aspect ratio and have been introduced to avoid transverse shear locking [24]. Transvers shear locking can occur when a structure should experience bending loads but cannot carry the load in bending due to coarse element discretization through the thickness of the structure in bending. Rather than the top and bottom of the element experiencing normal strains and transverse deformation, the element may experience fictitious shearing strain to attain the same transverse deflection.

\subsubsection{Material Constitutive Response}

As mentioned in Section 1.2.4, a few different material models in LS-DYNA ${ }^{\circledR}$ are capable of modeling the behavior of orthotropic composite materials. In order to isolate the performance of the contact tiebreak definition, a basic orthotropic material was chosen. Material Type 2, *MAT_ORTHOTROPIC_ELASTIC, was used. Table 3 shows the material properties used for this material. As its name suggests, this material has an orthotropic constitutive response with entirely elastic behavior. Material properties from Kay's [19] report were used in order to validate contact tiebreak behavior in LS-DYNA ${ }^{\circledR}$ compared to cohesive elements. 
Table 3 Material properties for orthotropic Material Type 2 in LS-DYNA ${ }^{\circledR}$ [19]

\begin{tabular}{|c|c|c|}
\hline Property & Value & Units \\
\hline $\mathrm{E}_{\mathrm{a}}$ & 7.50 & Msi \\
\hline $\mathrm{E}_{\mathrm{b}}$ & 1.28 & Msi \\
\hline $\mathrm{E}_{\mathrm{c}}$ & 2.28 & Msi \\
\hline$v_{\mathrm{ba}}$ & 0.02545 & N/A \\
\hline$v_{\mathrm{ca}}$ & 0.02545 & N/A \\
\hline$v_{\mathrm{cb}}$ & 0.30000 & N/A \\
\hline $\mathrm{G}_{\mathrm{ab}}$ & 0.6601 & Msi \\
\hline $\mathrm{G}_{\mathrm{bc}}$ & 0.6601 & Msi \\
\hline $\mathrm{G}_{\mathrm{ca}}$ & 0.6601 & Msi \\
\hline$\rho$ & $1.45 \mathrm{E}-04$ & $\mathrm{lbf}-\mathrm{s}^{2} / \mathrm{in}$ \\
\hline
\end{tabular}

\subsubsection{Boundary Conditions for Mode I Analysis}

One end of the laminate was held fixed in all degrees of freedom while a prescribed displacement was applied to the other ends of the double cantilever beams, as shown in Figure 5. The prescribed displacement was applied at a rate of $10 \mathrm{in} / \mathrm{s}$. This was applied using a defined load curve with a displacement of 0.5 inches over 0.05 seconds. It was found that this loading rate limited dynamic effects while still allowing for a reasonable solution time.

\subsubsection{Mass and Stiffness Damping Considerations}

The Mode I fracture test should ideally be performed under quasi-static testing conditions. Using a dynamic explicit analysis with a much faster prescribed displacement than the physical experiment results in artificial dynamic effects. It was found in numerous simulations that the Mode I simulation required a small amount of damping to reduce dynamic fluctuations. To resolve this issue, mass damping was applied equal to 10 percent of the critical mass damping coefficient. The critical mass damping coefficient is given by the equation 


$$
c_{c r}=\frac{4 \pi}{T}
$$

where $T$ is the period of the mode targeted for damping [27]. Since the double cantilever beams each deflect independent of the each other prior to delamination propagation, the lowest fundamental frequency of a single cantilever beam with half the thickness of the laminate and a length of $a_{0}$ was used, as shown in Figure 11. To find this frequency, an

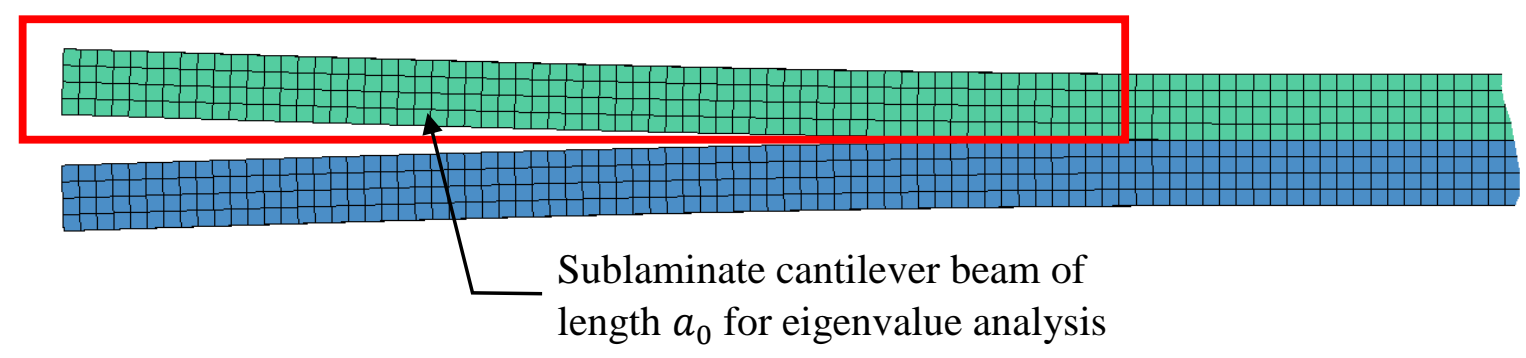

Figure 11 Portion of sublaminate used in modal analysis

implicit eigenvalue analysis was run and the lowest natural frequency was found to be 1517.54 Hz, with the first mode characterized by transverse bending shown in Figure 12. This was used to find the value of the critical damping coefficient of 1907 seconds. Figure 18 and Figure 19 in Section 2.2.4 show the difference in the force-displacement response with and without mass damping. The overall response before and after crack propagation is very similar in both cases. In the linear region, mass damping achieves its goal of damping out spurious oscillations prior to crack propagation. In addition, the effect of part stiffness damping was also explored with 10 percent of critical part stiffness damping. It was found that this had little effect on the resulting response. 


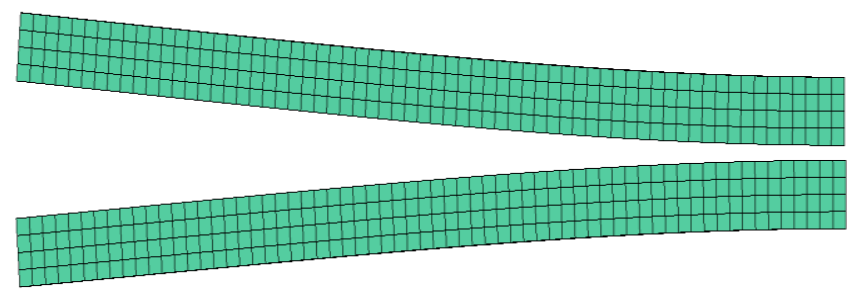

Figure 12 First natural frequency mode for the double cantilever beams in bending

\subsubsection{Results for Mode I Simulation}

If the pre-cracked portion of the beam truly exhibited a clamped boundary condition, the initial slope of the force vs. crack displacement curve would be the bending stiffness of the sublaminates prior to crack propagation. Since the pre-cracked length was 1.5 inches, each upper and lower sublaminate should deform with a transverse bending stiffness near that of a cantilever beam of the same length. Beam theory predicts that the bending stiffness of a cantilever beam is given by the expression

$$
k=\frac{3 E I}{L^{3}}
$$

Using the property given in Table 3 for $\mathrm{E}_{\mathrm{a}}$ and the width, thickness, and length of the precracked portion of the sublaminates given in Figure 5 and Figure 11 resulted in a bending stiffness of $429 \mathrm{lb}_{\mathrm{f}} /$ in. However, the lack of a true clamped boundary condition at the end of the delaminated region results in additional compliance. This is accounted for in the ASTM Mode I specification using a modified beam theory approach to determine the effective beam length when calculating the fracture energy [13]. 


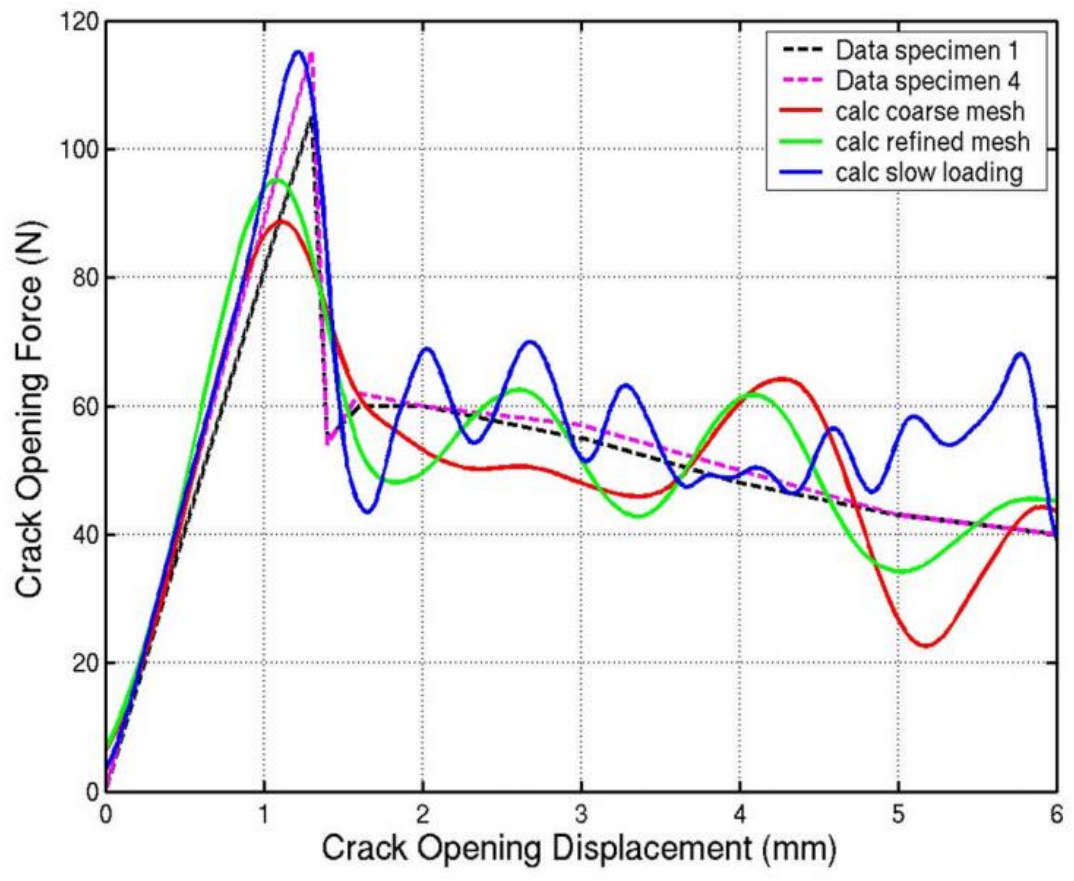

Figure 13 Force-displacement response for similar DCB simulation by Kay [19]

The response for the Double Cantilever Beam (DCB) simulation that this Mode I simulation in LS-DYNA ${ }^{\circledR}$ was based on is shown in Figure 13. While Kay’s study was done using metric units, the simulation in this research was performed using the English unit system. Kay's simulation resulted in an initial stiffness of about $457 \mathrm{lb}_{\mathrm{f}} / \mathrm{in}$. It was found that when using the contact tiebreak definition in LS-DYNA ${ }^{\circledR}$ to model the Mode I specimen, the pre-crack stiffness was about $344 \mathrm{lb}_{\mathrm{f}}$ /in and it shown in Figure 14. This $19.8 \%$ difference compared to beam theory can be attributed to the additional compliance at the root of the initial delamination. It was not clear how Kay was able to match the bending stiffness of the experimental data without accounting for the reduced compliance at the root of the crack. It may be the case that the geometry was adjusted to account for this difference since it was mentioned that the thickness of the simulation geometry was not exactly the same as the experimentally tested geometry. 


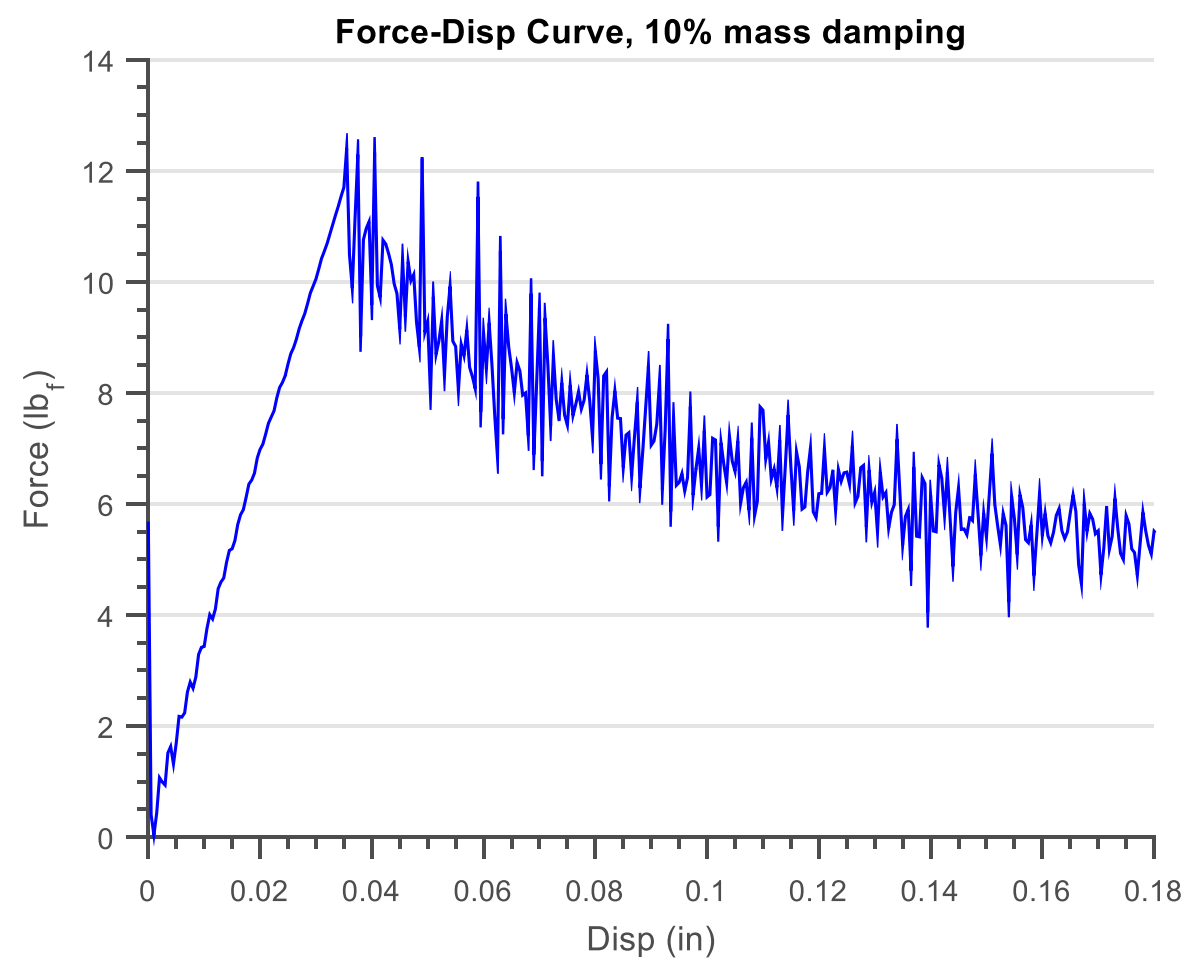

Figure 14 Crack opening force vs. crack opening distance for DCB specimen

Since it is not feasible to run a transient simulation with the prescribed displacement of a quasi-static experiment, a much faster loading rate was applied than suggested in ASTM-5528. Various loading rates were applied in an attempt to balance dynamic effects and computational efficiency. It was found that a loading rate of $10 \mathrm{in} / \mathrm{s}$ achieved a balance between these two variables. Figure 15 and Figure 16 depict the difference between two different loading rates. The slower loading rate of $5 \mathrm{in} / \mathrm{s}$ resulted in more high frequency oscillations during initial crack opening when compared with a faster loading rate of $10 \mathrm{in} / \mathrm{s}$. 


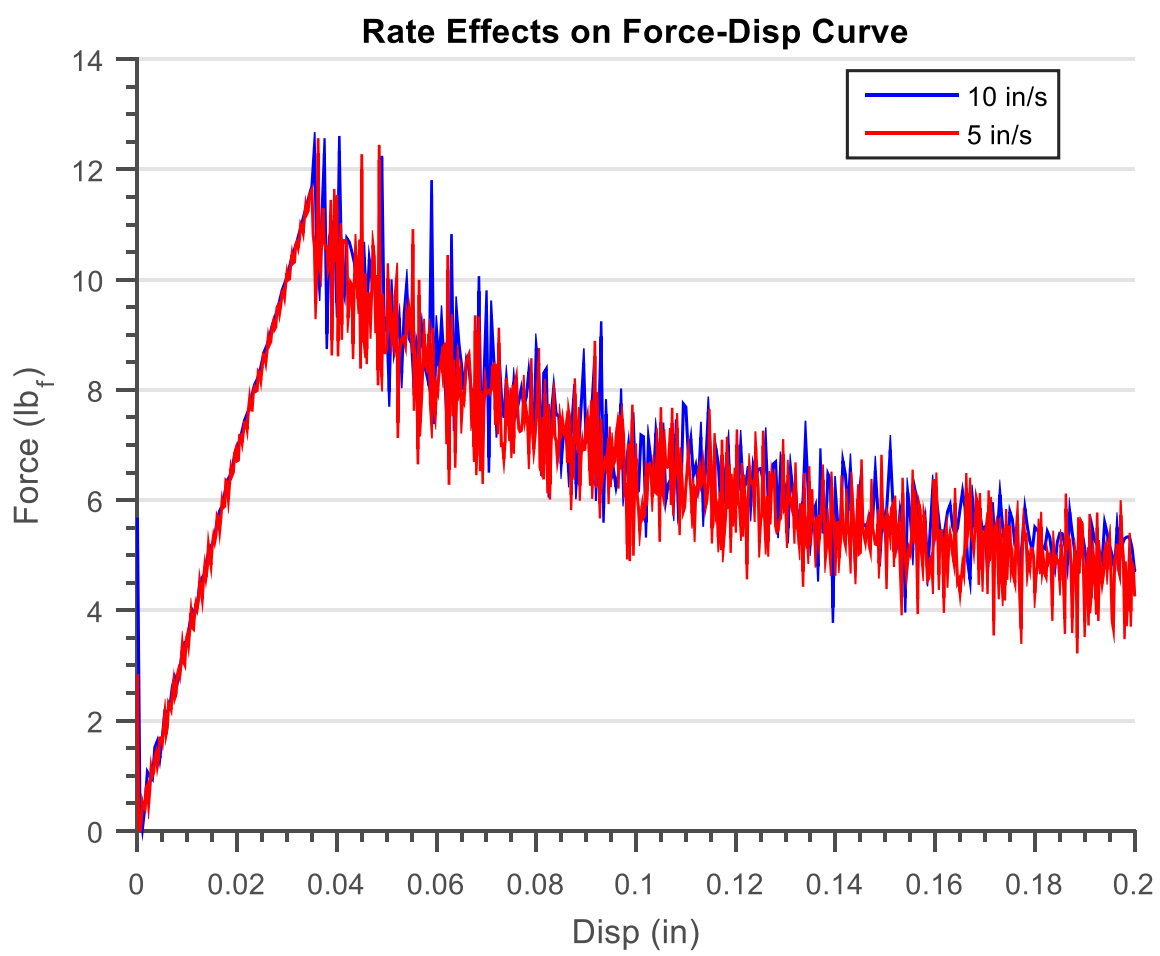

Figure 15 Rate dependency of DCB response prior to and after crack propagation

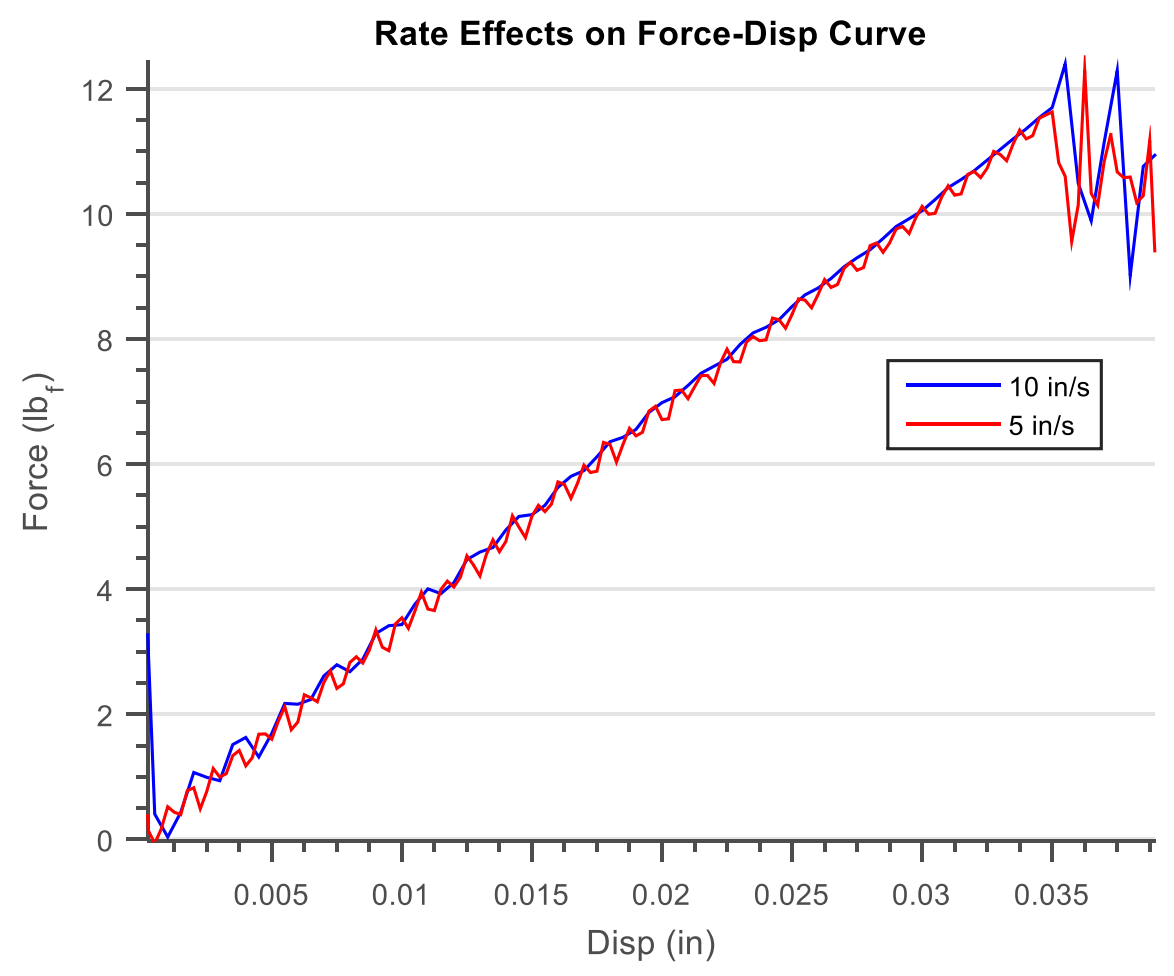

Figure 16 Rate dependency of DCB response up to crack propagation 
In order to achieve stable fracture as the crack began to propagate, the peak traction force was lowered until a steady crack growth response was achieved. Through many trials, it was found that a peak traction of 4000 psi gave reasonably steady fracture. This is shown in Figure 17. Larger peak traction stresses resulted in higher loads in the force-displacement curve but also gave unsteady crack growth. The blue and black curves in Figure 17 were characterized by crack growth followed by bending without crack growth, and then additional propagation of the crack. Steady crack growth is depicted with the green curve, with the load slowly falling off as the crack length increases. This finding was a major success in this research and took a large number of iterations.

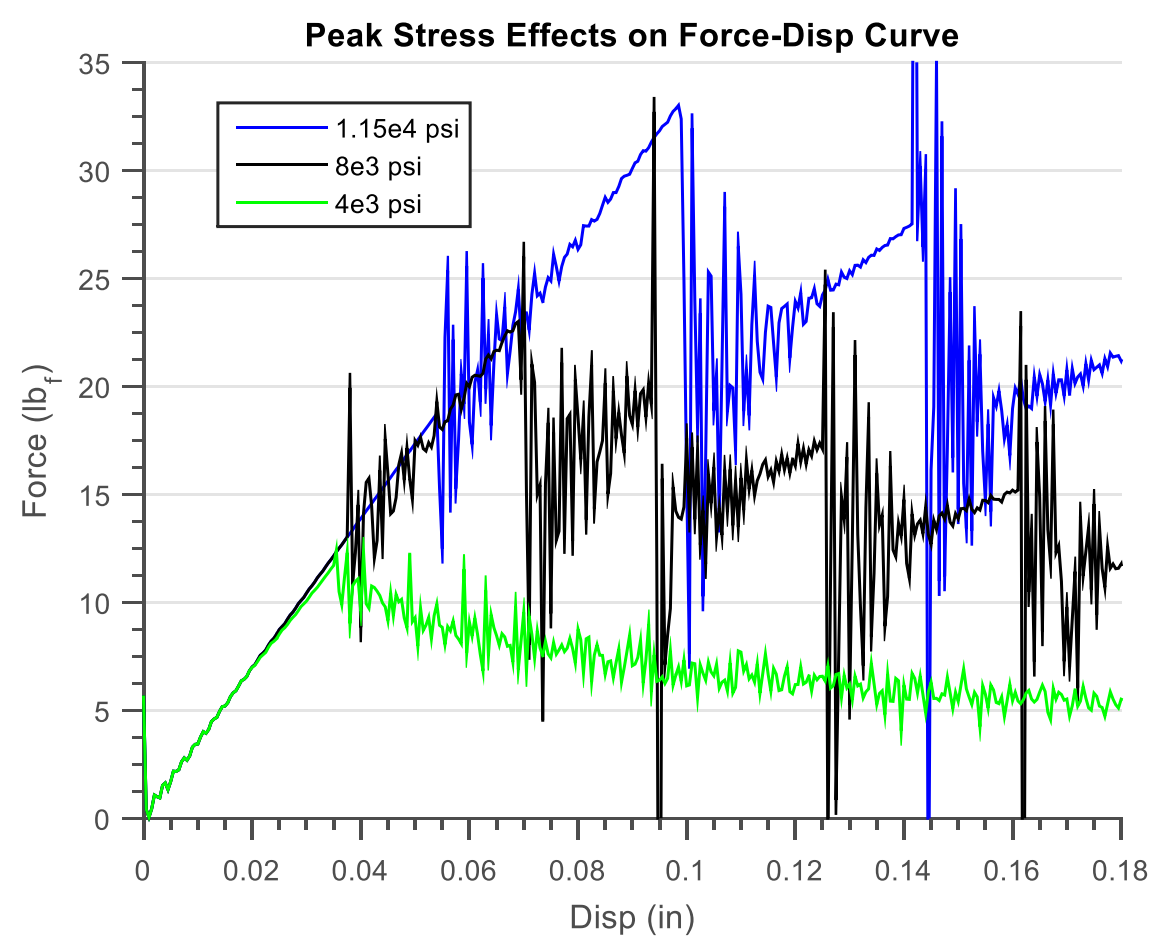

Figure 17 Peak traction stress effects on Mode I DCB force-displacement response

Mass damping effects, as mentioned in Section 2.2.3.5, are shown in Figure 18 and Figure 19 below. 


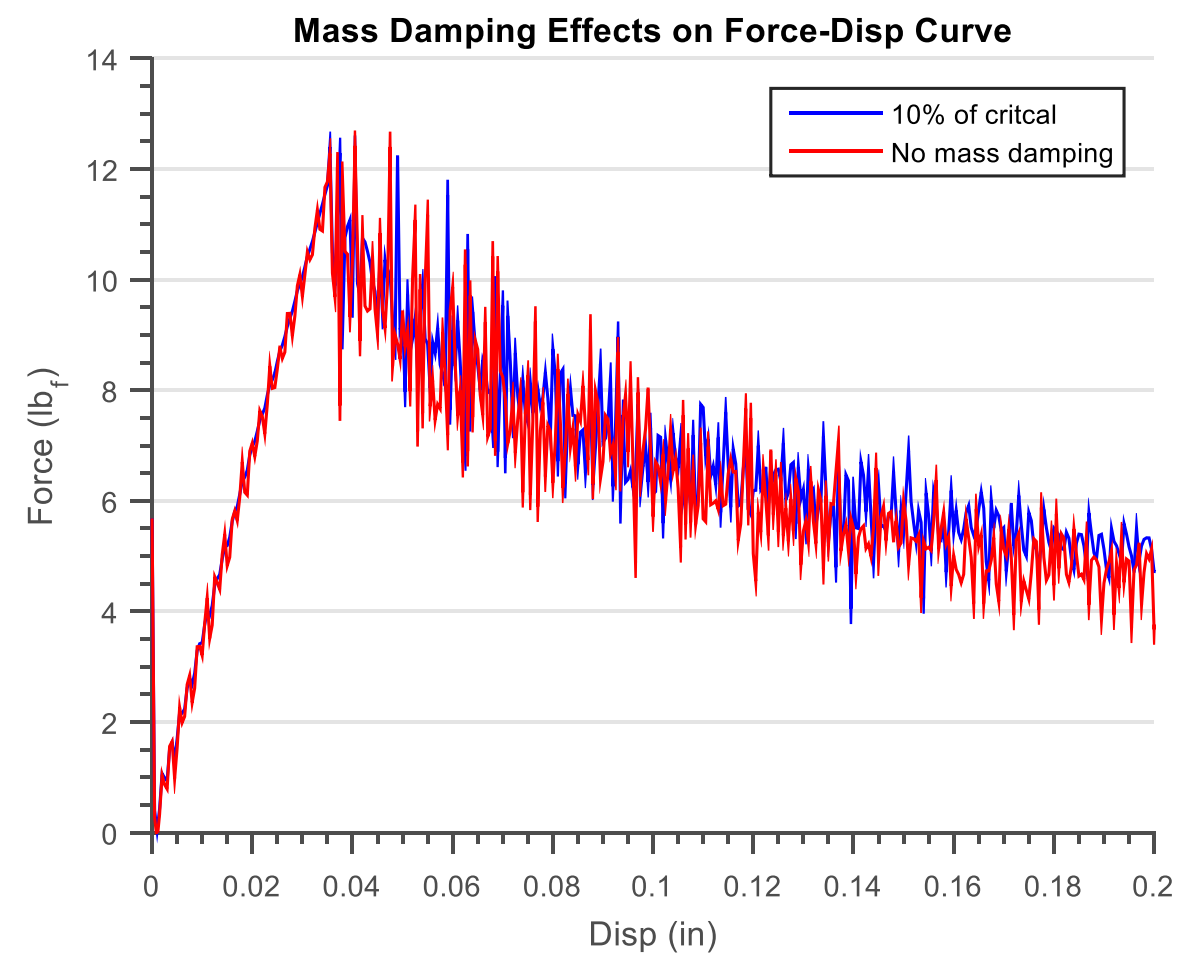

Figure 18 Mass damping effect on DCB response prior to and after crack propagation

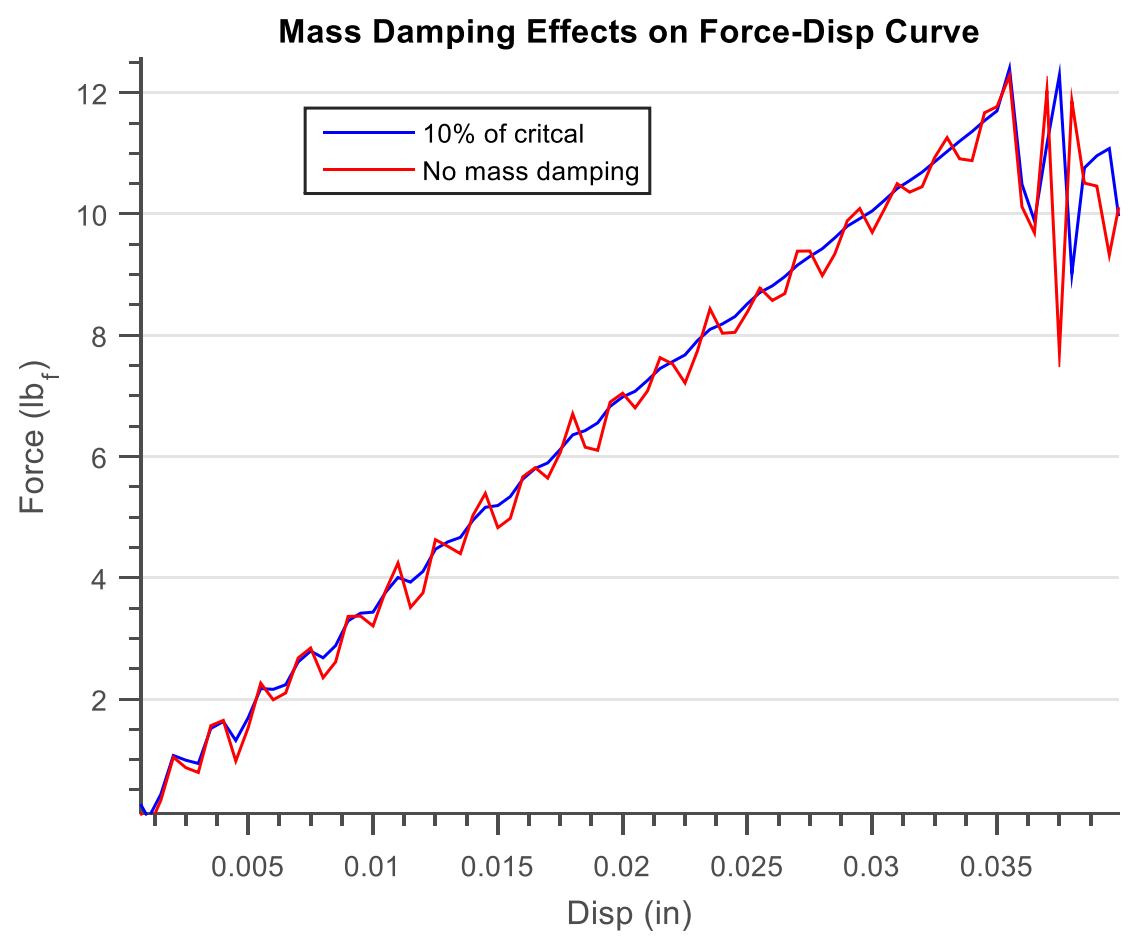

Figure 19 Mass damping effect on DCB response up to crack propagation 


\subsection{Laminated Carbon Fiber Plate Impact}

Using the lessons learned developing the Mode I fracture simulation, a model of a full carbon fiber laminated plate subjected to low velocity impact was created. The goal of the model was to capture the delamination initiation and propagation from the impact event. The interaction and initiation of other composite damage modes, such as fiber breakage or buckling, matrix cracking, or fiber pullout were not considered in this model. The focus was to determine the validity of using the contact tiebreak formulation to capture delamination propagation.

\subsubsection{Impact Model Geometry}

While there are many ways to model composites in finite element simulations, the three-dimensional nature of low-velocity impact lead to the use a full three-dimensional model. Delamination inherently arises from a three-dimensional state of stress within the layers of a laminate itself. The lay-up schedule for the laminate is given as $\left[00_{4} / 90_{4}\right]_{\mathrm{s}}$. The zero layers and ninety layers are each represented as their own respective sublaminate in the model. Rather than modeling each ply with a layer of three-dimensional elements or shell surfaces, each sublaminate (either all zeros or all nineties) was modeled with threedimensional elements with homogenized orthotropic properties of an orthotropic laminate. This is shown in Figure 20 where the steel impactor is the part in yellow, the top $\left[0_{4}\right]$ sublaminate is represented as the part in green, the middle $\left[90_{8}\right]$ sublaminate in blue, and the bottom $\left[\mathrm{O}_{4}\right]$ sublaminate in red. 


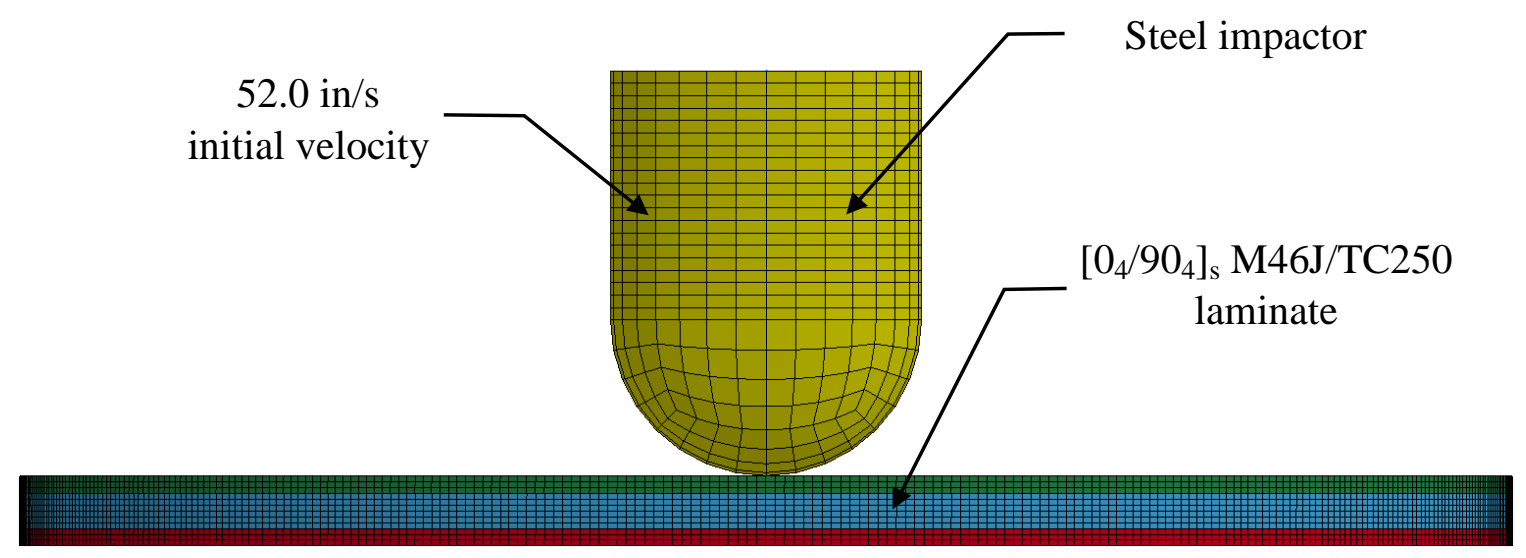

Figure 20 Laminated carbon fiber plate impact model: side view

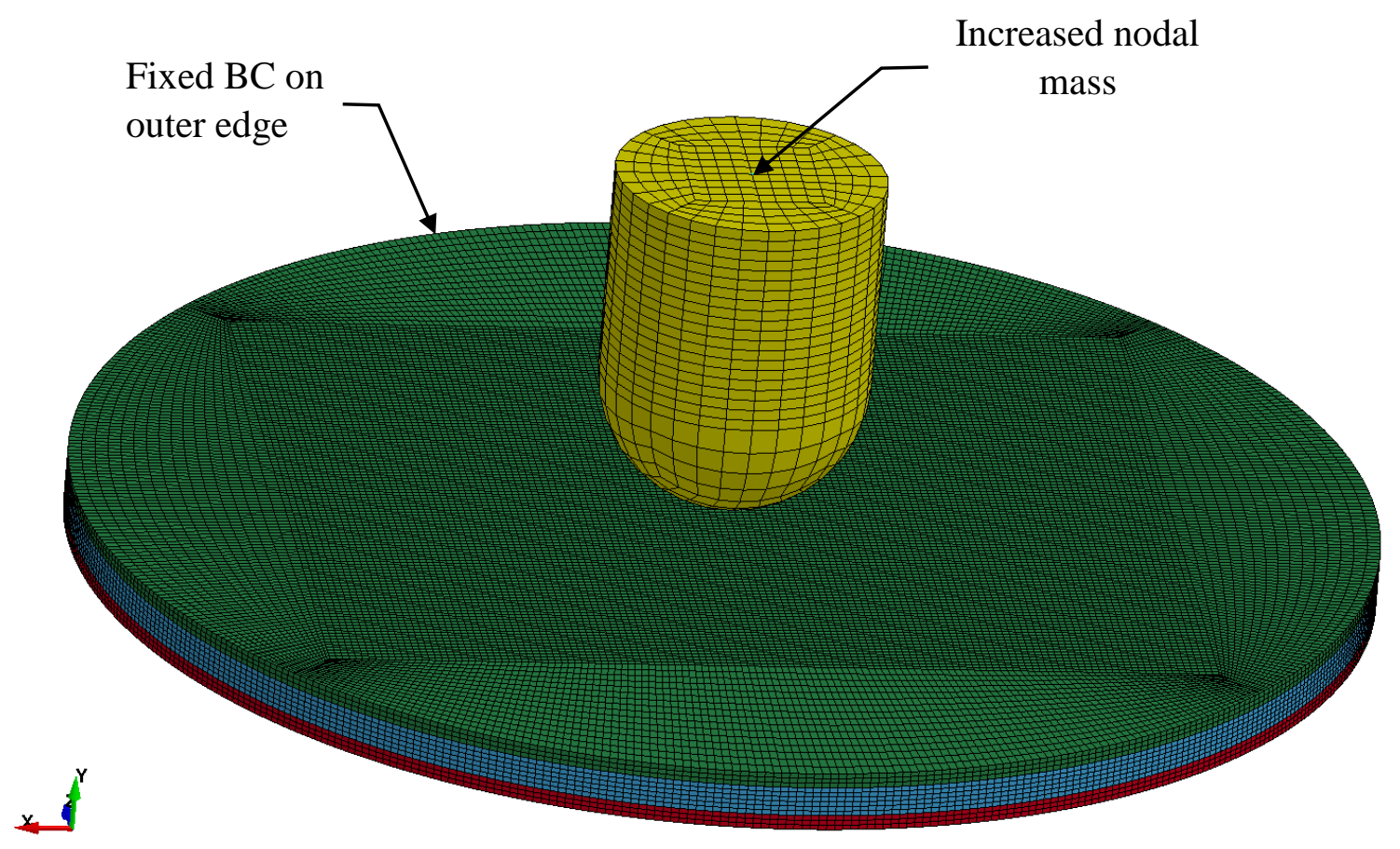

Figure 21 Laminated carbon fiber plate impact model: isometric view

The impactor has a total length of 0.8125 inches and the hemispherical impactor diameter is 0.625 inches. This was used to match the experimental impactor discussed in Chapter 3. The diameter of the laminated carbon fiber plate was modeled as three inches 
to mimic the clamping fixture geometry on the Dynatup. The mass of the impactor itself, along with its mounting nut and bolt, was measured to be $4.711 \times 10^{-4} \mathrm{lb}_{\mathrm{f}}-\mathrm{s}^{2} / \mathrm{in}$. However, this was not considering the mass of the carriage itself. The combined weight of the impactor, nut, bolt, and carriage with titanium bolts and aluminum weights was $9.021 \mathrm{x}$ $10^{-4} \mathrm{lb}_{\mathrm{f}}-\mathrm{s}^{2} / \mathrm{in}$. The additional mass was added via a nodal mass definition at the topmost central point on the impactor part, as shown in Figure 21. This gave the impactor the true inertia of the experimental testing in the drop weight impactor.

\subsubsection{Finite Element Parameters}

For specific model parameters, reduced LS-DYNA ${ }^{\circledR}$ keyword input files are included in Appendix C.

\subsubsection{Meshing \& Contact Tiebreak}

The mesh for the laminated carbon fiber plate and the steel impactor were created using TrueGrid ${ }^{\circledR}[28]$. A rectangular partition containing solid elements with an aspect ratio of one was created in the region where the contact tiebreak failure (delamination) develops. Figure 22 and Figure 23 show the model contoured by element aspect ratio and maximum distortion angle, respectively. Care was taken to ensure the model contained high quality elements, especially in the impact region. Just as in the Mode I simulation, a contact tiebreak definition was created between each sublaminate to allow for the delamination failure mode. Segments were assigned as explained in Section 2.2.3.1. 


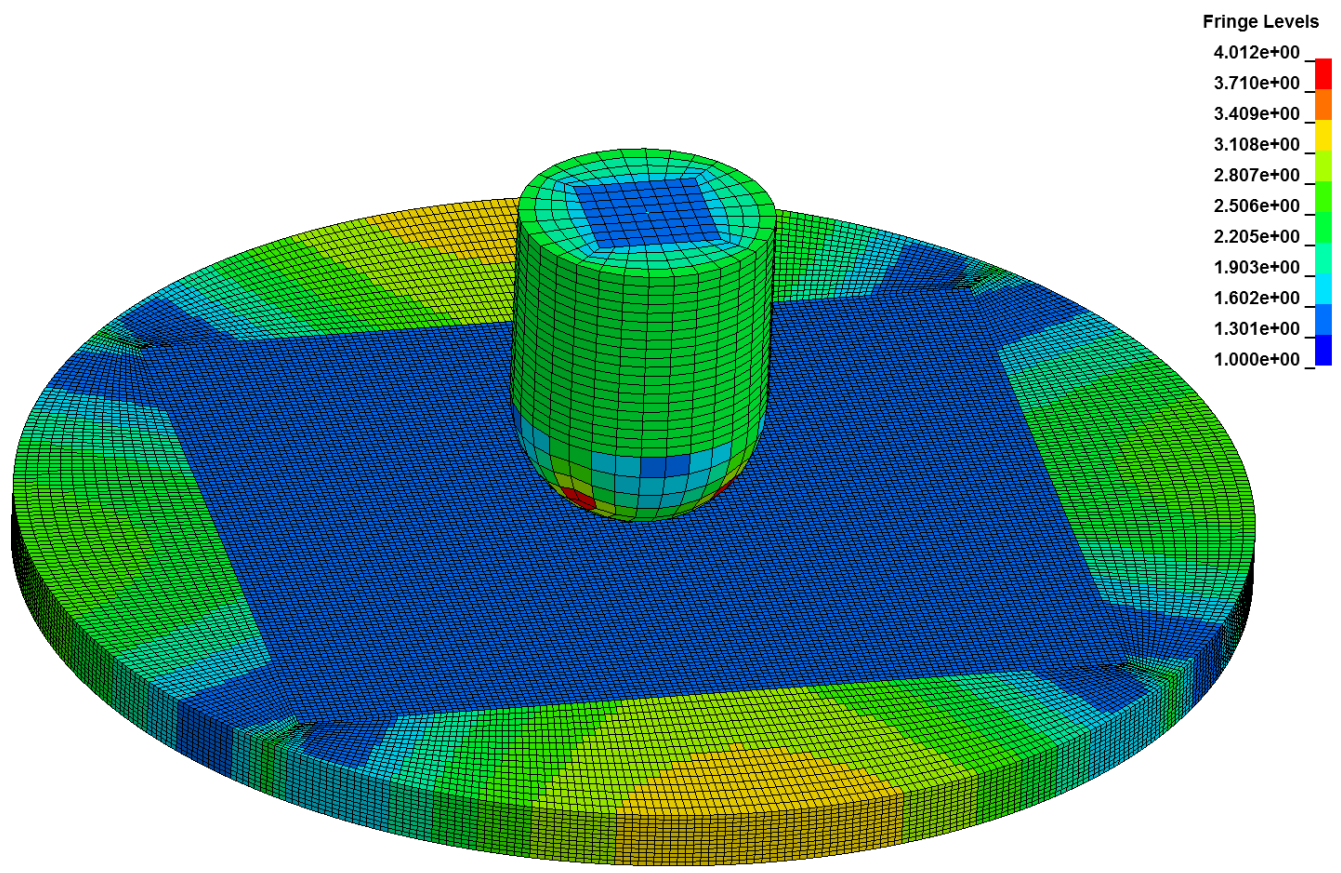

Figure 22 Aspect ratio of solid elements in impact model

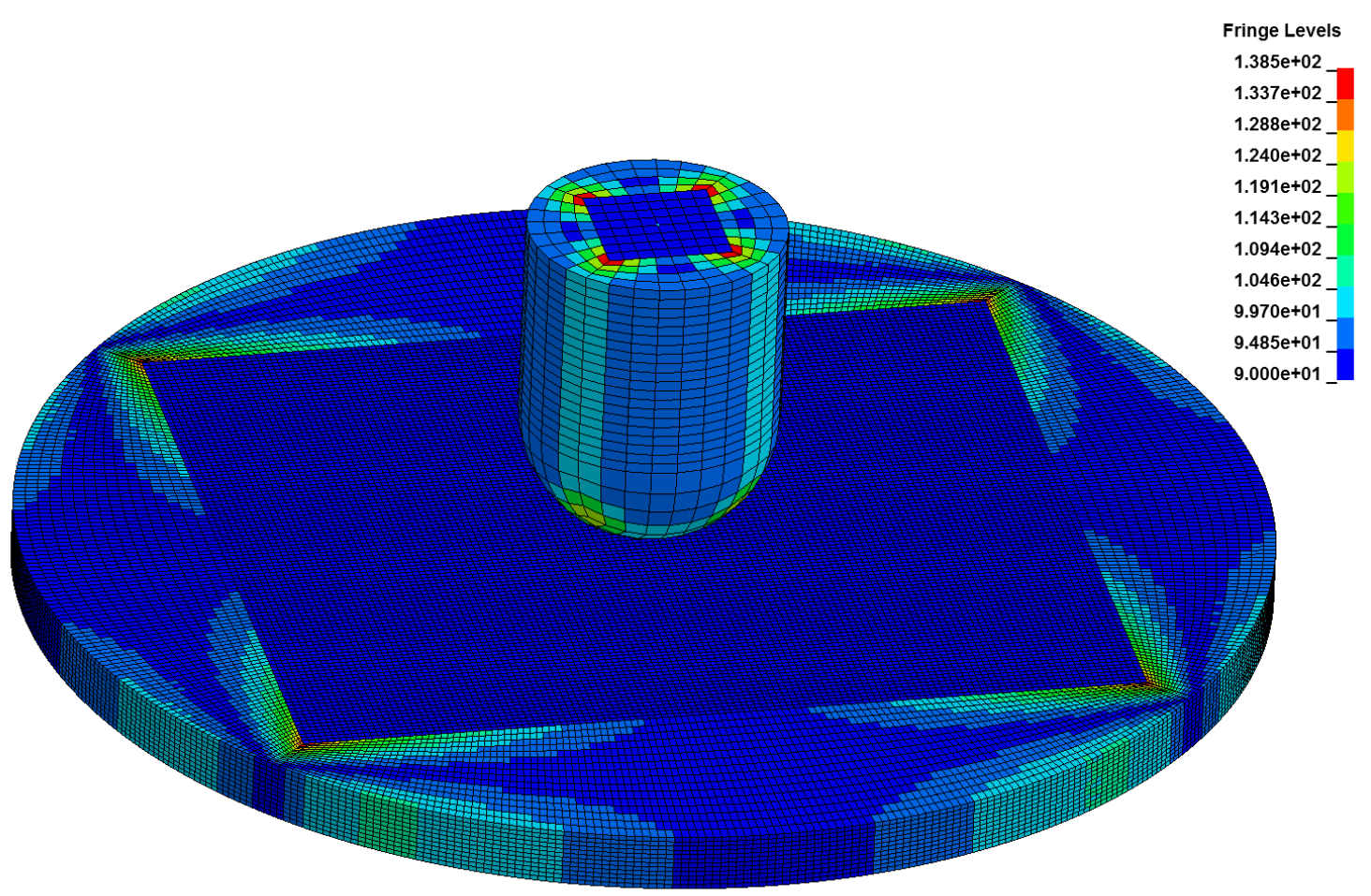

Figure 23 Maximum distortion angle of solid elements in impact model 
The contact tiebreak definition for the impact model utilized the almost all of the same properties as the Mode I simulation. Experimental testing for Mode I and Mode II fracture revealed that the M46J/TC250 likely had a fracture toughness that was much higher than expected [29]. Because of this, the energy release rate was increased from 0.7423 psi-in (as shown in Table 2) to 2.0 psi-in. While these properties are not the true normal and shearing fracture energy release rates $\left(\mathrm{G}_{\mathrm{I}}\right.$ and $\left.\mathrm{G}_{\mathrm{II}}\right)$, the resulting simulation gave reasonably sized delamination patterns. However, there were some unexpected location of delamination in the simulation and exact material and fracture properties are likely the major contributing factor in this discrepancy. Vibrothermography revealed the locations of internal delamination damage, as explained in Chapter 4.

\subsubsection{Element Type}

As explained in Section 2.2.3.2, solid element type 2 was selected which utilizes full integration and a selective reduced integration technique to improve element performance.

\subsubsection{Material Constitutive Response}

As in the Mode I model, the orthotropic-elastic Material Type 2 was utilized. Material properties from simple tensile specimen testing were utilized. These tests gave the fiber direction modulus $\left(\mathrm{E}_{1}\right)$, Poisson's ratio $\left(v_{12}\right)$, and shear modulus $\left(\mathrm{G}_{12}\right)$. In addition, material properties from the specification sheets for the M46J fibers and TC250 resin system were assumed [30, 31]. The laminate itself is made of M46J/TC250 unidirectional tape layers. Without complete knowledge of the material properties of the exact material system, assumptions for other properties were made using similar fiber/matrix combinations. The transverse modulus, $E_{2}$, was assumed from a TC250 
composite with different fibers with a similar volume fraction. This is a reasonable assumption since the transverse stiffness is dominated by matrix properties. Out-of-plane modulus, $E_{3}$, was assumed to be the same at $E_{2}$. The rest of the orthotropic elastic constants were determined based on the symmetry of transversely isotropic unidirectional laminates [2]. A composite material coordinate system is shown in Figure 24. Table 4 summarizes the composite material properties used for the impact simulation. The steel impactor was modeled using Elastic Material Type 1 with the properties given in Table 5.

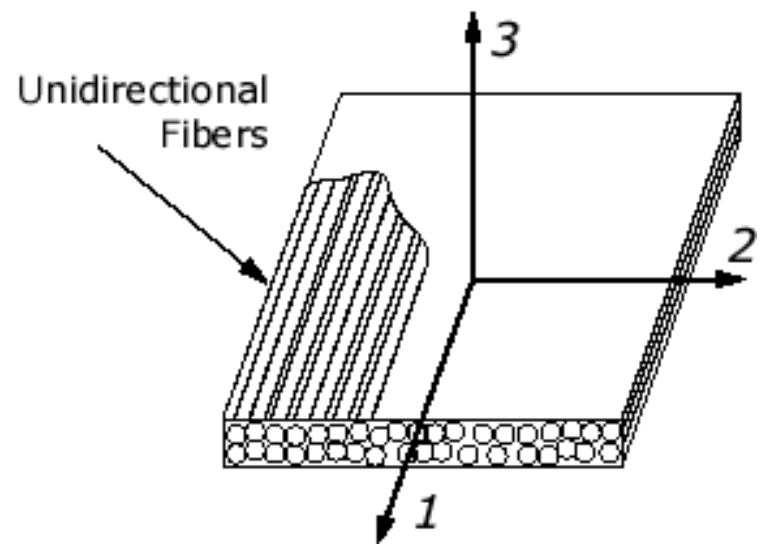

Figure 24 Composite material directions [32]

Table 4 M46J/TC250 orthotropic material properties in LS-DYNA ${ }^{\circledR}$

\begin{tabular}{|c|c|c|}
\hline Property & Value & Units \\
\hline $\mathrm{E}_{\mathrm{a}}$ & 29 & Msi \\
\hline $\mathrm{E}_{\mathrm{b}}$ & 1.42 & Msi \\
\hline $\mathrm{E}_{\mathrm{c}}$ & 1.42 & Msi \\
\hline$v_{\mathrm{ba}}$ & 0.011 & N/A \\
\hline$v_{\mathrm{ca}}$ & 0.011 & N/A \\
\hline$v_{\mathrm{cb}}$ & 0.3000 & N/A \\
\hline $\mathrm{G}_{\mathrm{ab}}$ & 0.4770 & Msi \\
\hline $\mathrm{G}_{\mathrm{bc}}$ & 0.4770 & Msi \\
\hline $\mathrm{G}_{\mathrm{ca}}$ & 0.4770 & Msi \\
\hline$\rho$ & $1.349 \mathrm{E}-04$ & $\mathrm{lbf}-\mathrm{s}^{2} / \mathrm{in}$ \\
\hline
\end{tabular}


Table 5 Steel impactor material properties in LS-DYNA ${ }^{\circledR}$

\begin{tabular}{|c|c|c|}
\hline Property & Value & Units \\
\hline E & 30 & Msi \\
\hline$v$ & 0.30 & N/A \\
\hline$\rho$ & $7.15 \mathrm{E}-04$ & $1 \mathrm{bf}-\mathrm{s}^{2} / \mathrm{in}$ \\
\hline
\end{tabular}

\subsubsection{Boundary Conditions}

The outer edge of the three inch diameter plate was modeled with a fixed boundary condition. This was used to simulate the clamped edge conditions of the experimentally impacted plate. While the experimental clamping fixture had an additional notch that was not modeled (shown in Figure 35), the necessary partitioning and large number of elements for a quality mesh with the notch included was prohibitively large.

\subsubsection{Damping}

Since the impact model did not contain a built in delamination as in the Mode I model, damping was determined to be unnecessary. A small amount of part stiffness damping equal to $1 \%$ of critical damping was added.to this model.

\subsubsection{Results}

In order to compare the finite element simulation to the experimental impact testing discussed in Chapter 3, the acceleration of a point on the impactor was tracked over each time step in the simulation. Since the mass of the carriage was lumped at the topmost central point on the impactor, the acceleration of this nodal point was plotted for each time step. Figure 25 overlays the unfiltered experimental force vs. time curve and the force vs. time computed in the simulation for a $12.2 \mathrm{lb}_{\mathrm{f}}$-in impact energy event. The contact time for the simulated impact is slightly shorter than the experimental contact 
time. This means that the laminate is likely less compliant than the real laminate. Since the composite material model was linear elastic without failure, the only failure mode in the laminate is delamination. Damage from physical impact resulted in some matrix cracking as well as indentation and local cracking in the impact location. Each of these damage modes increase the compliance of the laminate and increase the amount of time that the impactor is contacting the laminate.

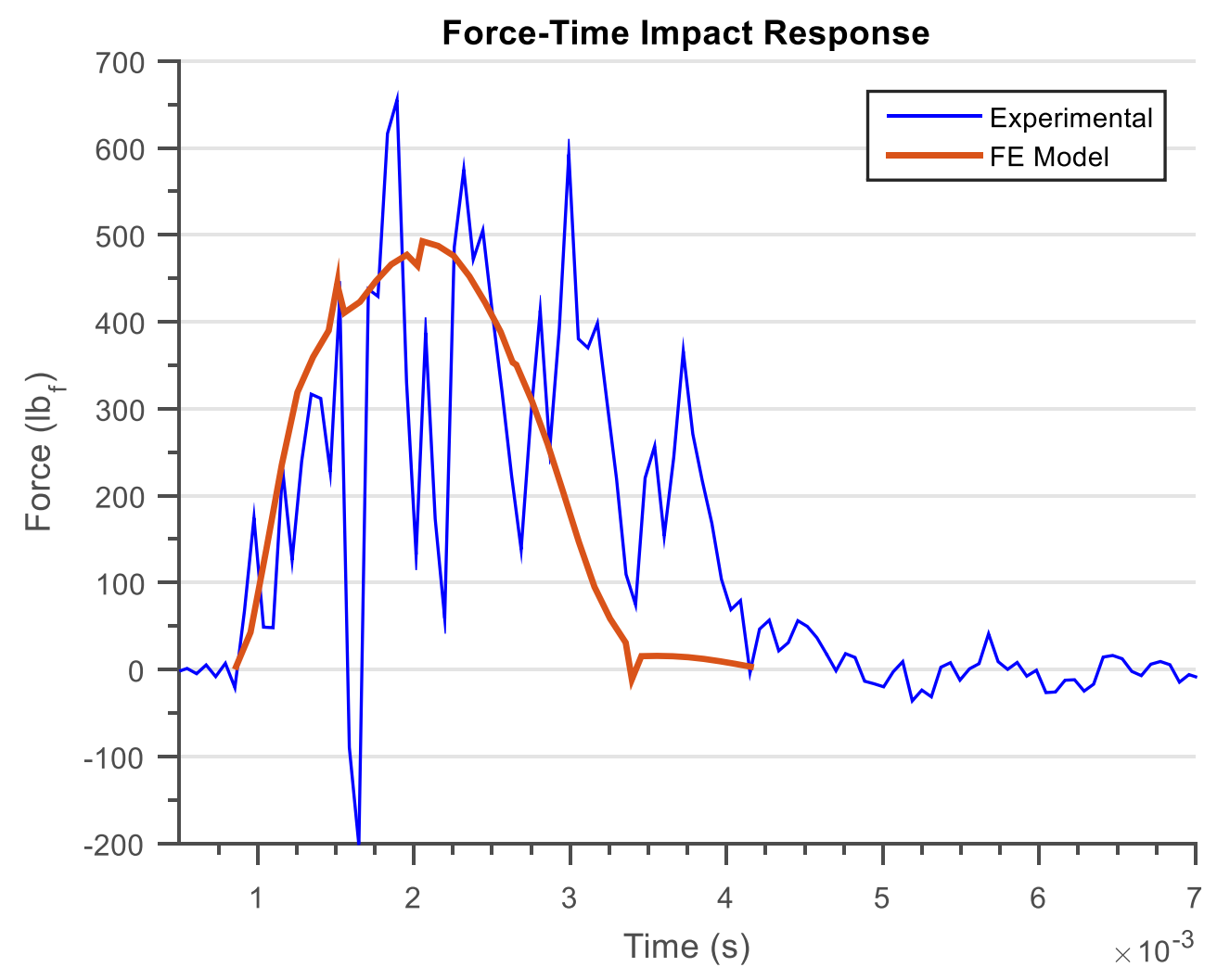

Figure 25 Force-time response of

Some of the delamination damage developed during the impact event was captured in the simulation and is shown in Figure 26 and Figure 27. These figures are section planed views of the laminate showing internal delamination damage between sublaminates. The left side of Figure 26 shows shearing failure in the xy-plane between 
the top (fibers in $\mathrm{x}$-direction) and middle (fibers in $\mathrm{z}$-direction) sublaminates. The sublaminates slide past each other when the peak shear traction force is reached in the contact tiebreak formulation and exhibit normal separation when the peak normal traction is reached.

Figure 44 shows the theoretically predicted damage shapes between the top and middle sublaminates and the middle and bottom sublaminates, respectively. The expected orientation of the delaminations at the upper and lower sublaminate interfaces was confirmed using vibrothermography, as shown in Figure 45. However, finite element modeling of delamination damage in this laminate only partially agreed with experimental testing. In order to determine the damaged regions where delamination had occurred, a script was written to post-process the finite element simulation results. While LS-DYNA ${ }^{\circledR}$ has a the built in capability to write contact tiebreak damage output files, this capability was not available with the version of the software utilized for this research. In lieu of the built in damage post-processing features, the Matlab ${ }^{\circledR}$ script analyzed the separation (both tangential and normal) between the interfaces of sublaminates in the laminate. The final distance between each node that was originally tied together was computed in the normal and tangential directions. This was used as a suitable indicator for the relative amount of normal and shearing delamination that occurred during the simulation, as well as the location of that damage. Contour plots of the post-processed damage are shown in Figure 28 through Figure 31. 


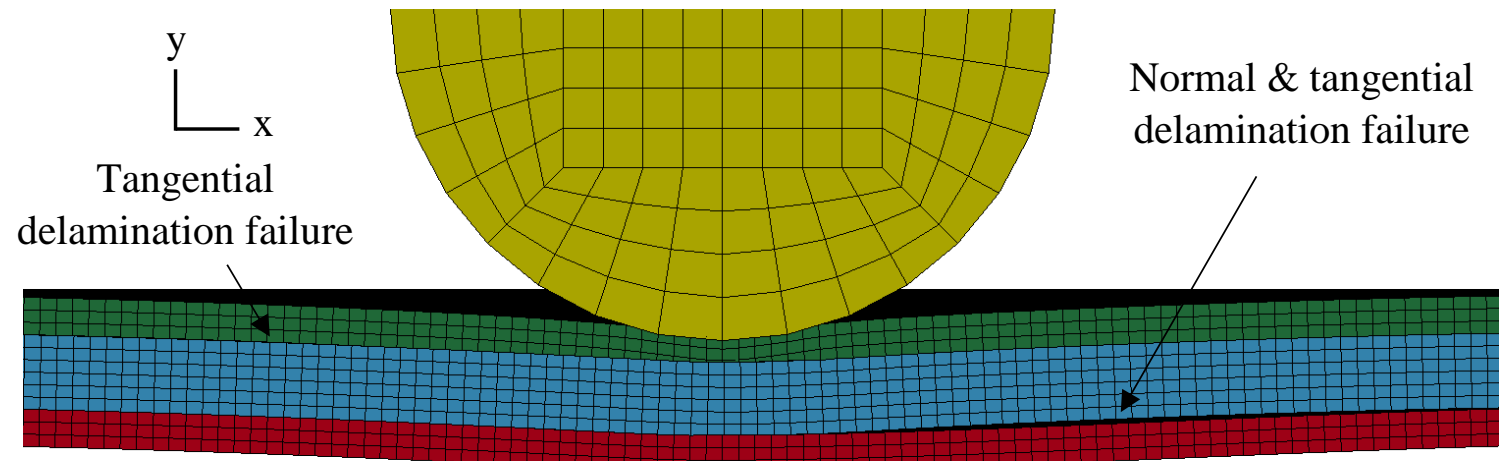

Figure 26 Delamination failure between sublaminates in $x-y$ plane

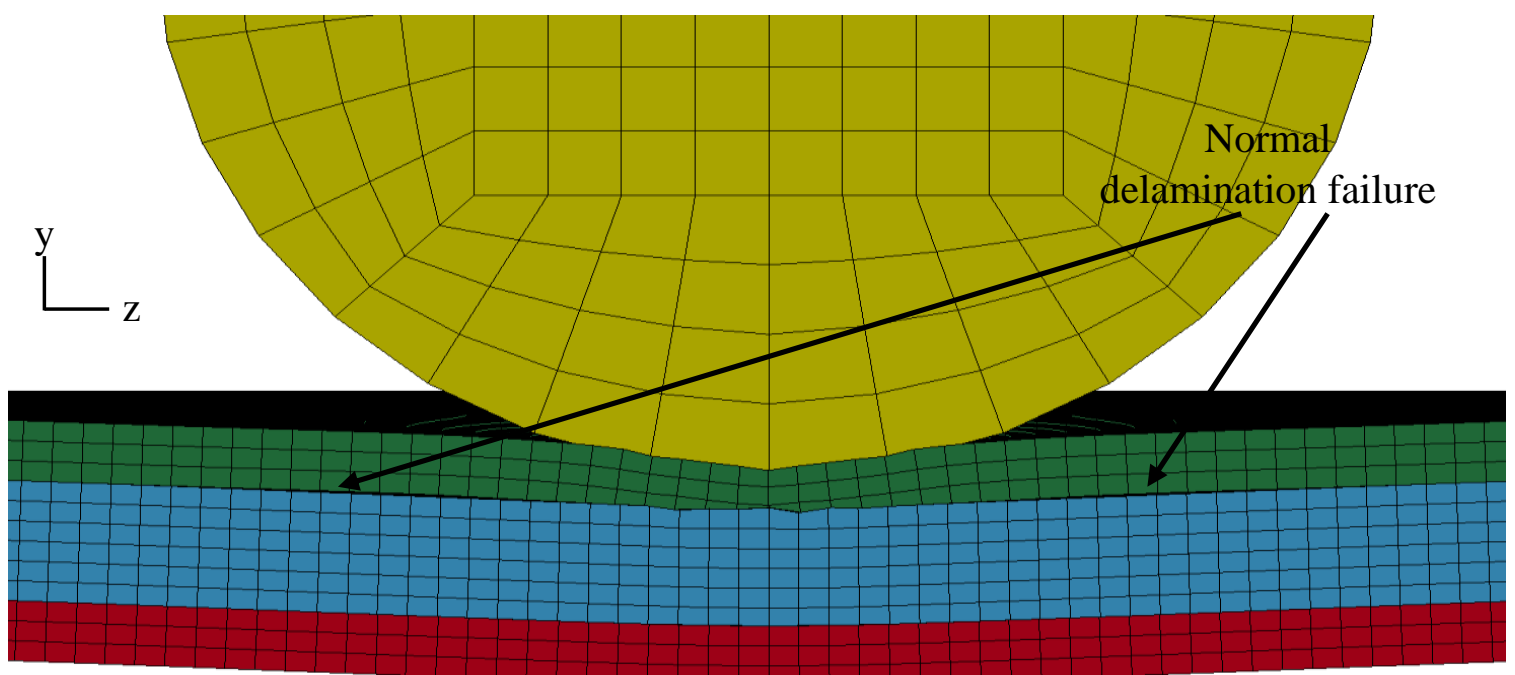

Figure 27 Delamination failure between sublaminates in y-z plane

The normal delamination plot for the uppermost sublaminate interface, given in

Figure 28, matches very well with the relative predicted size and shape of the delamination at this interface. In addition, the vibrothermography experimentation revealed a similar delamination pattern shown on the right of Figure 45. The shearing damage at this interface of Figure 29, however, does not agree with experimental or predicted delamination. Many experiments, including those in this research, have shown 
that the major axis of the peanut-shaped delamination between plies with different orientations aligns with the sublaminate fiber orientation that is farther from the impact surface. In the case of the $\left[00_{4} / 90_{8} / 0_{4}\right]$ laminate, the first $[0 / 90]$ ply interface should contain a delamination that is oriented in the direction parallel to the $90^{\circ}$ fibers. The additional lobe shaped shear failure on the left side of Figure 29 was not seen in the vibrothermography imaging experiments. Interestingly, if this shear delamination were present in the lower sublaminate interface shown in Figure 31, this would match almost exactly with the predicted delamination pattern seen by Finn and Springer for the same laminate, which is shown in Figure 32 [5]. The asymmetry of the two horizontal lobes seen in the experimental vibrothermography (Figure 49), literature (Figure 32), and finite element simulation (Figure 30 and Figure 31) do agree, which shows the development of the correct delamination trend in the simulation.

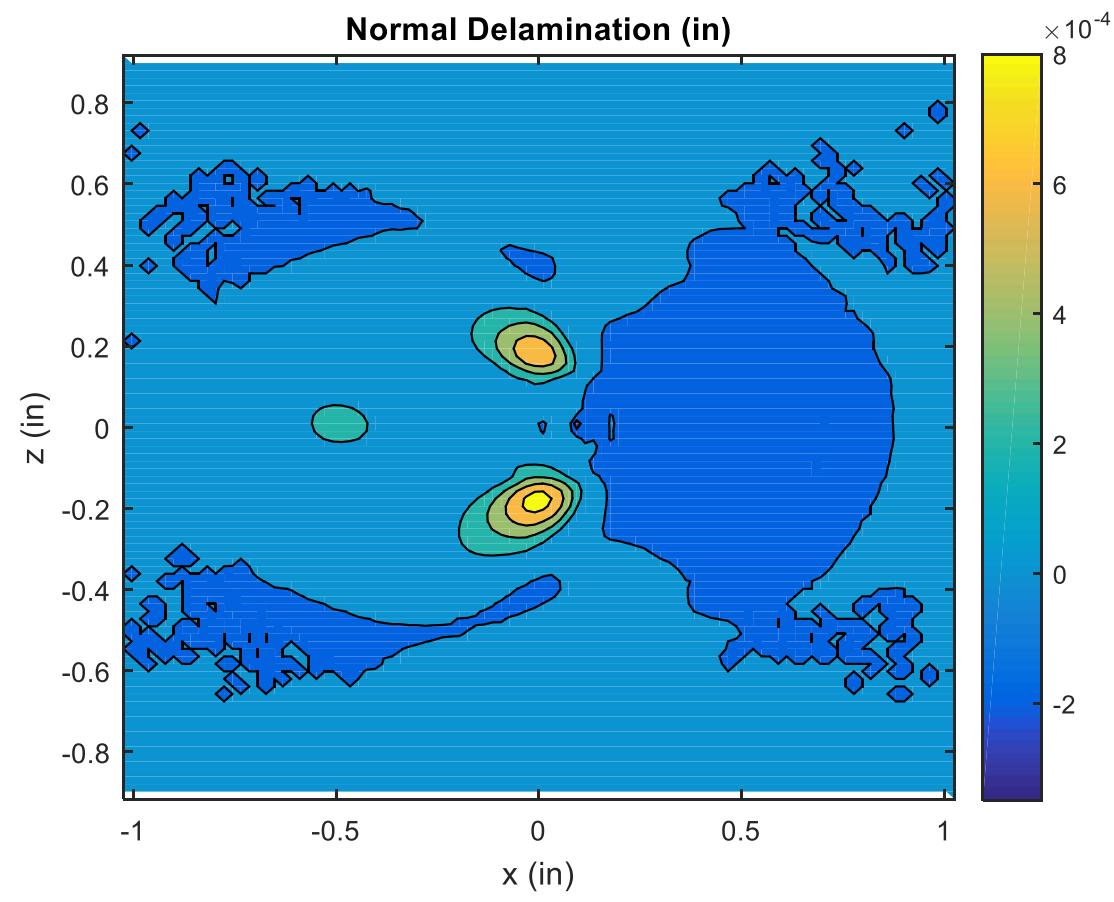

Figure 28 Contour plot of separation damage between top and mid sublaminates 


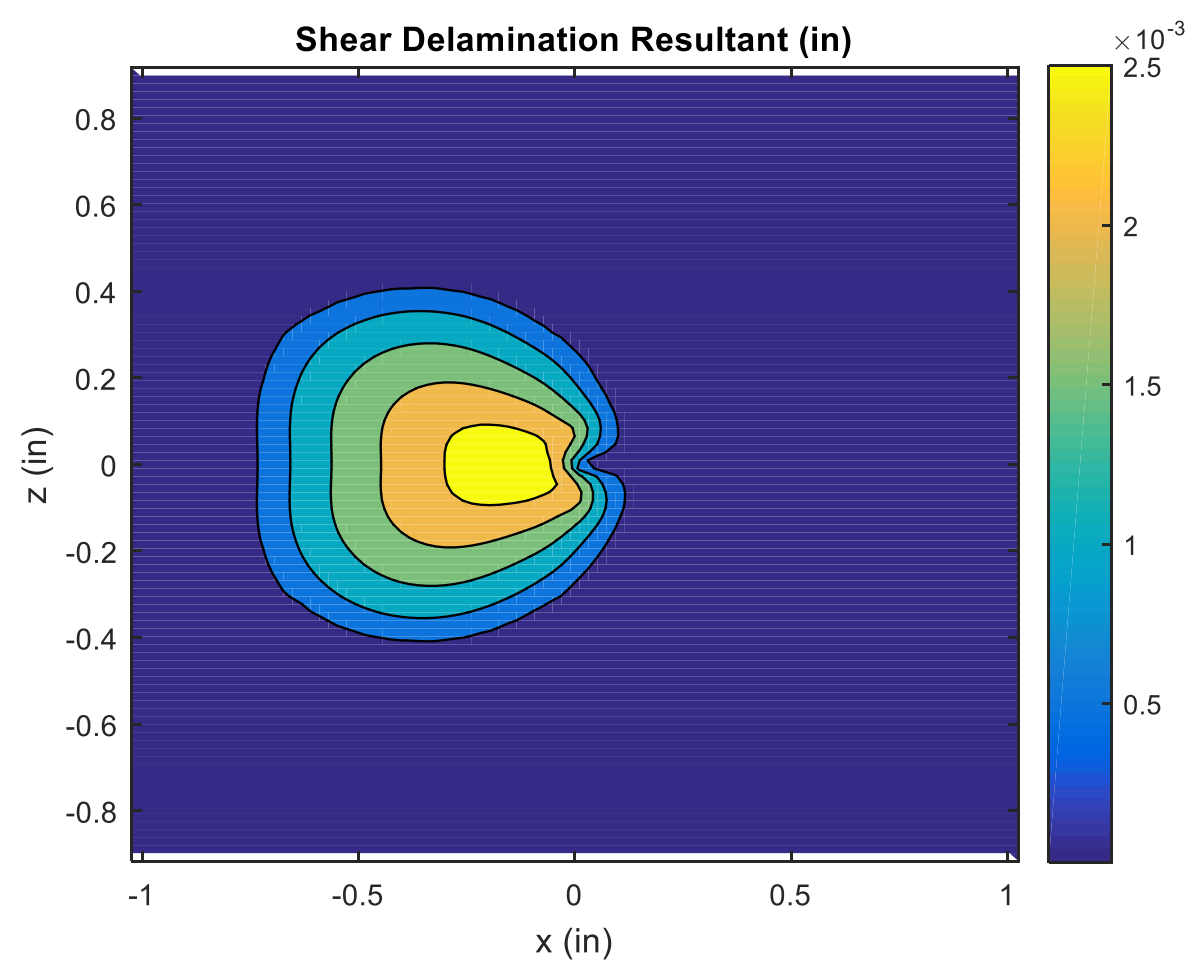

Figure 29 Contour plot of shear separation damage between top \& mid sublaminates

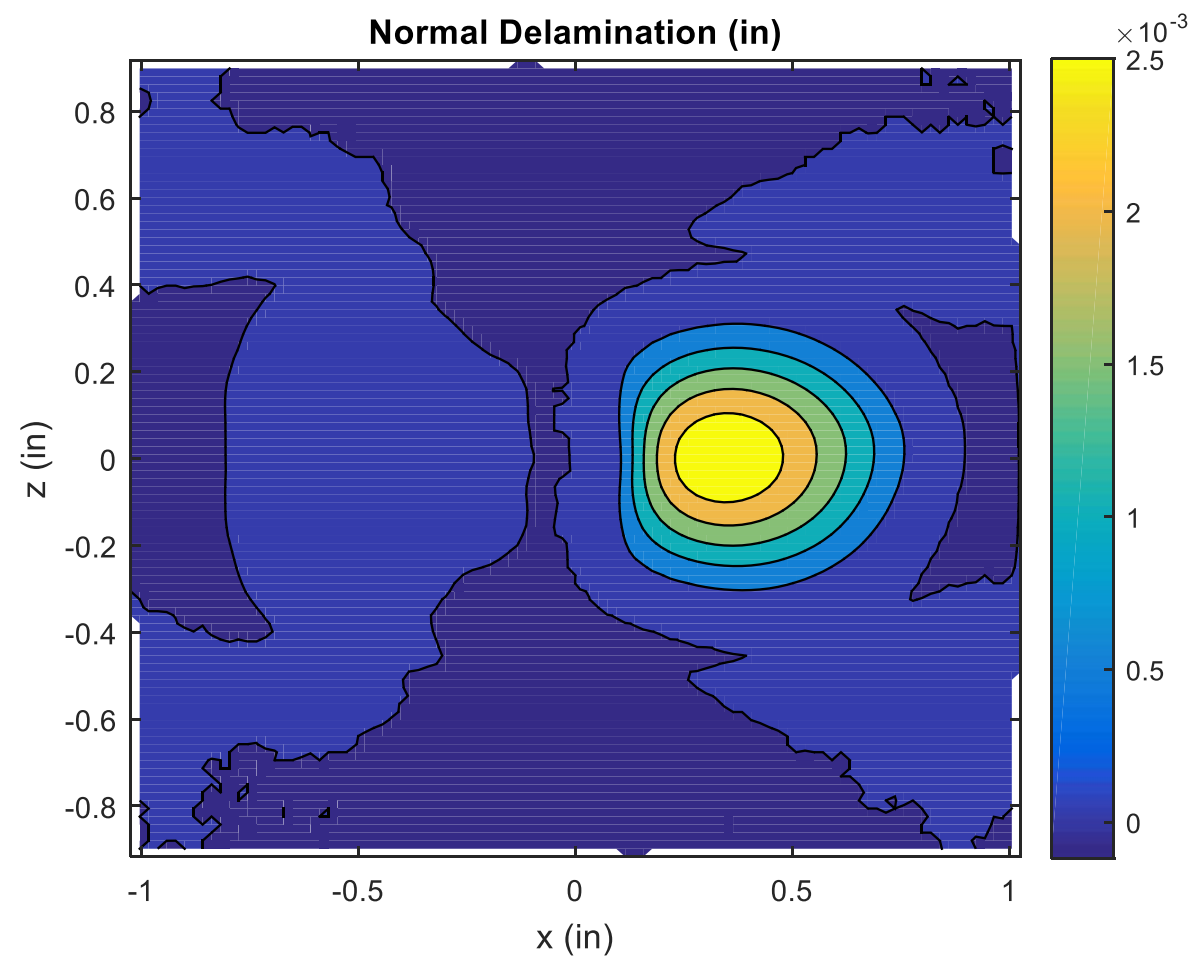

Figure 30 Contour plot of normal separation damage between mid \& bot sublaminates 


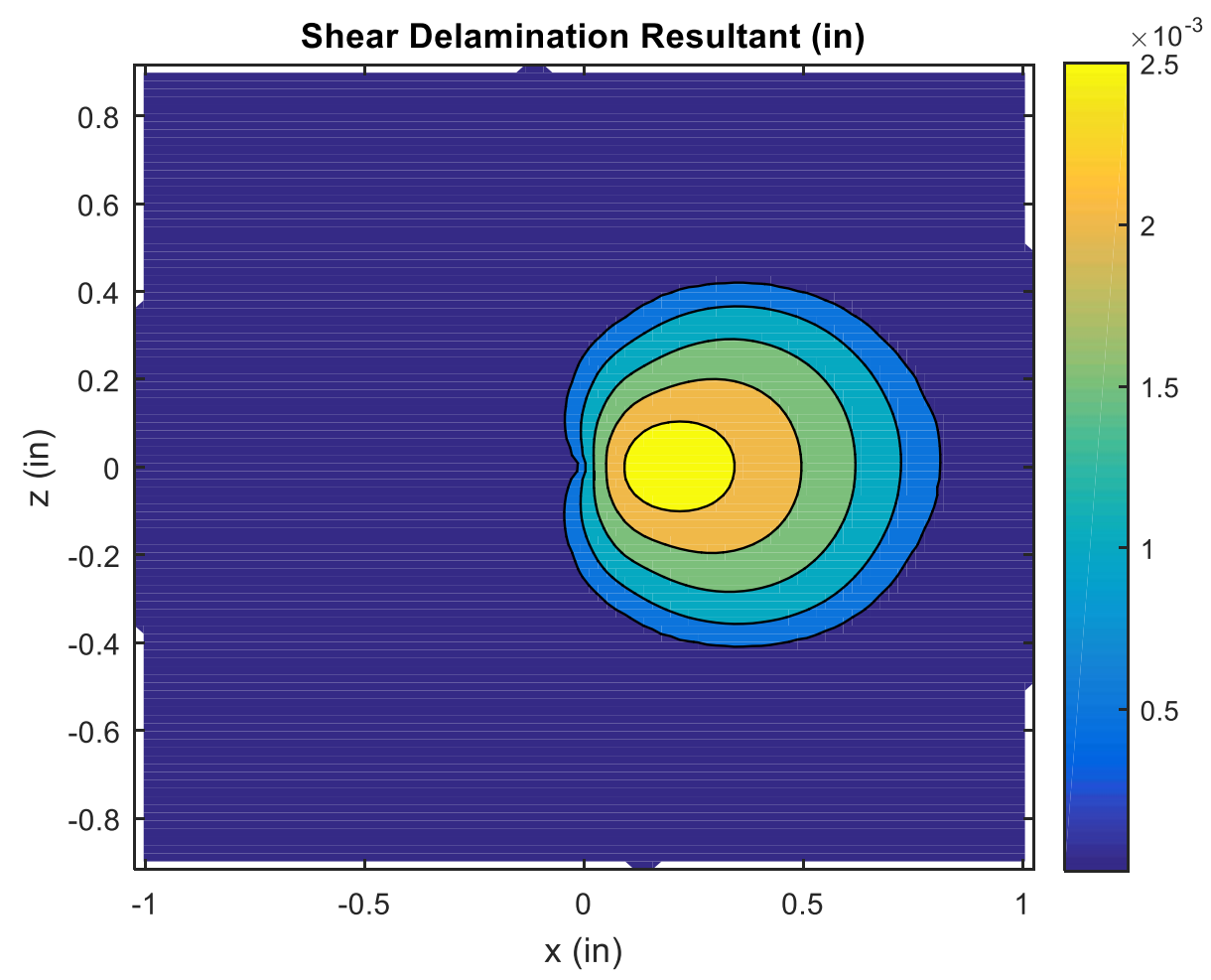

Figure 31 Contour plot of shear separation damage between mid \& bot sublaminates

However, a discrepancy between physical testing and the simulation is present. This is likely due to a combination of assumed fracture energy properties and the lack of other failure modes in this simulation. The interaction between failure modes such as fiber failure, matrix cracking, and delamination is very complex and hard to predict. The consequence of using an elastic orthotropic composite material model without failure is that these interactions were not captured. Additionally, the assumed fracture properties for the contact tiebreak definition were based on very limited experimental testing. It is likely that the mixed-mode nature of impact delamination requires different fracture energies and peak traction stresses for Mode I and Mode II failure. 


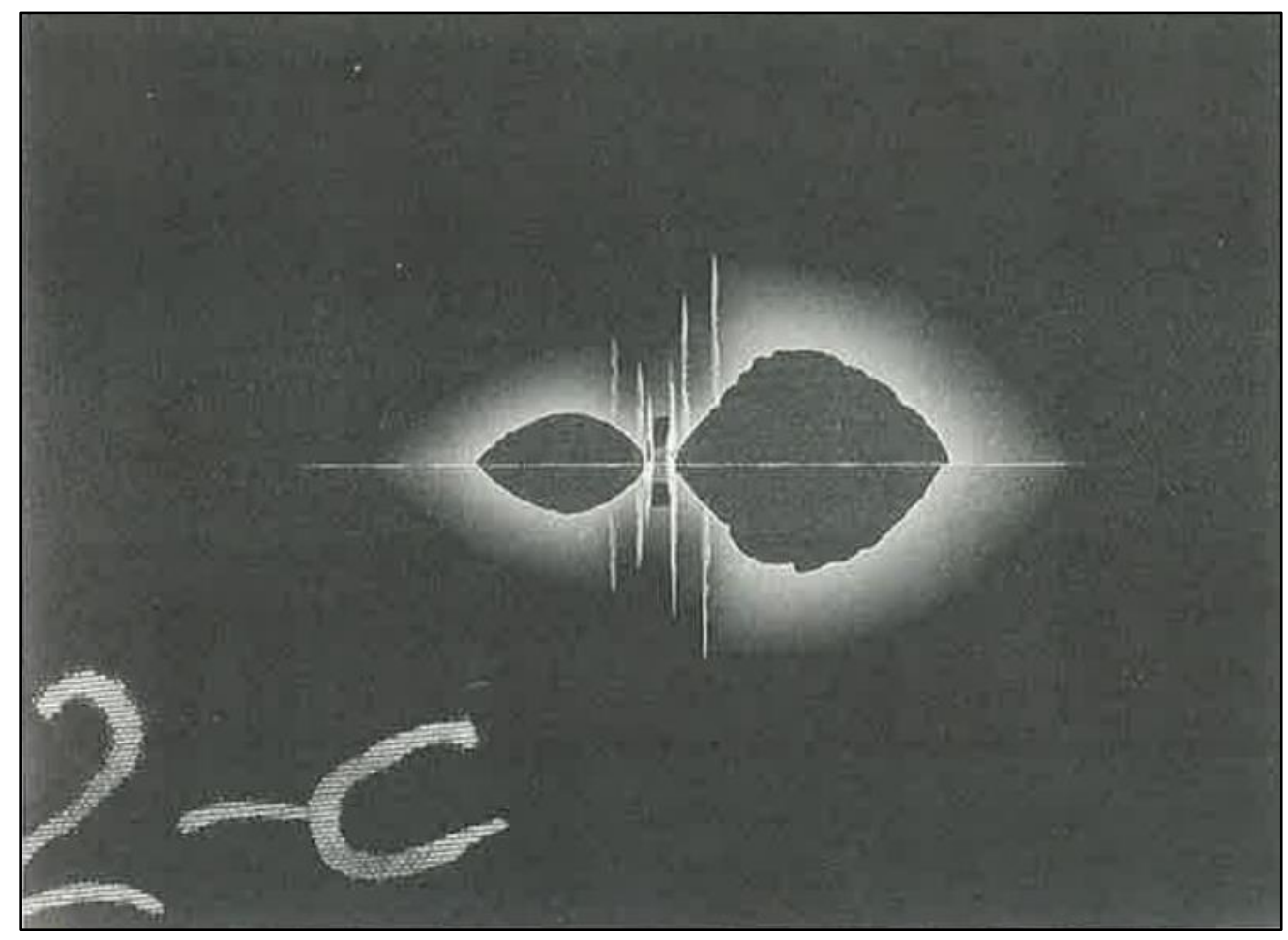

Figure $32 \mathrm{X}$-ray of T300/976 [04 $\left./ 90_{4}\right]_{\mathrm{s}}$ plate quasi-impact loaded with $13.4 \mathrm{lb}_{\mathrm{f}}$-in [5] 


\section{CHAPTER 3: EXPERIMENTAL IMPACT TESTING}

\subsection{Specimen Manufacturing}

The carbon fiber reinforced epoxy laminates utilized unidirectional prepreg with M46J high modulus fibers and a TC250 resin system. While this material was past its rated shelf life in the freezer, it was chosen due to its large supply and was assumed to have little degradation in its mechanical properties. Mechanical testing for $\mathrm{E}_{1}, \mathrm{G}_{12}$, and $v_{12}$ properties were determined using simple tensile test specimens. Each laminate was cured at $250^{\circ} \mathrm{F}$ under a $25-30 \mathrm{~mm}-\mathrm{Hg}$ vacuum and then cut to size using a tile saw. An approximate cure cycle for this material system is shown in Figure 33.

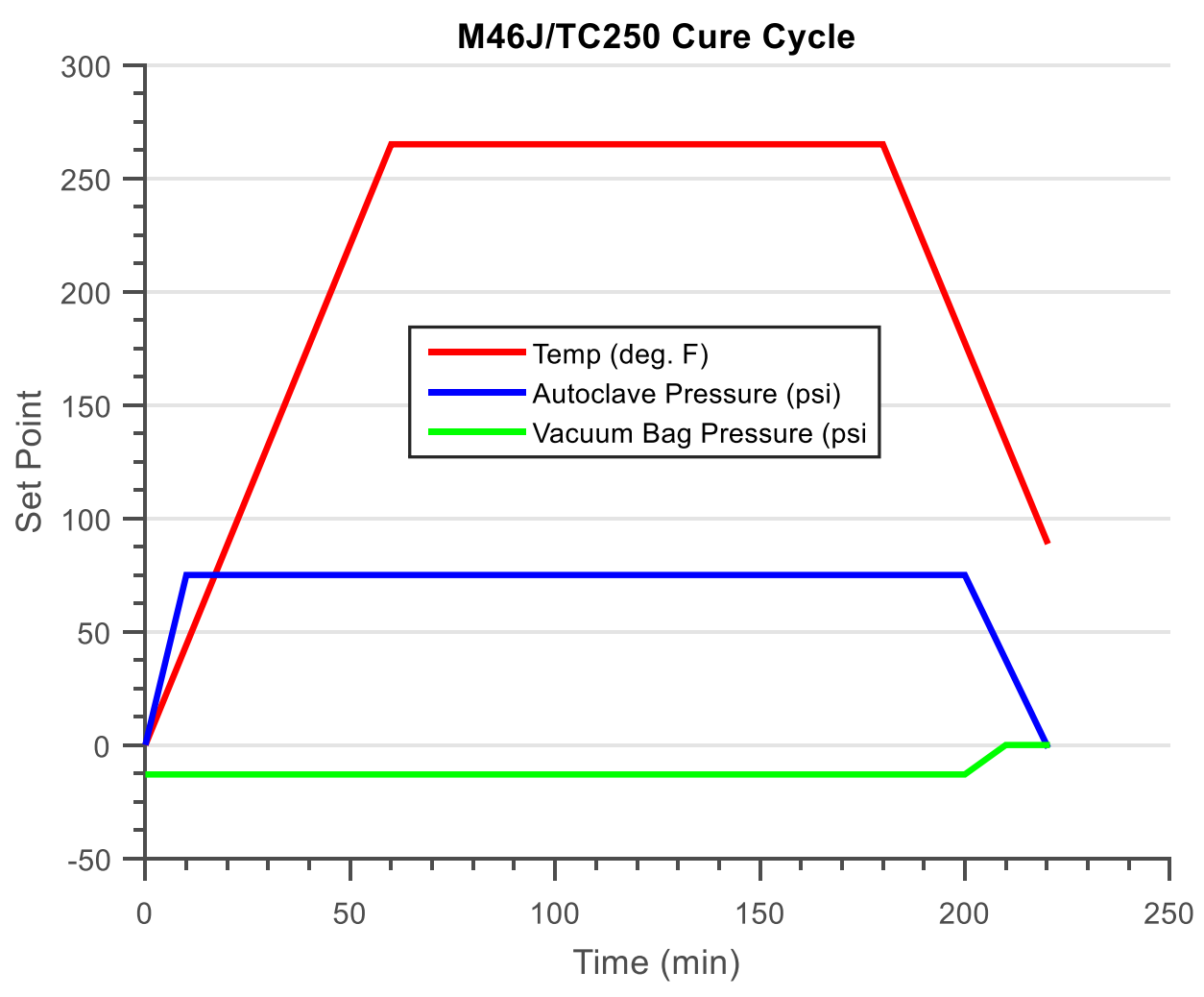

Figure $33 \mathrm{M} 46 \mathrm{~J} / \mathrm{TC} 250$ cure cycle 


\subsection{Impact Test Set Up}

Impact testing was performed using an Instron Dynatup 8250 drop weight impact test machine (Figure 34). The impact test apparatus utilizes a steel hemispherical impactor attached to a rail-guided carriage. The carriage consists of two crossheads that can be attached with plates of varying weight. This allows different impactor energies to be used based on which plate is chosen. This can also be seen in Figure 34. Lightweight titanium plates with aluminum screws were used to attach the two crossheads to achieve a low impact energy and control the size of the delaminations created during the impact event. Table 6 outlines the key parameters of the impact experiments. Impact energy and initial velocity prior to impact were calculated theoretically based on the height the impactor drop height of each test.

Table 6 Summary of impact testing parameters

\begin{tabular}{|c|c|c|c|c|c|c|c|}
\hline Test & Material & Laminate & $\begin{array}{l}\text { Thickness } \\
\text { (in) }\end{array}$ & $\begin{array}{l}\text { Drop } \\
\text { Height } \\
\text { (in) }\end{array}$ & $\begin{array}{c}\text { Mass } \\
\left(\mathbf{l b}_{\mathrm{f}}-\mathrm{S}^{2} / \mathrm{in}\right)\end{array}$ & $\begin{array}{l}\text { Energy } \\
\left.\text { (lb } b_{f}-i n\right)\end{array}$ & $\begin{array}{c}\text { Velocity } \\
\text { (in/s) }\end{array}$ \\
\hline 1 & M46J/TC250 & {$\left[0_{7} / 90_{7}\right]$} & 0.12 & 2.7 & $9.02 \mathrm{E}-03$ & 9.4 & 45.7 \\
\hline 2 & $\mathrm{M} 46 \mathrm{~J} / \mathrm{TC} 250$ & {$\left[0_{4} / 90_{4}\right]_{\mathrm{s}}$} & 0.14 & 3.5 & $9.02 \mathrm{E}-03$ & 12.2 & 52.0 \\
\hline 3 & M46J/TC250 & {$\left[0_{4} / 90_{4}\right]_{\mathrm{s}}$} & 0.14 & 4.5 & $9.02 \mathrm{E}-03$ & 15.7 & 58.9 \\
\hline 4 & M46J/TC250 & {$\left[0_{4} / 90_{4}\right]_{\mathrm{s}}$} & 0.14 & 5.5 & $9.02 \mathrm{E}-03$ & 19.2 & 65.2 \\
\hline 5 & M46J/TC250 & {$\left[0_{4} / 90_{4}\right]_{\mathrm{s}}$} & 0.14 & 8.0 & $9.02 \mathrm{E}-03$ & 27.9 & 78.6 \\
\hline 6 & $\begin{array}{c}\text { S-2 } \\
\text { Glass/epoxy }\end{array}$ & {$\left[0_{10} / 90_{10}\right]$} & 0.11 & 9.3 & $9.02 \mathrm{E}-03$ & 32.4 & 84.7 \\
\hline
\end{tabular}

In order to determine the force-time profile of the impact event for comparison with the finite element model, an accelerometer was attached to the impactor carriage. The accelerometer is by PCB Piezotronics Inc. The raw voltage signal from the accelerometer was conditioned using a PCB Piezotronics Model 48A22 signal 
conditioner. An LDS Dactron data acquisition system was used to capture the signal and provide the appropriate calibrated gain of $10.89 \mathrm{mV} / \mathrm{g}$.

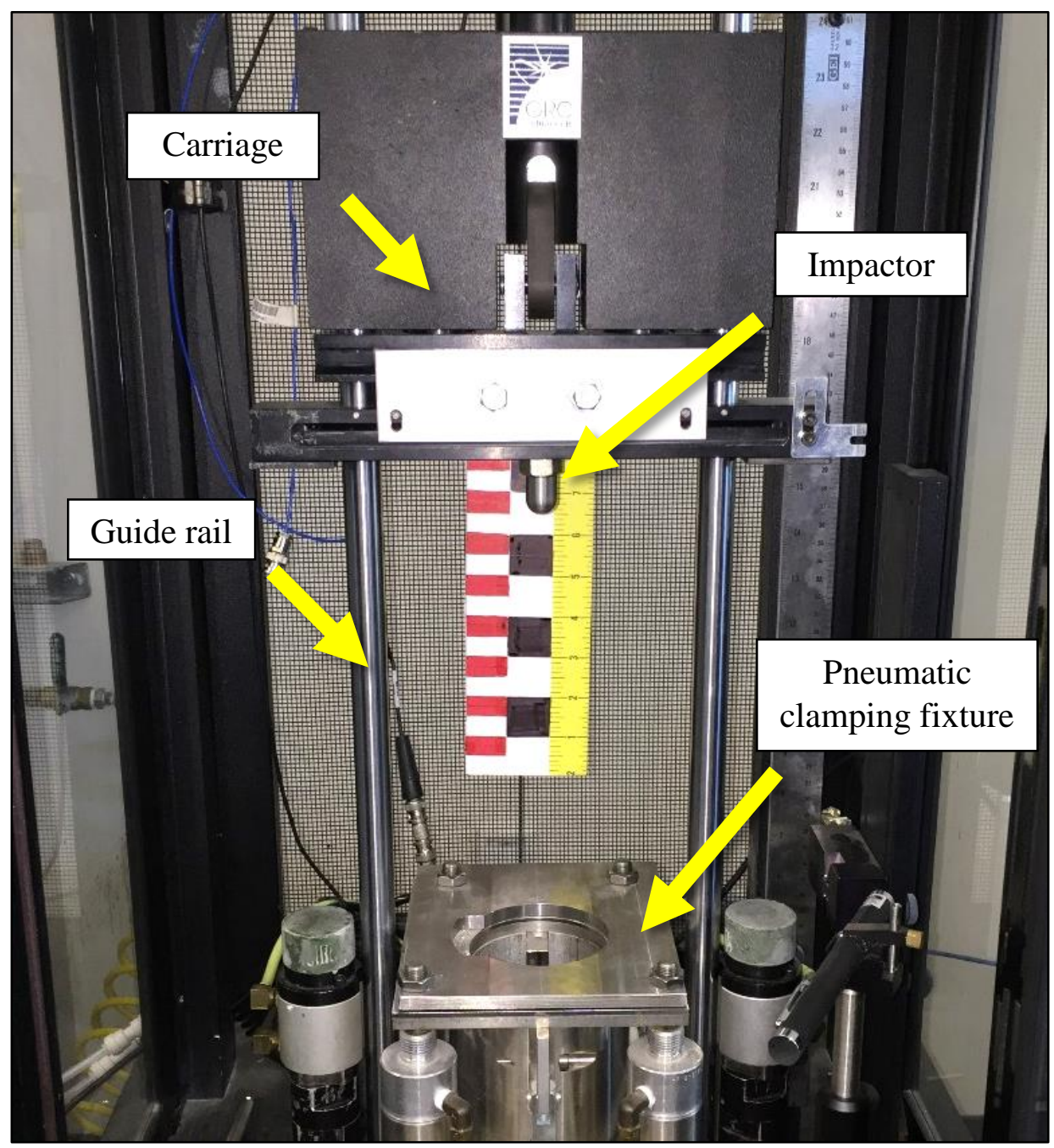

Figure 34 Instron Dynatup 8250 drop weight impact machine

The clamping fixture is pneumatically controlled with a maximum pressure of 95 psi. Unfortunately, the top and bottom plates used for clamping the specimen are not symmetric. The bottom plate has a three inch diameter circle cutout while the top plate 
has the same cutout but with an extra cutout section on one side for instrumentation wiring such as a strain gage. This extra cutout in the top clamping plate is shown in Figure 35. Due to meshing concerns and the relatively low velocity of the impact event, it was decided that the finite element model would not contain the asymmetric cutout. Instead, the symmetric mesh shown in Section 2.3 was created and a clamped boundary condition was created around the outer edge of the plate. Early meshing strategies resulted in elements with poor aspect ratio or unreasonably large numbers of elements for computational efficiency.

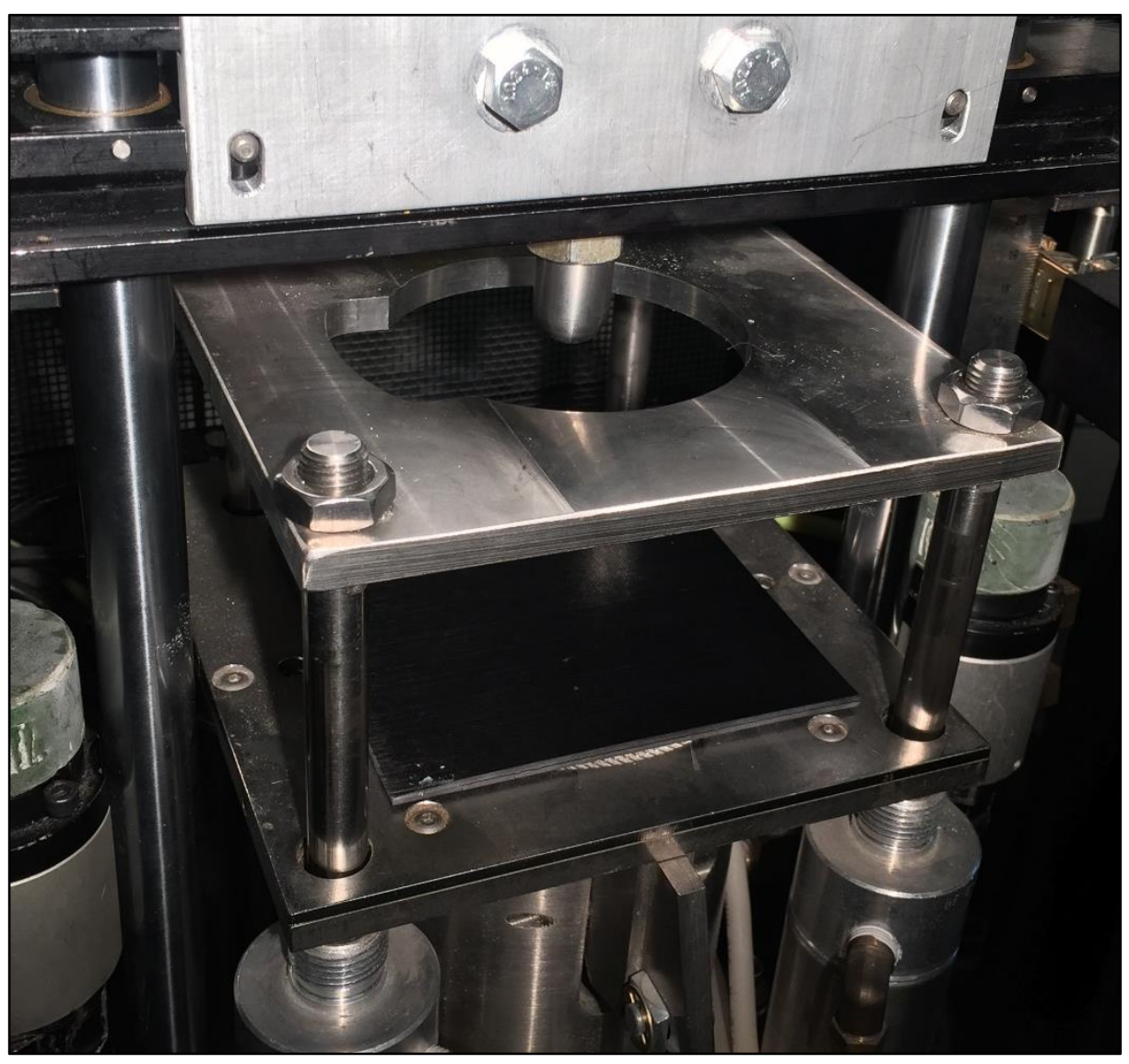

Figure 35 Dynatup 8250 clamping fixture 
The impact event was captured using a high-speed video camera to ensure that the impact occurred as expected and to determine the impactor velocity immediately prior to impact. However, the calculated impactor velocity using the high-speed video footage was found to be inaccurate and is discussed in Section 3.3.

\subsection{Impact Testing Results}

The recorded acceleration-time profiles were post-processed using Matlab ${ }^{\circledR}$ to determine the contact force between the laminate and impactor during the impact event. Figure 36 - Figure 39 show the resulting unfiltered acceleration curves for several different impact energies. While the impact velocity and corresponding impact energy would ideally be computed based on measured velocity of the impactor the moment before impact, the values given in Table 6 were computed using basic kinematic equations and the potential energy prior to dropping the impactor. This was done due to unreliable high-speed camera velocity calculations. Losses due to bearing friction and other dissipating sources are assumed to be small. 


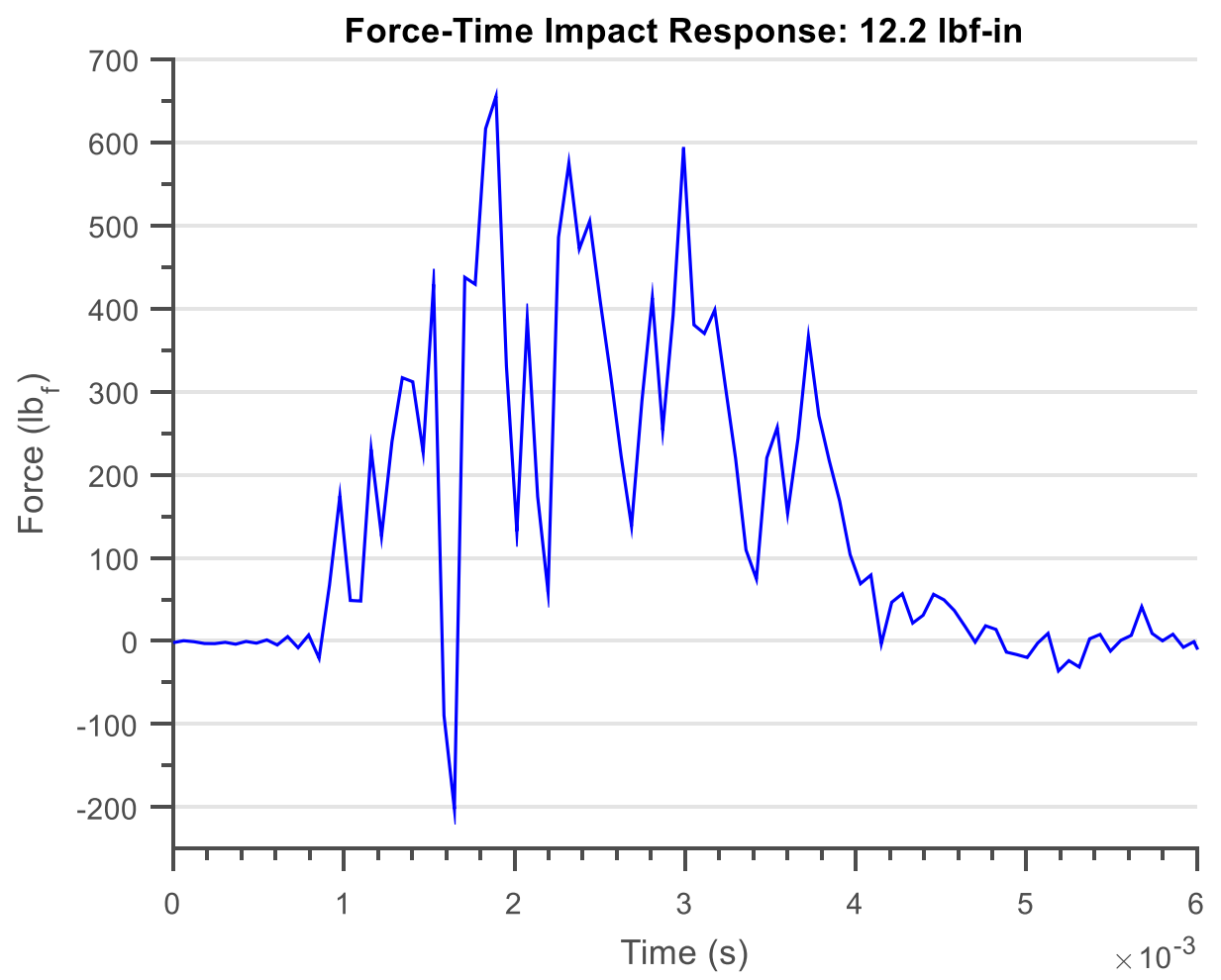

Figure 36 Contact force: $12.2 \mathrm{lb}_{\mathrm{f}}$-in impact energy on $\left[0_{4} / 90_{4}\right]_{\mathrm{s}} \mathrm{M} 46 \mathrm{~J} / \mathrm{TC} 250$ laminate

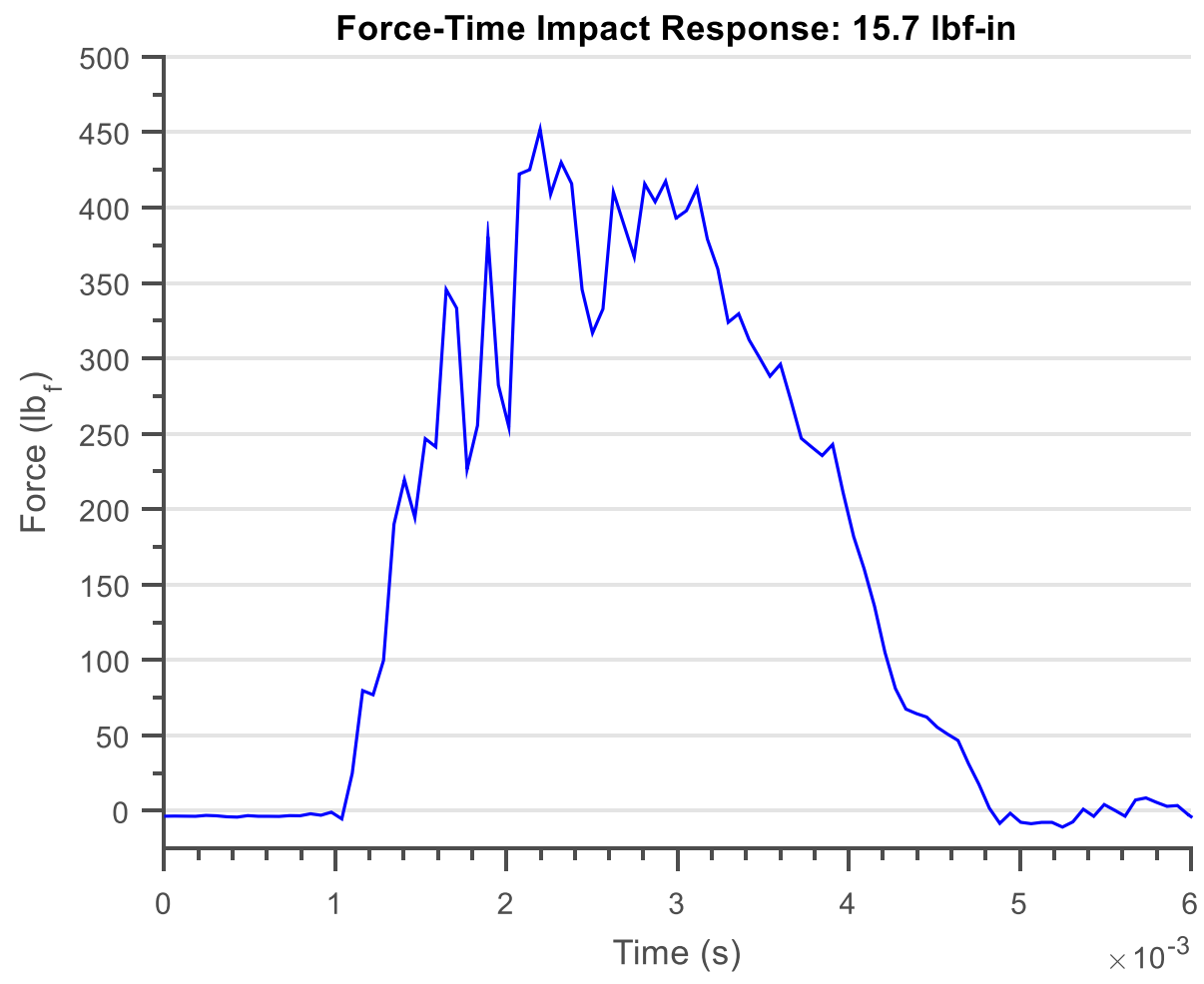

Figure 37 Contact force: $15.7 \mathrm{lb}_{\mathrm{f}}$-in impact energy on $\left[00_{4} / 90_{4}\right]_{\mathrm{s}} \mathrm{M} 46 \mathrm{~J} / \mathrm{TC} 250$ laminate 


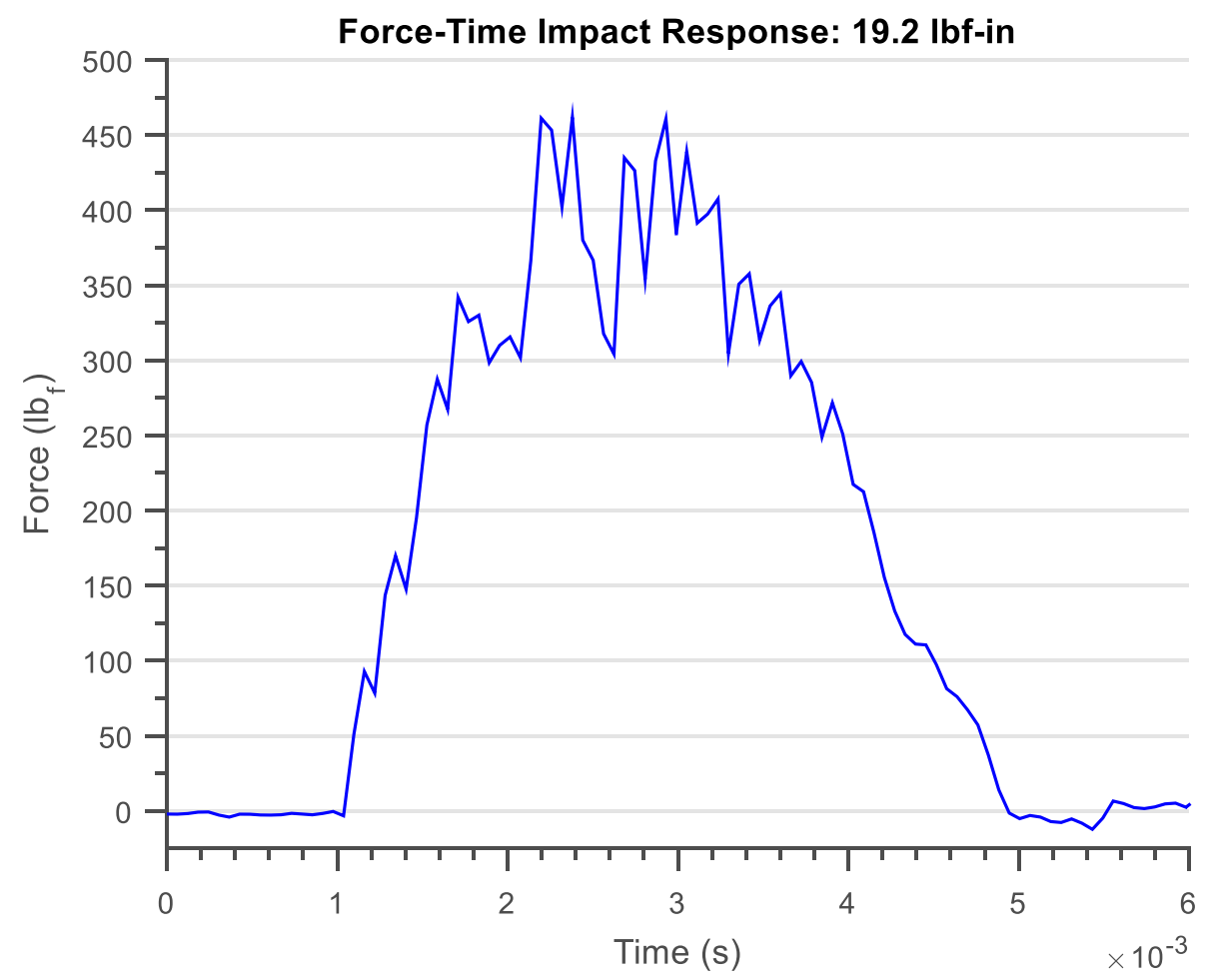

Figure 38 Contact force: $19.2 \mathrm{lb}_{\mathrm{f}}$-in impact energy on $\left[0_{4} / 90_{4}\right]_{\mathrm{s}} \mathrm{M} 46 \mathrm{~J} / \mathrm{TC} 250$ laminate

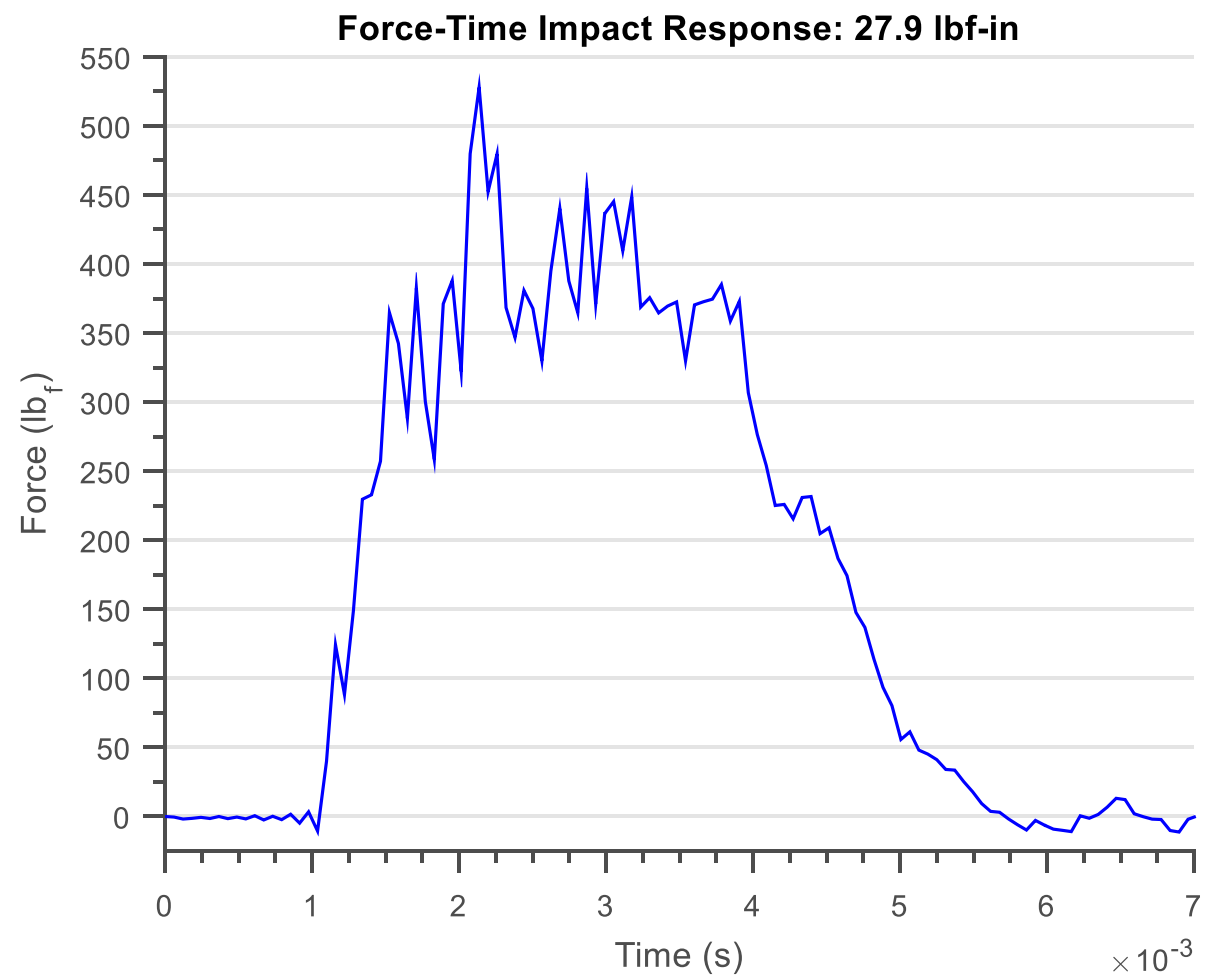

Figure 39 Contact force: $27.9 \mathrm{lb}_{\mathrm{f}}$-in impact energy on [0/4/90 $]_{\mathrm{s}} \mathrm{M} 46 \mathrm{~J} / \mathrm{TC} 250$ laminate 


\section{CHAPTER 4: VIBROTHERMOGRAPHY EXPERIMENTAL TESTING}

\subsection{Introduction}

Glass/epoxy composite laminates are translucent in nature and damage between plies throughout the thickness of the part can be seen under bright lighting of the specimen. An example of this is shown in Figure 40. This S-2 glass/epoxy $\left[0_{10} / 90_{10}\right]$ laminate was impacted with 32.4 lbf-in of impact energy. Carbon fiber reinforced composites, however, are opaque in appearance and damage between the outermost plies is not readily visible. One technique to examine this unseen damage is to vibrate the specimen at a range of frequencies and look for the heat generation created by defects such as cracks, disbands, or delaminations. This nondestructive technique requires an infrared camera and excitation system to vibrate the specimen, as described by Renshaw [33].

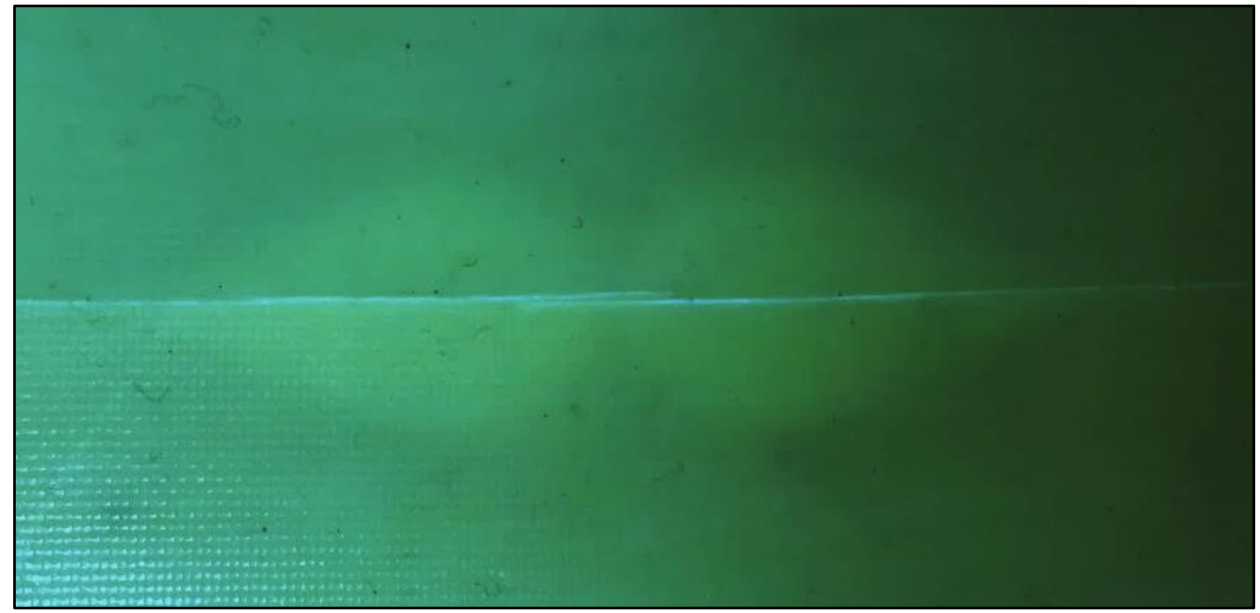

Figure $40 \mathrm{~S}-2$ glass/epoxy $\left[0_{10} / 90_{10}\right]$ laminate, 32.4 lbf-in impact energy 


\subsection{Vibrothermography Experimental Test Set Up}

The experimental setup used to evaluate the damage present in the post-impact composite panels is very similar to the method described by Renshaw [33]. The test setup is shown in Figure 41. The impacted specimen was clamped and vibrated using a broadband piezoelectric excitation system with typical vibration frequencies between 20 $40 \mathrm{kHz}$. A clamped specimen is shown in Figure 42. A pneumatic linear actuator was used to clamp the specimen between the pneumatic transducer and the fixture with 13 psi. The clamping method and fixturing to hold the panel in place during vibration was not as consistent as was desired, but the resulting damage evaluation did not seem to be greatly affected. Some specimens shifted during vibration due to clamping the specimen on only one end.

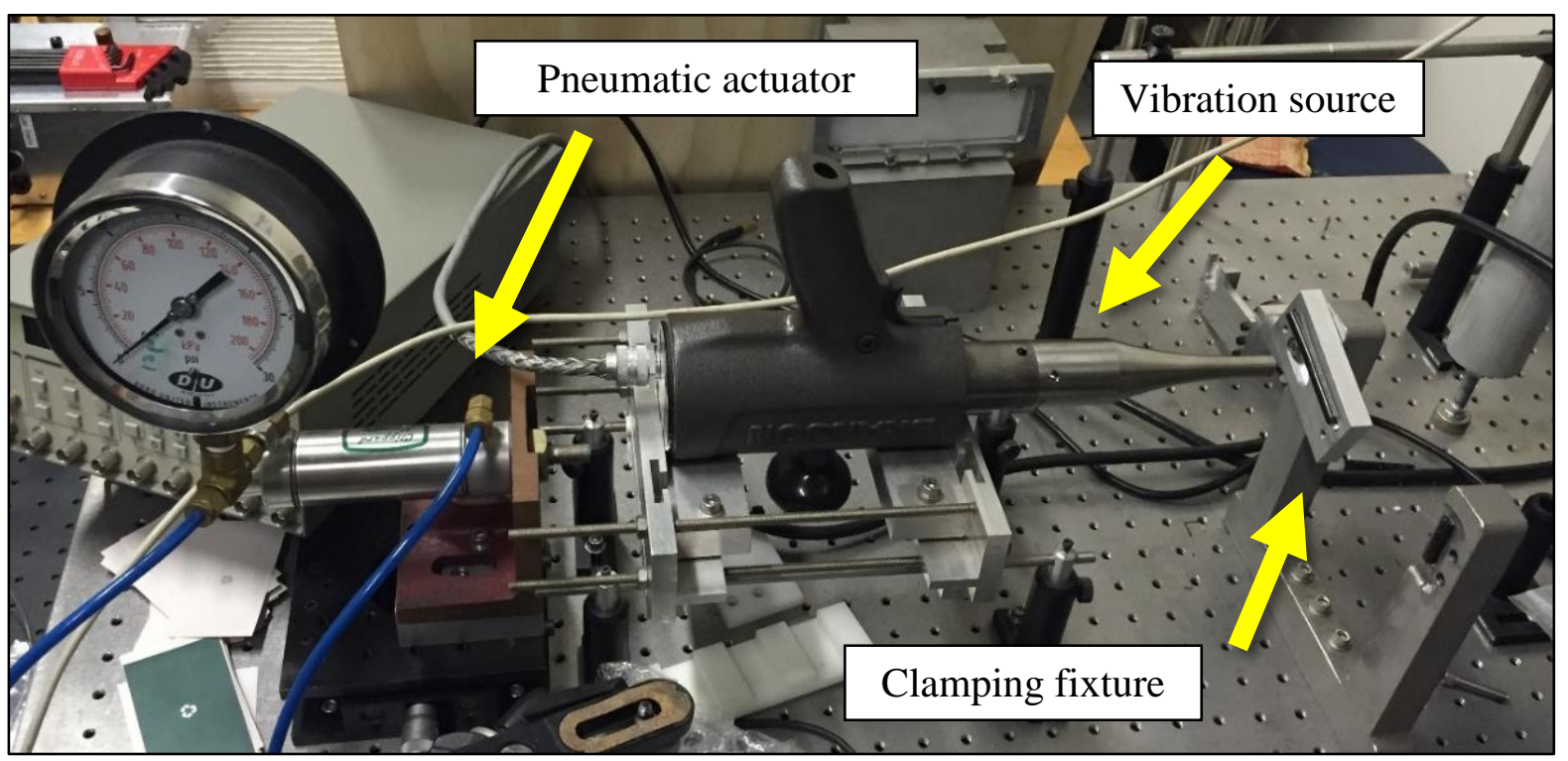

Figure 41 Experimental setup for exciting specimen and viewing heat generation 


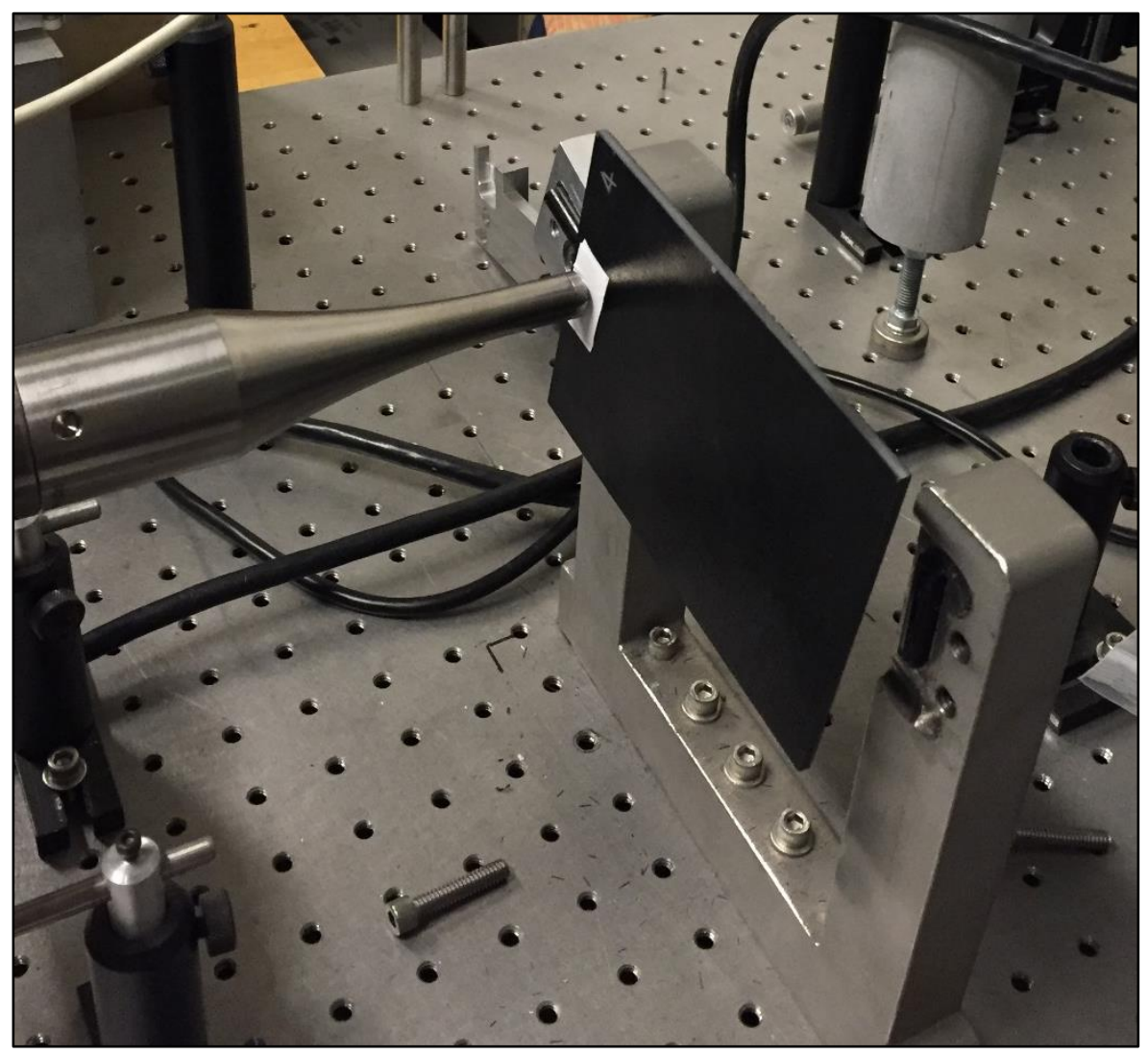

Figure 42 Clamped $\left[0_{4} / 90_{8} / 0_{4}\right]$ composite specimen

Ideally, the specimen would be supported on both ends to ensure consistent vibration from specimen to specimen. Previous experiments using this technique have shown that the stresses induced by vibration do not significantly damage the material further, but that cannot be independently verified from this experiment. An infrared camera measures the heating at the surface of the laminate during vibration. Images are recorded and the transient relative temperature distribution can be viewed.

\subsection{Vibrothermography Results}

Vibrothermography confirmed the expected general shape and location of the delaminations in a $\left[0_{4} / 90_{8} / 0_{4}\right]$ laminate subjected to low-velocity impact from the 
literature. While the vibrothermography technique is a powerful NDT method, there are some characteristics of the technique that must be understood for a correct interpretation of the results. The heat generation at damaged locations in the laminate gives an image of where damage has occurred, but the effects of heat conduction and the orthotropic nature of the laminate must be considered. For example, the first damaged portion of the laminate that is readily apparent when viewing the recorded thermal images is the surface level damage. This is defined as the impacted surface in Figure 43. Prior to vibrothermographic analysis, the impacted side of the laminate visually shows small

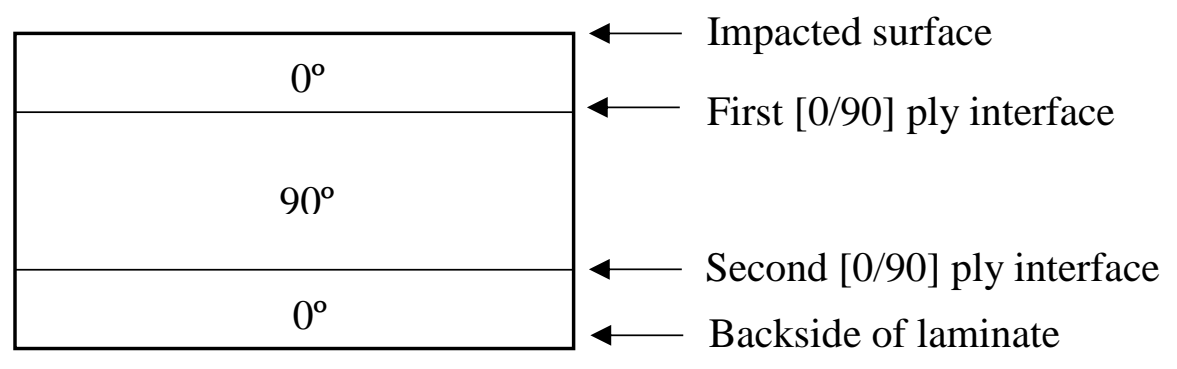

Figure 43 Stacking sequence for impacted laminate tested using vibrothermography signs of local indentation and cracking from contact with the impactor. During vibrothermography, this damage appears as a local region of heat general. Over time, the heat generated from the surface damage diffuses through the laminate. This transient conduction gives the sense that the damage is becoming larger during vibrothermography but in reality, the heat is merely diffusing away from the location where it was generated.

Just as the constitutive mechanical properties of a composite laminate are orthotropic, the thermal conductivity is directionally dependent. In general, carbon fibers conduct heat much better than resin systems. Because of this, asymmetric conduction occurs with heat diffusing along the fiber direction in each ply much faster than through 
the resin. This made it slightly more difficult to discern where there is truly damage in the specimen.

The first $[0 / 90]$ ply interface in the $\left[0_{4} / 90_{8} / 0_{4}\right]$ laminate is the predicted location of the first major delamination, with the next [90/0] ply interface being the location of the second major delamination. These locations are shown in Figure 43 and in the diagram of Figure 44. Since these major delaminations are not present at the surface, it takes time for the heat generated due to frictional rubbing between plies to diffuse through the laminate and up to the imaging surface viewed by the infrared camera. Another issue this creates is that the heat from the local surface damage is still present by the time the heat from the interior delaminations reaches the impacted surface of the laminate. Since the infrared camera uses software that contours the image based on the relative temperatures in the image, the local damage dominates by showing up much brighter than the damage at the first [0/90] ply interface. Figure 45 shows this effect from a tested specimen. While the heat signature generated by the peanut-shaped delamination appears faint after diffusing through four plies, it is clear that there is damage present at the [0/90] interface below the surface. Appendix E contains all of the experimental infrared images captured using vibrothermography. 

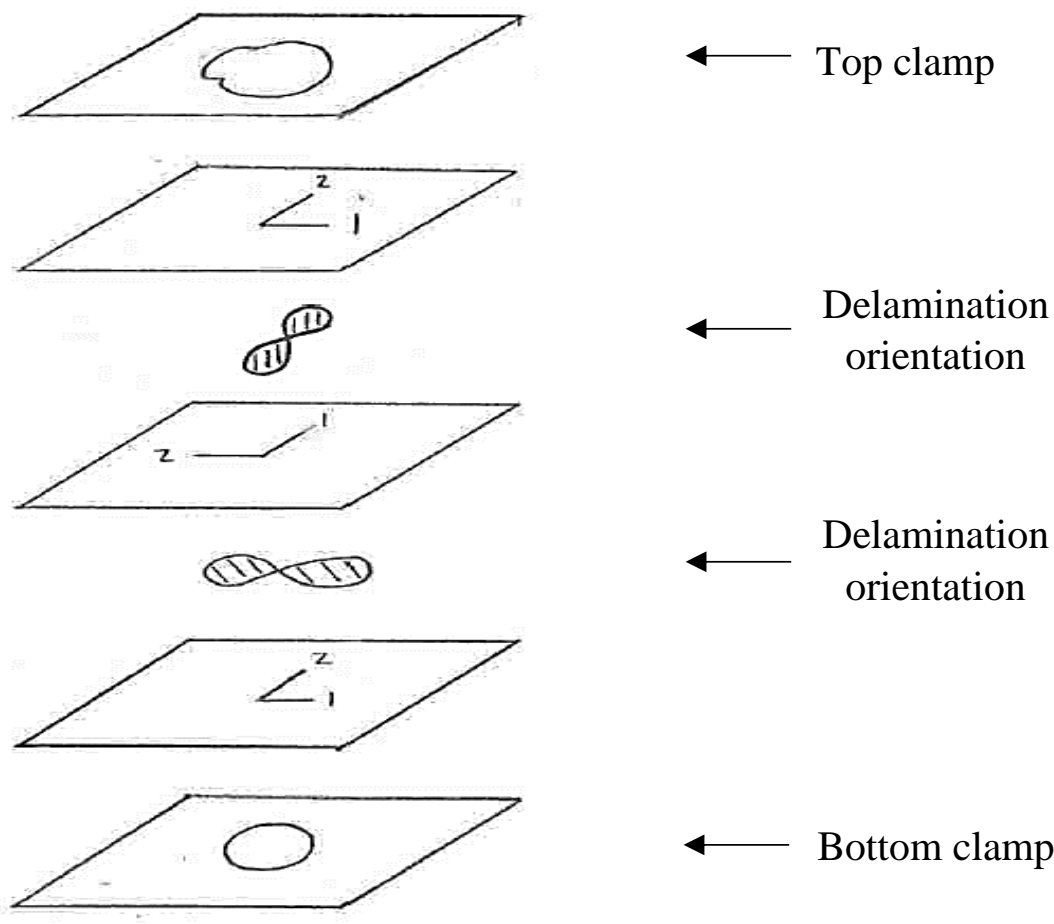

Figure 44 Clamped specimen stacking sequence with predicted delamination shapes

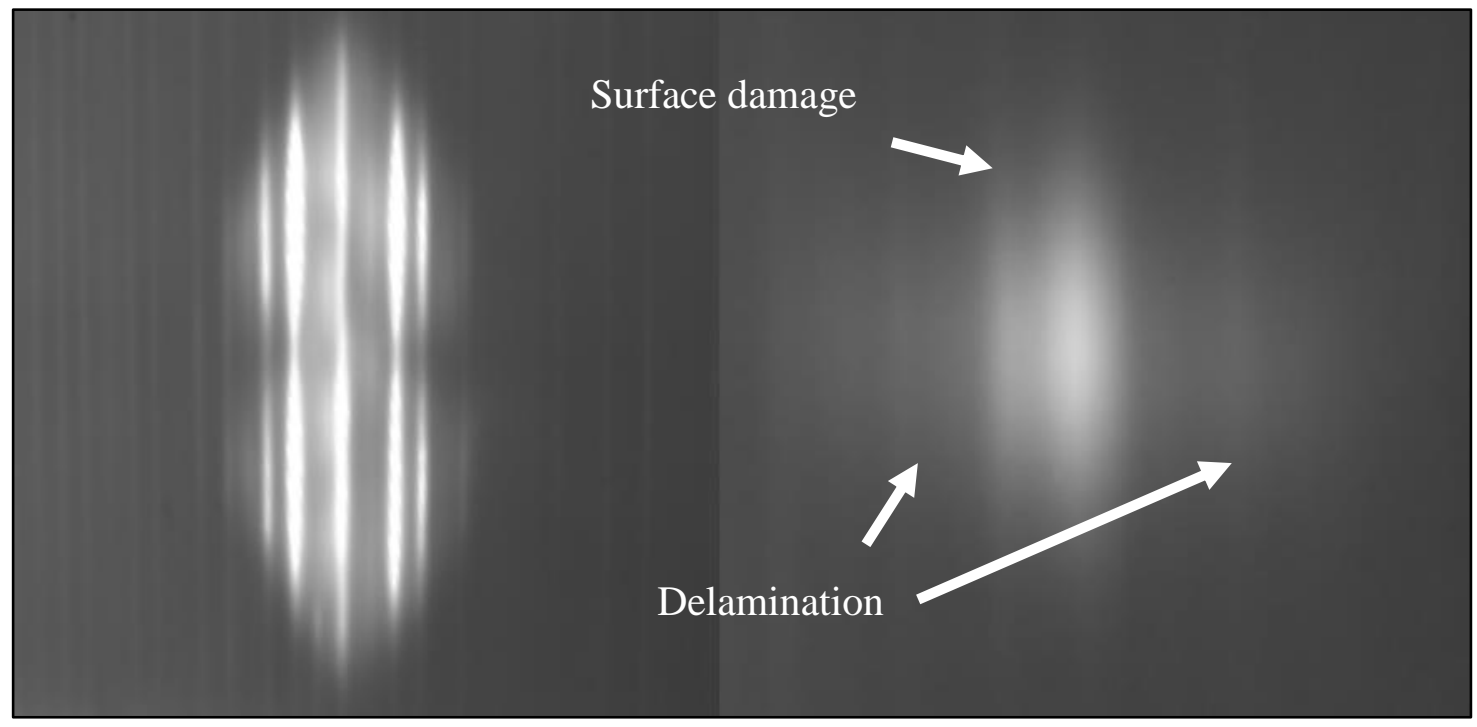

Figure 45 Vibrothermography IR images: backside view (left) and impact side (right) 


\section{CHAPTER 5: CONCLUSIONS}

The goal of this research was to confirm the validity of flexural stiffness-based methods for predicting the relative size and shape of delamination damage due to a lowvelocity impact event. In addition, the contact tiebreak method in LS-DYNA ${ }^{\circledR}$ was used to predict interlaminar ply delamination at sublaminate interfaces. Vibrothermography and impact testing was performed to ground the finite element simulations and analytical predictions in physical results.

Mode I fracture simulations were a good starting point to tune the parameters of the contact tiebreak definition and ensure that the results were reasonable. It was found that mesh dependency and computational limitations were the most difficult part of the simulation. Peak traction stresses were adjusted based on the mesh size until the results of the simulation appeared physically accurate.

With reasonable simple Mode I parameters determined, a full-scale low-velocity impact simulation on a composite laminate was performed. The contact tiebreak definition in LS-DYNA ${ }^{\circledR}$ was able to reasonably predict a portion of the delamination size and shape when compared to physical testing. Discrepancies in simulation results and physical experiments are likely to due to assumed material elastic and fracture properties. Additionally, the omission of failure modes other than delamination is not able to completely capture delamination. The interaction between failure modes is complex and model this interaction is necessary for further accuracy to be achieved. The force vs. time prediction of the finite element simulation achieved good agreement with experimental data. A small difference in the contact time of the impact event is likely due to reduced compliance from the omission of other failure modes. A less compliant laminate results 
in a shorter contact time, and the inclusion of other failure modes would reduce the stiffness of the laminate.

Impact testing on carbon fiber composite laminates under low-velocity impact results in interplay delamination failure that is not visible through the opaque laminate. Vibrothermography successfully revealed the locations and relative size of delamination damage. It is noted that diffusion properties of the orthotropic laminate must be considered in order to understand the infrared images seen in vibrothermography testing. Heat diffusion through conduction between plies in the laminate is limited and results in the appearance of only a portion of the laminate's damage. 


\section{CHAPTER 6: FUTURE RECOMMENDATIONS}

1. Isolation of delamination failure mode by very small drop heights where only delamination damage occurs will help reduce the effect of failure mode interaction. This would lead to a more accurate prediction of the force vs. time response of the impact event for an experimental impact.

2. Additional material fracture properties would help ensure that the delamination initiates and propagates in a realistic fashion and eliminate some of the spurious damage seen in the finite element simulation. While an iterative trial and error approach was used in this research, a study using a systematic approach could also help determine the effect of each contact tiebreak parameter in the resulting delamination process.

3. Vibrothermography was shown to be a good technique for non-destructive evaluation of the internal delamination damage. Future investigation to isolate the effects of the heat diffusion process using simple impact tests on simple laminates would be very informative. Varying ply thicknesses could be used to calibrate the damage from the infrared images and analyze the transient heat conduction process through the thickness of the laminate. In addition, a coupled thermal-mechanical finite element model could simulate the frictional heating due to delamination damage for comparison to physical testing. 


\section{BIBLIOGRAPHY}

[1] A. L. Stratton and A. A. Pelegri, "Investigation of interlayer and intralayer delaminations," 1999. [Online]. Available: http://rutgersscholar.rutgers.edu/volume01/pelestra/pelestra.htm.

[2] B. D. Agarwal, L. J. Broutman and K. Chandrashekhara, Analysis and Performance of Fiber Composites, 3rd Edition, Hoboken, New Jersey: Wiley, 2006.

[3] G. T. Mase and G. E. Mase, Continuum Mechanics for Engineers, Boca Raton: CRC Press, 1999.

[4] D. Liu, "Impact-Induced Delamination - A View of Bending Stiffness Mismatching," Journal of Composite Materials, vol. 22, pp. 674-691, July 1988.

[5] S. R. Finn and G. S. Springer, Composite Plates Impact Damage An Atlas, Lancaster: Technomic Publishing Co., Inc., 1991.

[6] G. A. O. Davies and X. Zhang, "Impact Damage Prediction in Carbon Composite Structures," International Journal of Impact Engineering, vol. 16, no. 1, pp. 149-170, 1995.

[7] M. Liangkai and D. Liu, "Delamination and fiber-bridging damage analysis of angle-ply laminates subjected to transverse loading," Journal of Composite Materials, pp. 1-13, 2015.

[8] S. A. Muflahi, G. Mohamed and S. R. Hallet, "Investigation of Delamination Modeling Capabilities for Thin Composite Structures in LS-DYNA," in 13th International LS-DYNA Users Conference.

[9] O. Shor and R. Vaziri, "Through-Thickness Element Splitting for Simulation of Delamination in Laminated Composite Materials," in 13th International LS-DYNA Users Conference.

[10] S. Abrate, Impact on Composite Structures, Cambridge: Cambridge University Press, 1998.

[11] J. D. Mello, "Automated Data Acquisition and Analysis of Low-Velocity Impact Tests of Composites with Comparisons to Quasi-Static Tests and Analytical Models," San Luis Obispo, California, 1988.

[12] J. E. J. Smith, "MODE I FRACTURE OF EIGHT-HARNESS-SATIN CARBON CLOTH WEAVES FOR," San Luis Obispo, California, 2013.

[13] "Standard Test Method for Mode I Interlaminar Fracture Toughness of Unidirectional FiberReinforced Polymer Matrix Composites," ASTM D5528-13, West Conshohocken, PA, 2013.

[14] "Standard Test Method for Determination of the Mode II Interlaminar Fracture Toughness of Unidirectional Fiber-Reinforced Polymer Matrix Composites," ASTM D7905/D7905-14, West Conshohocken, PA, 2014.

[15] "Standard Test Method for Mixed Mode I-Mode II Interlaminar Fracture Toughness of Unidirectional Fiber Reinforced Polymer Matrix Composites," ASTM D6671 / D6671M-13e1, 
West Conshohocken, PA, 2013.

[16] R. Borg, L. Nilsson and K. Simonsson, "Simulation of low velocity impact on fiber laminates using a cohesive zone based delamination model," Composites Science and Technology, vol. 64, pp. 279-28, 2004.

[17] Forghani, A. Forghani and e. al., "Modelling of Damage Development in Blast Loaded Composite Panels," in 16th International Conference on Composite Materials, Kyoto, Japan, 2007.

[18] M. Ilyas, C. Espinosa and M. Saulaun, "Dynamic Delamination of Aeronautic Structural Composites by Using Cohesive Finite Elements," in 17th International Conference on Composite Materials, Edinburgh, Scotland, 2009.

[19] G. Kay, "Simulations of Carbon Fiber Composite Delamination Tests," Lawrence Livermore National Laboratory, Livermore, 2007.

[20] M. Loikkanen, G. Praveen and D. Powell, "Simulation of Ballistic Impact on Composite Panels," in 10th International LS-DYNA Users Conference.

[21] LS-DYNA Keyword User's Manual, Livermore, California: Livermore Software Technology Corporation, 2016.

[22] B. Z. Haque and J. W. Gillespie Jr., "Rate Dependent Progressive Composite Damage Modeling using MAT162 in LS-DYNA," in 13th International LS-DYNA USers Conference, Detroit, 2014.

[23] R. Krueger and D. Goetze, "Influence of Finite Element Software on Energy Release Rates Computed Using the Virtual Crack Closure Technique," NASA, Hampton, Virginia, 2006.

[24] LS-DYNA Theory Manual, Livermore, California: Livermore Software Technology Corporation, 2016.

[25] J. Day, "Delamination," Livermore Software Technology Corporation, 2015.

[26] K. Song, C. G. Davila and C. A. Rose, "Guidelines and Parameter Selection for the Simulation of Progressive Delamination," in Abaqus Users' Conference, 2008.

[27] "Damping," LSTC Inc, 2016. [Online]. Available:

http://www.dynasupport.com/howtos/general/damping.

[28] R. Rainsberger, TrueGrid User's Manual, XYZ Scientific Applications, Inc., 2006.

[29] J. D. Garrett, "Experimentation of Mode I \& Mode II Fracture of Uni-directional composites and Finite Element Analysis of Mode I using Cohesive Elements \& Cohesive Contact," Cal Poly, San Luis Obispo, 2016.

[30] Toray Carbon Fibers America, Inc., "Torayca M46J Data Sheet," Santa Ana. 
[31] Tencate Advanced Composites, "TC250 Resin System," Morgan Hill.

[32] eFunda, Inc., "Lamina Principal Directions," [Online]. Available:

http://www.efunda.com/formulae/solid_mechanics/composites/images/Lamina_PrincipalDir.gif.

[33] J. Renshaw, J. C. Chen, S. D. Holland and R. B. thompson, "The Sources of Heat Generation in Vibrothermography," NDT\&E International, vol. 44, pp. 736-739, 2011.

[34] B. D. Davidson and R. A. Schapery, "Effect of Finite Width on Deflection and Energy Release Rate of an Orthotropic Double Cantilever Specimen," Journal of Composite Materials, vol. 22, pp. 640-656, July 1988.

[35] Y. Zhang, P. Zhu and X. Lai, "Finite element analysis of low-velocity impact damage in composite laminated plates," Materials and Design, vol. 27, pp. 513-519, 2004.

[36] J. T. Rappolt, "Analysis of a Carbon Fiber Reinforced Polymer Impact Attenuator for a Formula SAE Vehicle Using Finite Element Analysis," San Luis Obispo, California, 2015.

[37] G. Sih and A. Skudra, "Failure Mechanics of Composites," Handbook of Composites, vol. 3, 1985. 


\section{APPENDICES}

APPENDIX A

\section{Matlab $^{\circledR}$ code for bending stiffness mismatch}

\section{GR 11/19/15}

Modified 5/17/16

Name

Composite Delamination Script

Purpose

Thesis

\section{Description}

Calculate the general shape of delaminations in composite laminates using stiffness mismatch theory given by Liu (1988) Journal of Composite Matl and Referenced in Serge Abrate Impact on Composite Structures.

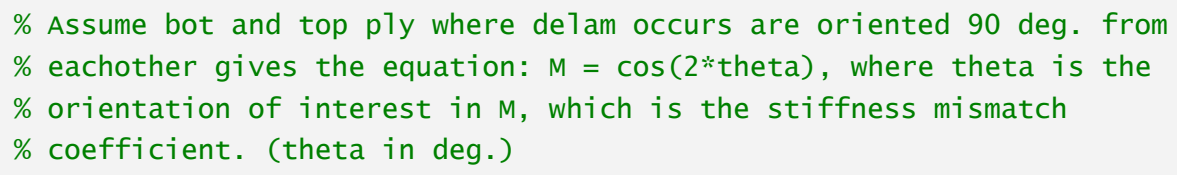

\section{WARNINGS}

Only works for laminates that have two different ply orientations Ex. [90/0],[0_4/90_4], [-15_3/75_3], etc. (as of 11/30/2015)

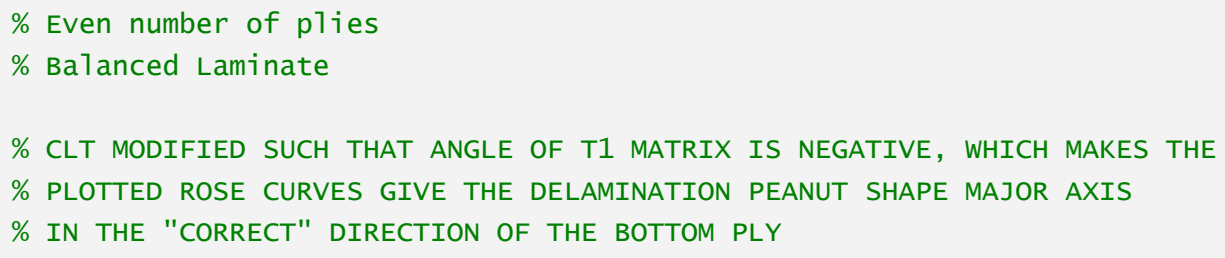

Behavior

close al1; clear;

\section{Inputs}




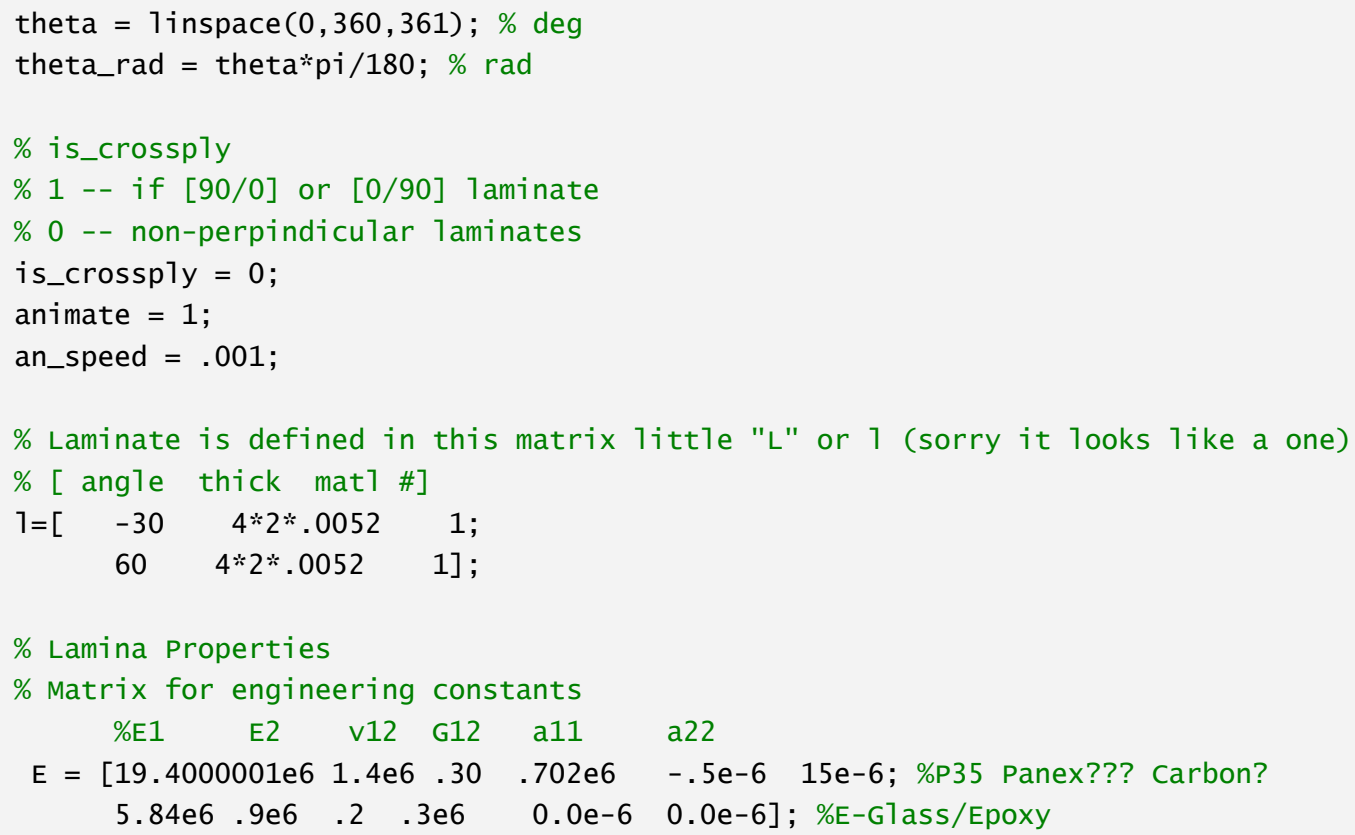

$\% \% \% \% \% \% \% \% \% \% \% \% \% \% \% \% \% \% \% \% \%$ END USER INPUT

$\% \% \% \% \% \% \% \% \% \% \% \% \% \% \% \% \% \% \% \% \% \% \% \% \% \% \% \% \% \% \% \% \% \%$

\section{Script}

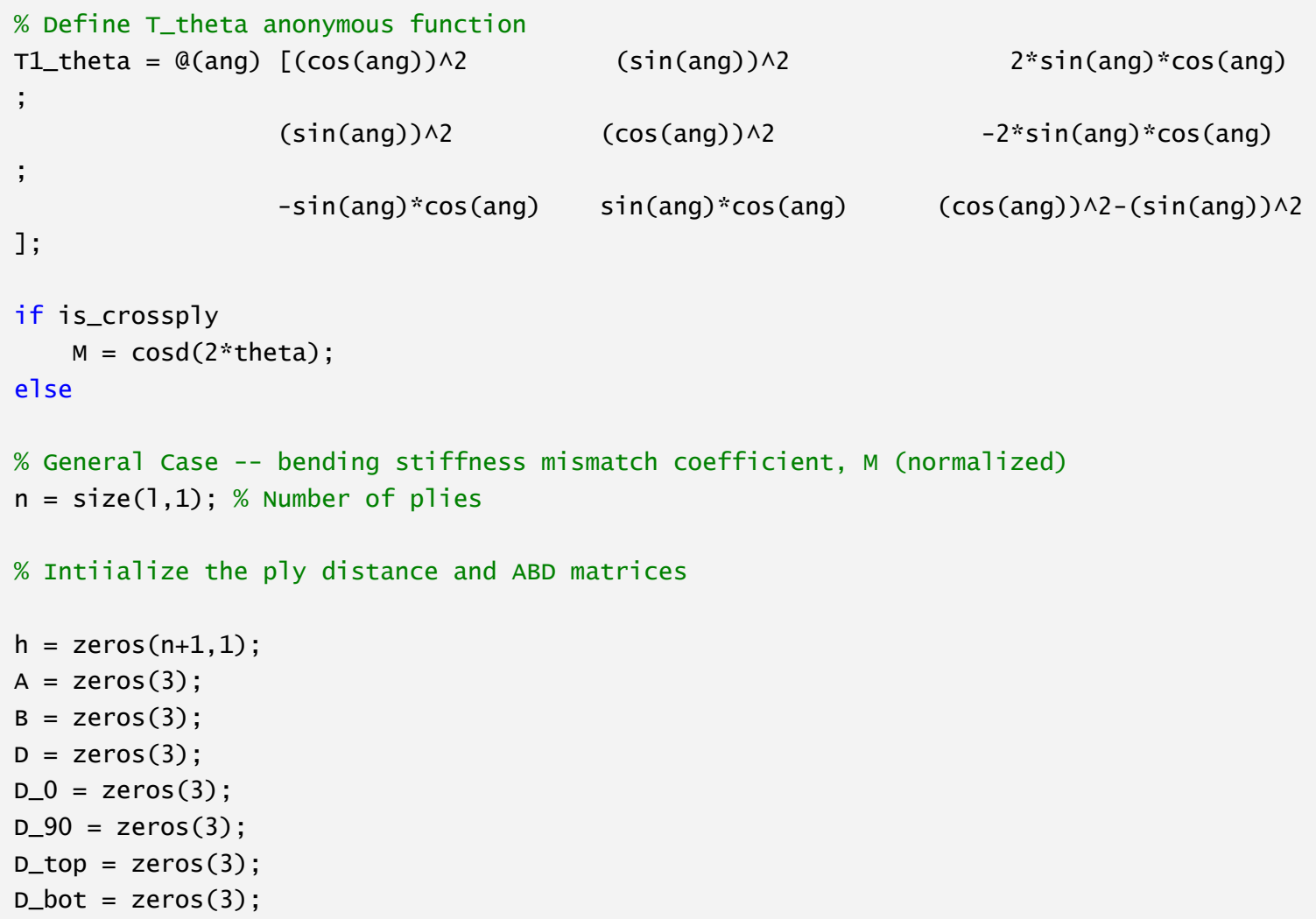




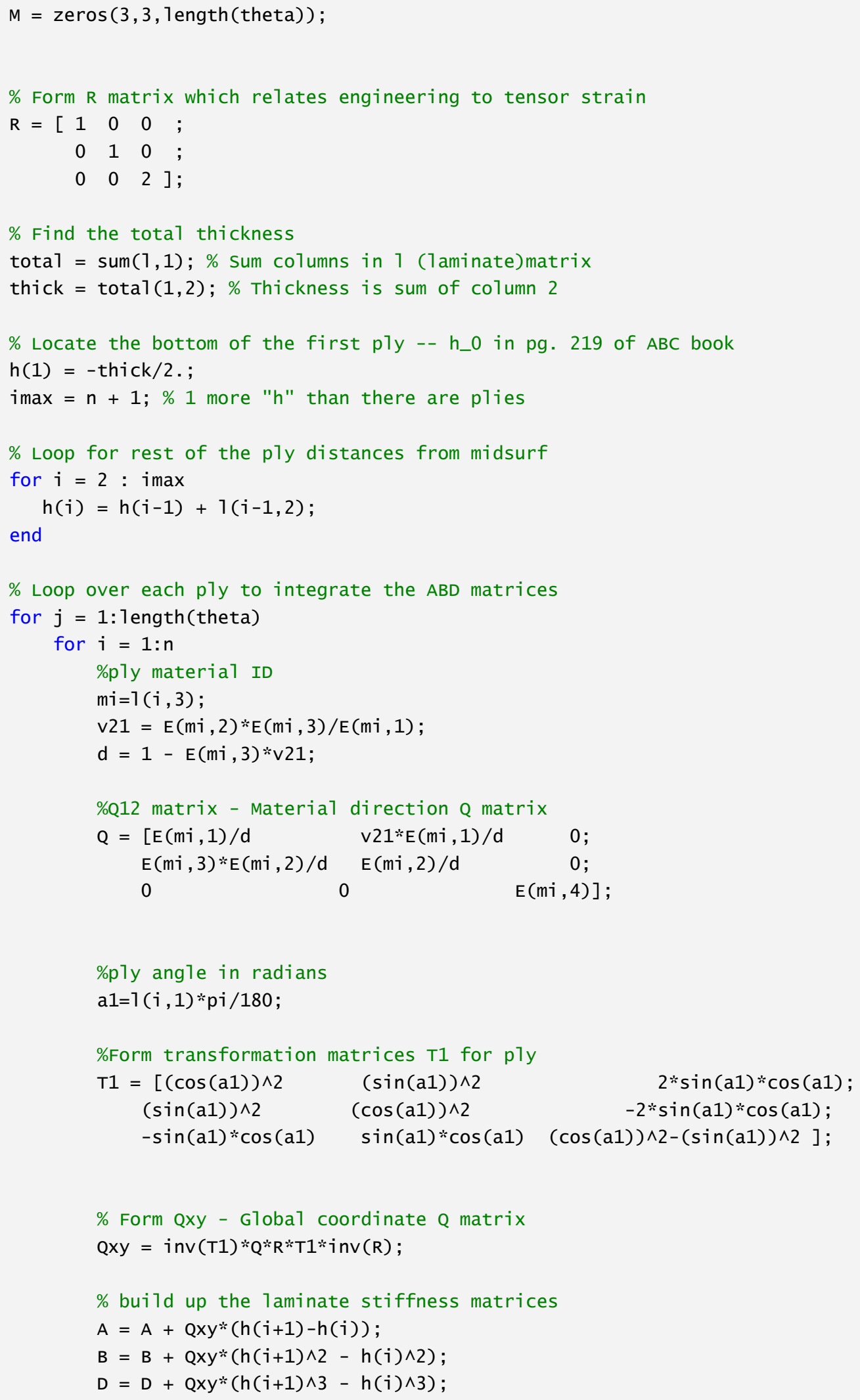


$\% \%$ Ideas :

Figure out how many plies are in "top" and "bot" ply and then use

the number of plies to build a D matrix for the 90 and 0

normalization.

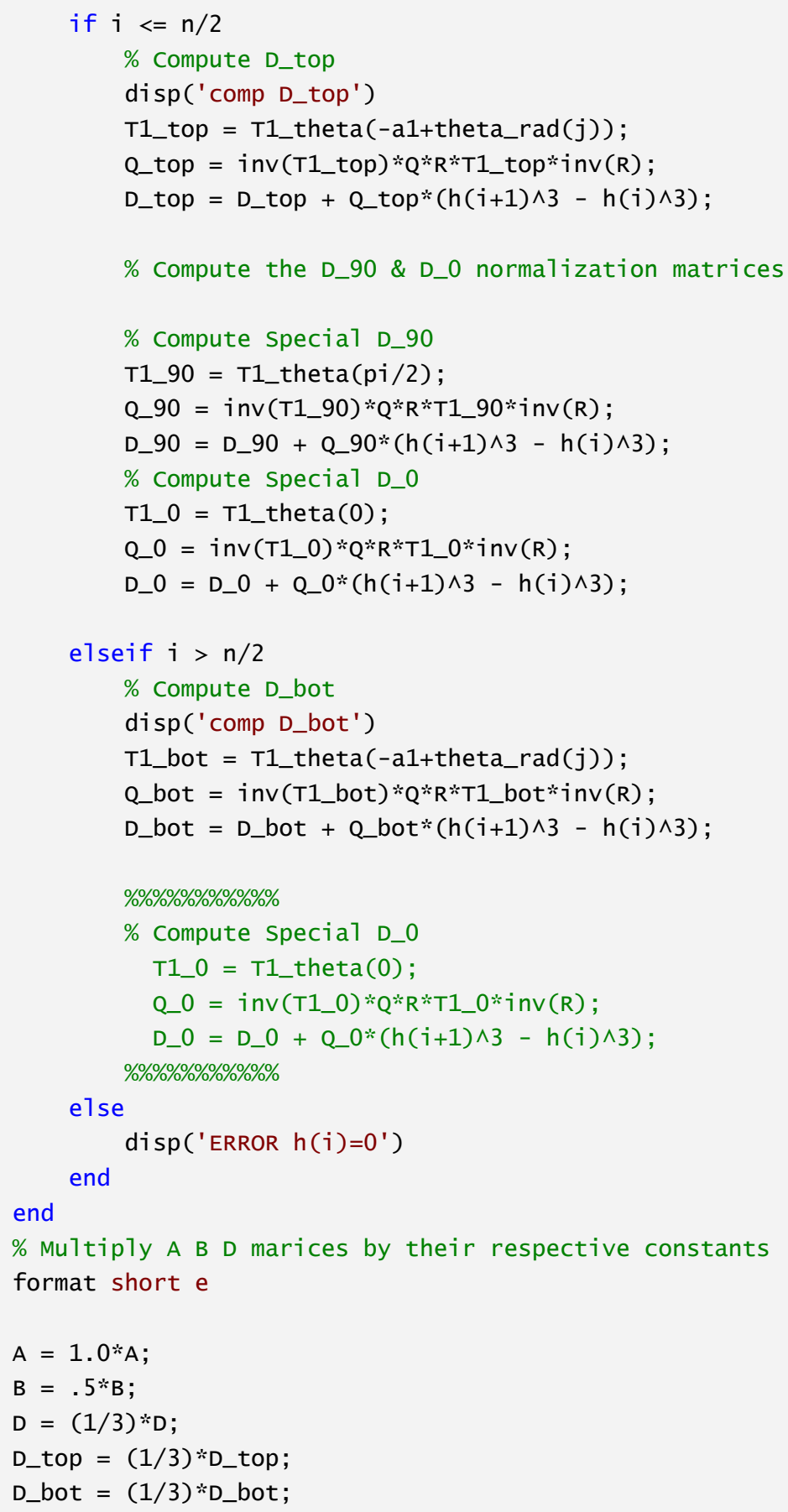




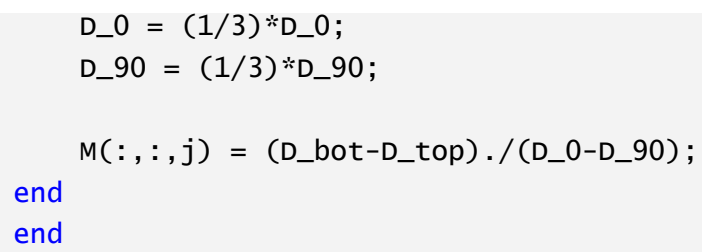

\section{Visualization}

\section{Cross Ply Visualization}

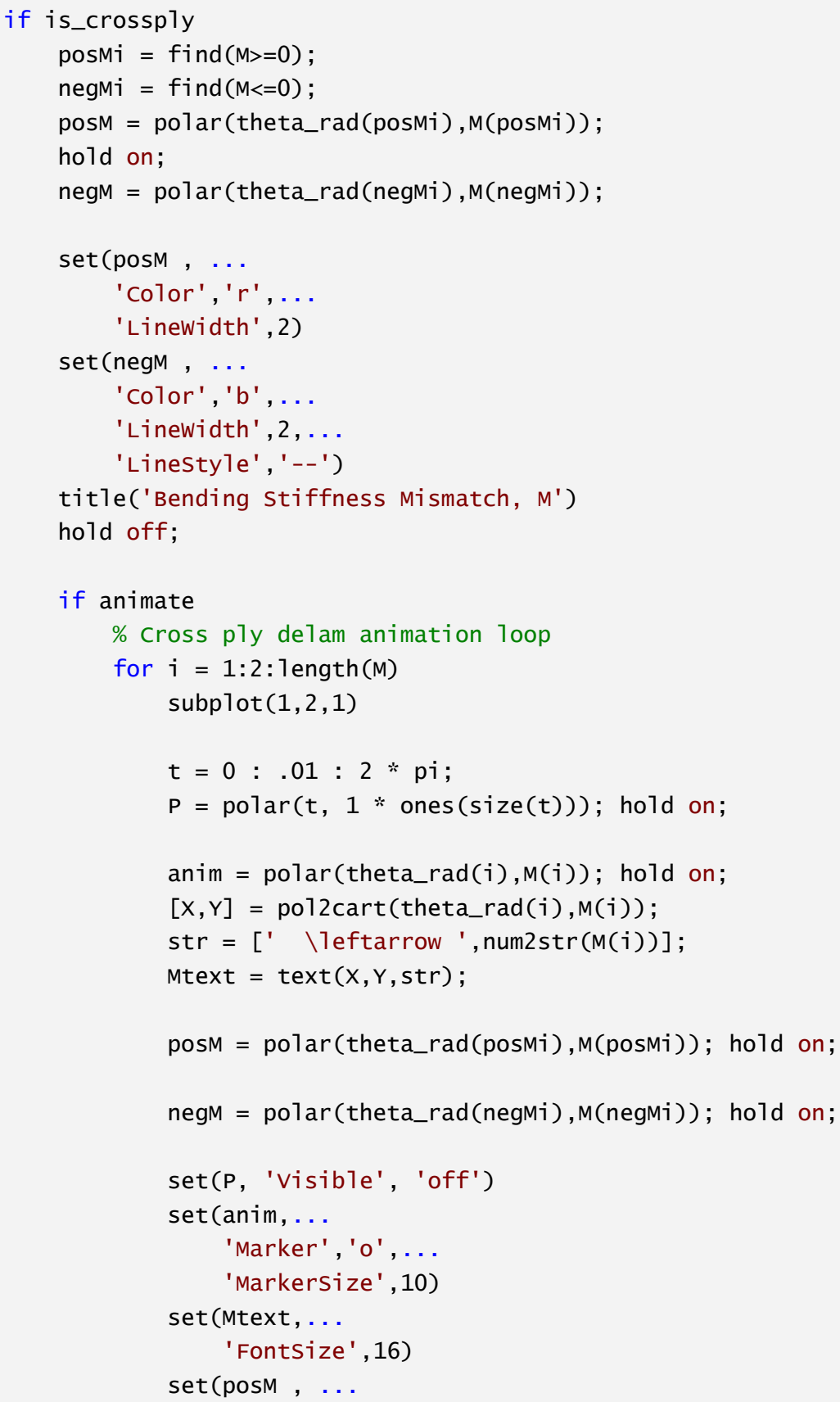




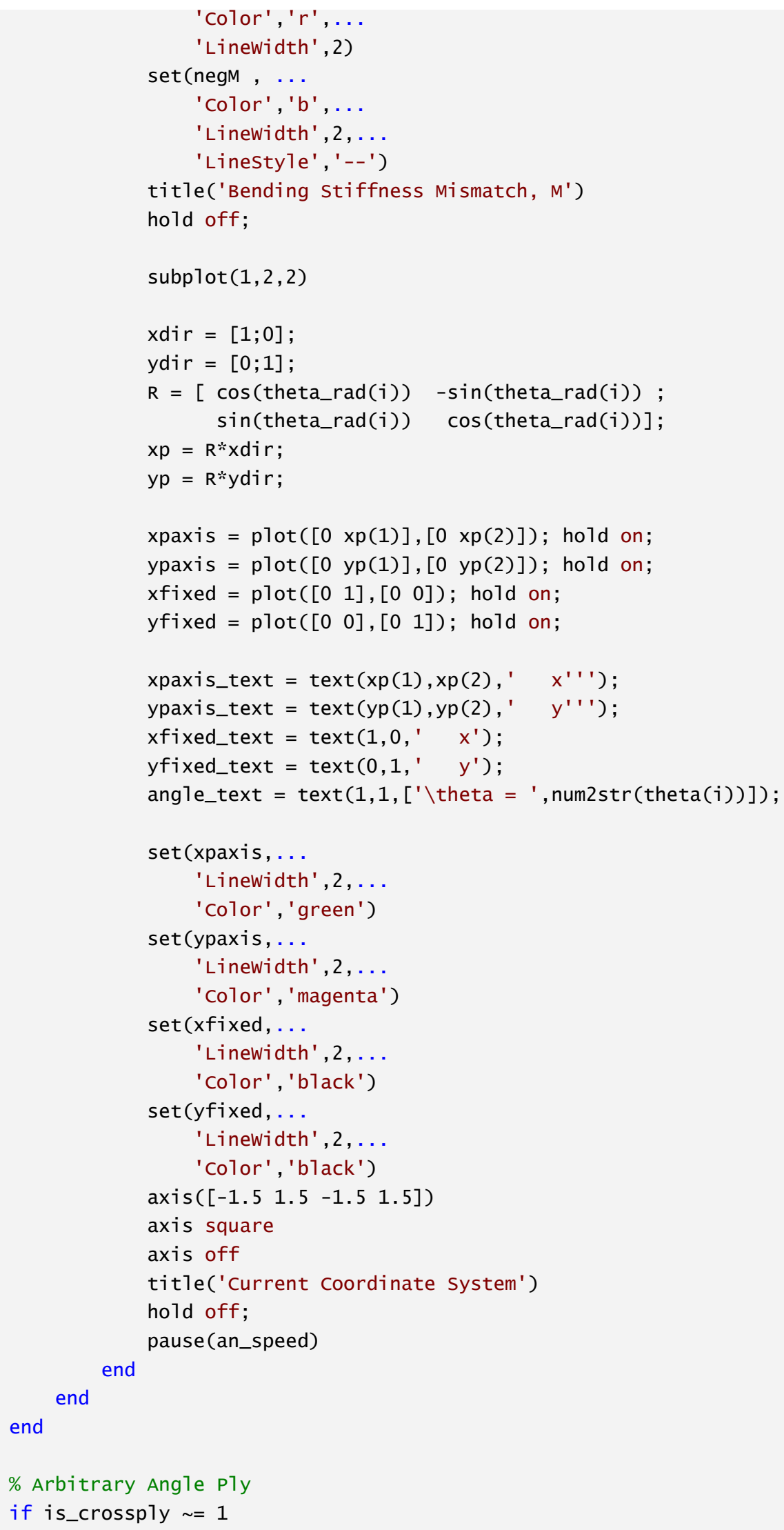



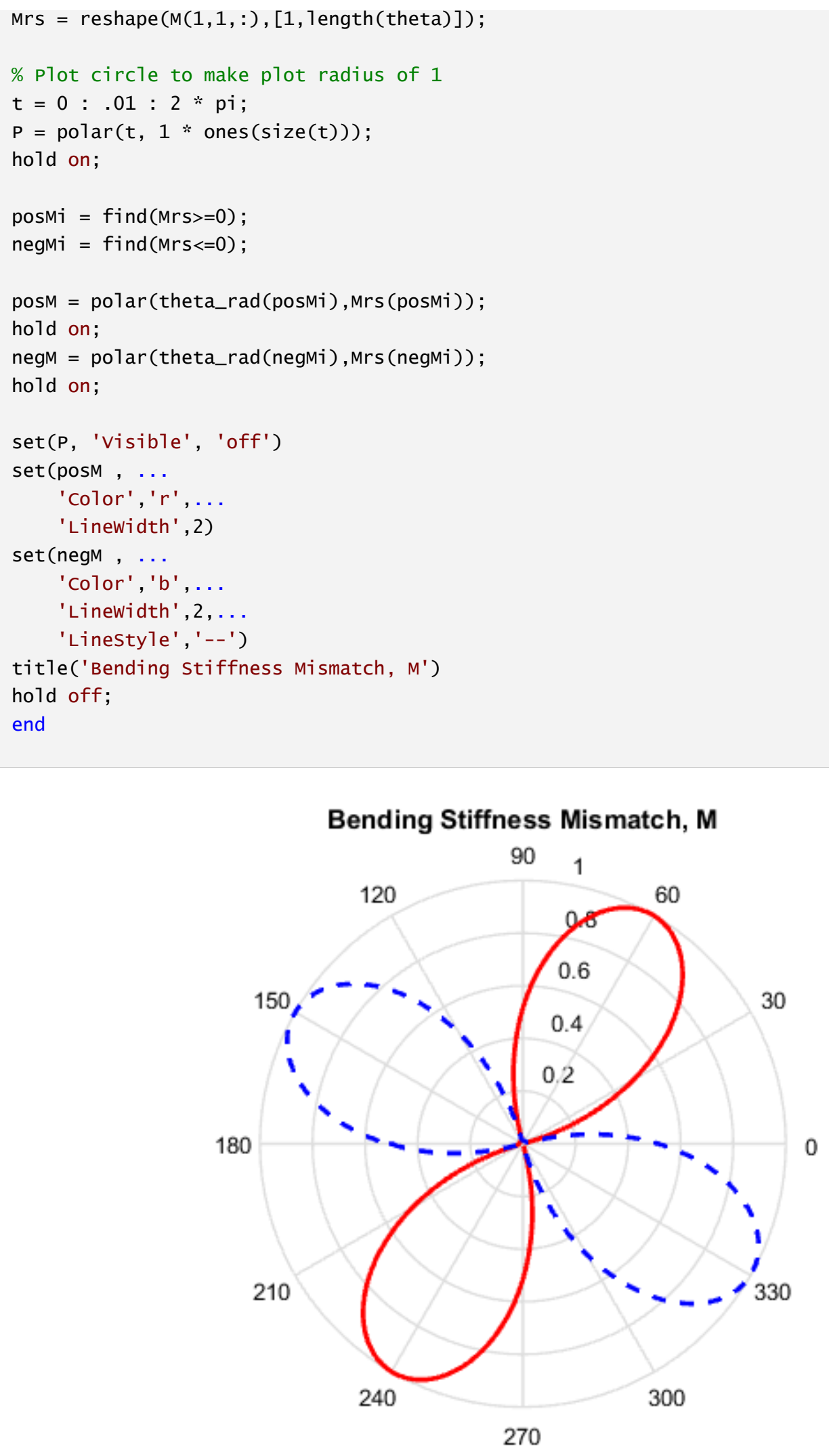
$\underline{\text { Published with MATLAB® R2015a }}$ 


\section{APPENDIX B}

Laminated carbon fiber plate impact reduced keyword file

\$\# LS-DYNA Keyword file created by LS-PrePost 4.0 - 28Jan2013(19:00)

\$\# Created on May-23-2016 (18:27:43)

*KEYWORD

*TITLE

\$\# title

LS-DYNA keyword deck by LS-PrePost

*CONTROL_ACCURACY

\$\# osu inn pidosu

$\begin{array}{lll}0 & 4 & 0\end{array}$

*CONTROL_ENERGY

\$\# hgen rwen slnten rylen

$\begin{array}{llll}2 & 2 & 1 & 1\end{array}$

*CONTROL_TERMINATION

\$\# endtim endcyc dtmin endeng endmas

$\begin{array}{lllll}0.010000 & 0 & 0.000 & 0.000 & 0.000\end{array}$

*CONTROL_TIMESTEP

\$\# dtinit tssfac isdo tslimt dt2ms lctm erode ms1st

$\begin{array}{llllllll}0.000 & 0.800000 & 0 & 0.000 & 0.000 & 0 & 0 & 0\end{array}$

\$\# dt2msf dt2mslc imscl unused unused rmscl

$$
\begin{array}{llll}
0.000 & 0 & 0 & 0.000
\end{array}
$$

*DATABASE_ATDOUT

\$\# dt binary lcur ioopt

3.0000E-6 $0 \quad 0 \quad 1$

*DATABASE_GLSTAT

\$\# dt binary lcur ioopt

1.0000E-5 $0 \quad 0 \quad 0 \quad 1$

*DATABASE_MATSUM 


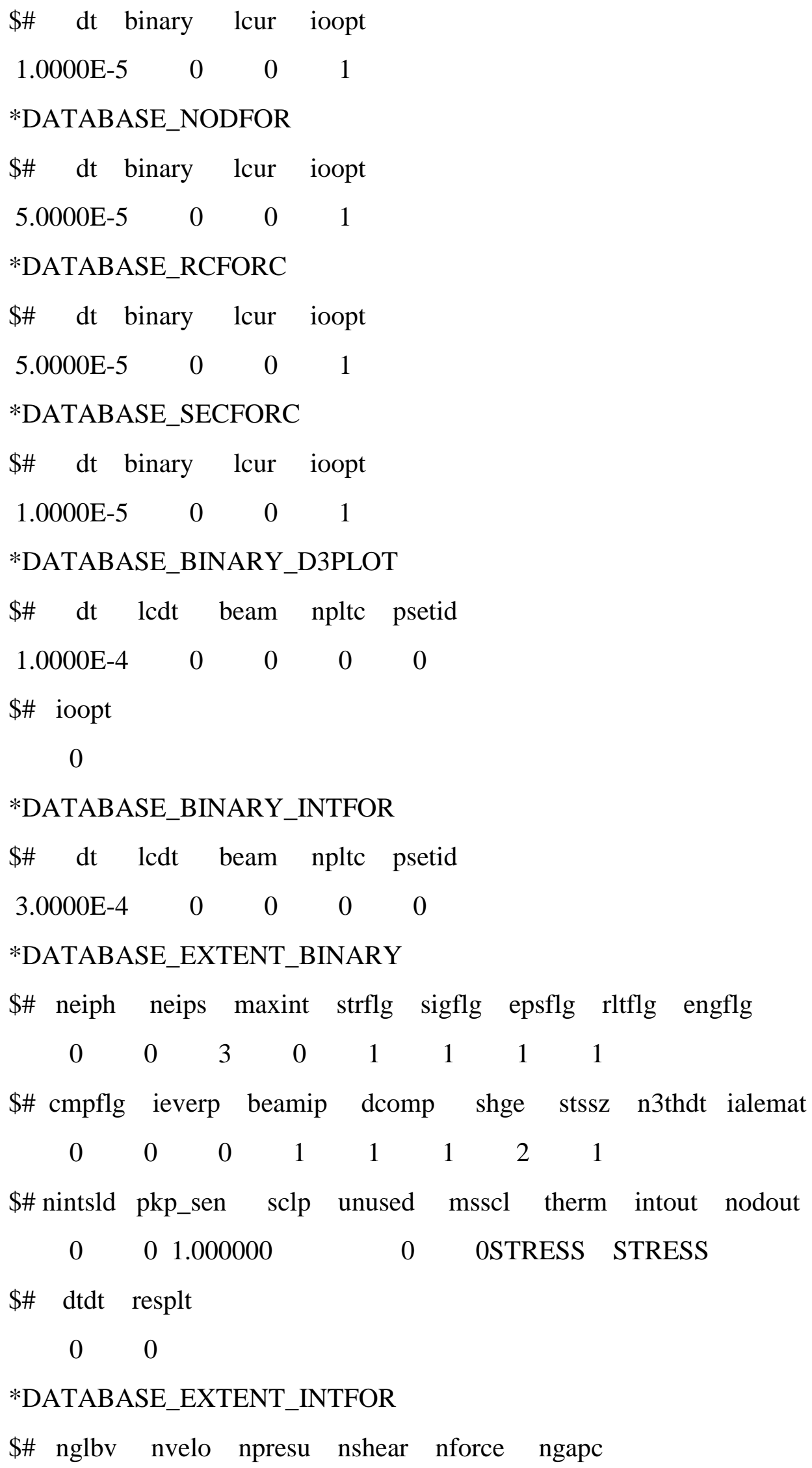


$\begin{array}{llllll}0 & 0 & 0 & 0 & 0 & 1\end{array}$

*BOUNDARY_SPC_SET

\$\# nsid cid dofx dofy dofz dofrx dofry dofrz

$\begin{array}{llllllll}1 & 0 & 1 & 1 & 1 & 1 & 1 & 1\end{array}$

*SET_NODE_LIST_TITLE

NODESET(SPC) 1

\$\# sid da1 da2 da3 da4 solver

$\begin{array}{lllll}1 & 0.000 & 0.000 & 0.000 & 0.000 \mathrm{MECH}\end{array}$

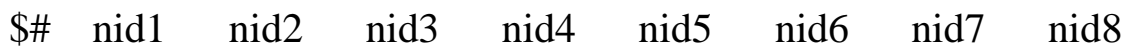

*BOUNDARY_SPC_SET

\$\# nsid cid dofx dofy dofz dofrx dofry dofrz

$\begin{array}{llllllll}2 & 0 & 1 & 0 & 1 & 1 & 1 & 1\end{array}$

*SET_NODE_LIST_TITLE

NODESET(SPC) 2

\$\# sid da1 da2 da3 da4 solver

$\begin{array}{lllll}2 & 0.000 & 0.000 & 0.000 & 0.000 \mathrm{MECH}\end{array}$

$\$ \#$ nid1 nid2 nid3 nid4 nid5 nid6 nid7 nid8

$\begin{array}{llllllll}368146 & 368147 & 368148 & 368149 & 368150 & 368211 & 368212 & 368213\end{array}$

$\begin{array}{llllllll}368214 & 368515 & 368516 & 368517 & 368518 & 368519 & 368520 & 368521\end{array}$

$\begin{array}{llllllll}368522 & 368523 & 368524 & 368525 & 368526 & 368527 & 368528 & 368529\end{array}$

$\begin{array}{llllllll}368530 & 368531 & 368532 & 368533 & 368534 & 0 & 0 & 0\end{array}$

*CONTACT_AUTOMATIC_SURFACE_TO_SURFACE_ID

$\$ \#$ cid title

1Impactor_to_top_0

\$\# ssid msid sstyp mstyp sboxid mboxid spr mpr

$\begin{array}{llllllll}3 & 4 & 3 & 3 & 0 & 0 & 1 & 0\end{array}$

\$\# fs fd dc ve vdc penchk bt dt

$\begin{array}{lllllll}0.000 & 0.000 & 0.000 & 0.000 & 0.000 & 0 & 0.0001 .0000 \mathrm{E}+20\end{array}$

\$\# $\quad$ sfs $\quad$ sfm $\quad$ sst $\quad$ mst $\quad$ sfst $\quad$ sfmt $\quad$ fsf $\quad$ vsf 
$\begin{array}{llllllll}1.000000 & 1.000000 & 0.000 & 0.000 & 1.000000 & 1.000000 & 1.000000 & 1.000000\end{array}$

\$\# soft sofscl lcidab maxpar sbopt depth bsort frcfrq

$\begin{array}{llllllll}0 & 0.000 & 0 & 0.000 & 0.000 & 0 & 0 & 0\end{array}$

\$\# penmax thkopt shlthk snlog isym i2d3d sldthk sldstf

$\begin{array}{llllllll}0.000 & 0 & 0 & 0 & 0 & 0 & 0.000 & 0.000\end{array}$

\$\# igap ignore dprfac dtstif unused unused flangl cid_rcf

$\begin{array}{llllll}2 & 0 & 0.000 & 0.000 & 0.000 & 0\end{array}$

*CONTACT_AUTOMATIC_ONE_WAY_SURFACE_TO_SURFACE_TIEBREAK_ID

\$ cid title

2tiebreak_M46J_TC250

\$\# ssid msid sstyp mstyp sboxid mboxid spr mpr

$\begin{array}{llllllll}1 & 2 & 0 & 0 & 0 & 0 & 1 & 0\end{array}$

$\$ \#$ fs $\quad$ fd $\quad \mathrm{dc} \quad \mathrm{vc}$ vdc penchk bt $\mathrm{dt}$

$\begin{array}{lllllll}0.000 & 0.000 & 0.000 & 0.000 & 0.000 & 0 & 0.0001 .0000 \mathrm{E}+20\end{array}$

$\$ \#$ sfs $\quad$ sfm $\quad$ sst $\quad$ mst $\quad$ sfst $\quad$ sfmt fsf $\quad$ vsf

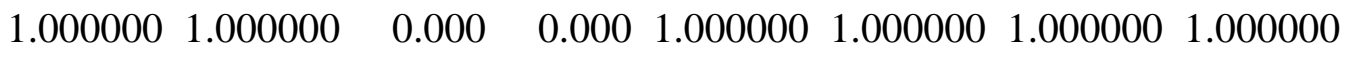

\$\# option nfls sfls param eraten erates ct2cn cn

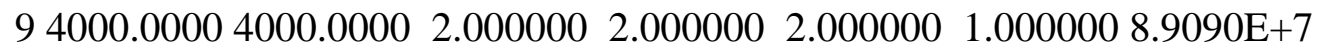

\$\# soft sofscl lcidab maxpar sbopt depth bsort frcfrq

$\begin{array}{llllllll}1 & 0.000 & 0 & 0.000 & 0.000 & 0 & 0 & 0\end{array}$

*SET_SEGMENT_TITLE

top_0_bot

\$\# sid da1 da2 da3 da4 solver

$\begin{array}{lllll}1 & 0.000 & 0.000 & 0.000 & 0.000 \mathrm{MECH}\end{array}$

$\begin{array}{lllllllll}\$ & \mathrm{n} 1 & \mathrm{n} 2 & \mathrm{n} 3 & \mathrm{n} 4 & \mathrm{a} 1 & \mathrm{a} 2 & \mathrm{a} 3 & \mathrm{a} 4\end{array}$

*PART

\$\# title

1 bot_0

$\$ \#$ pid secid mid eosid hgid grav adpopt tmid 


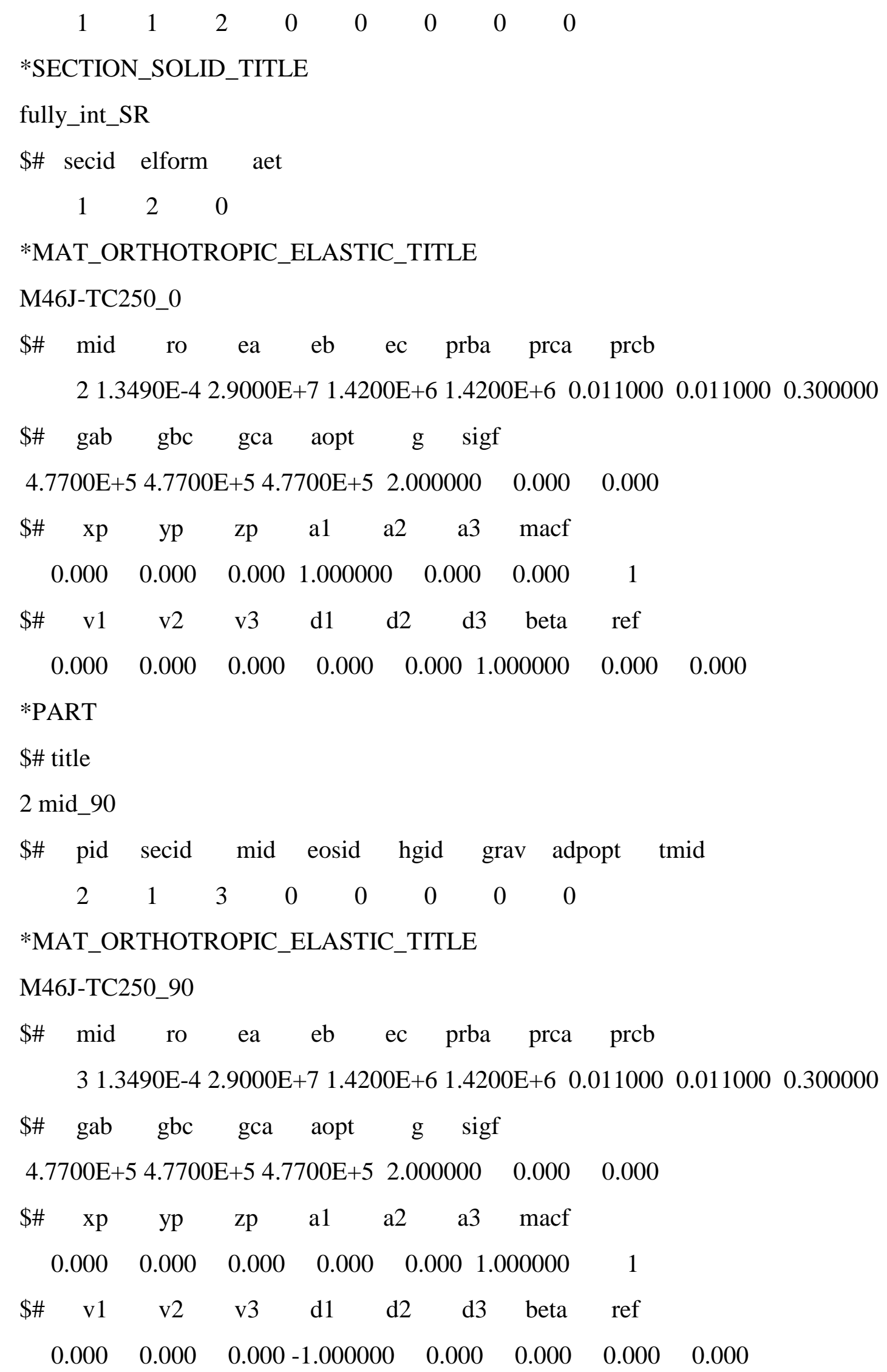




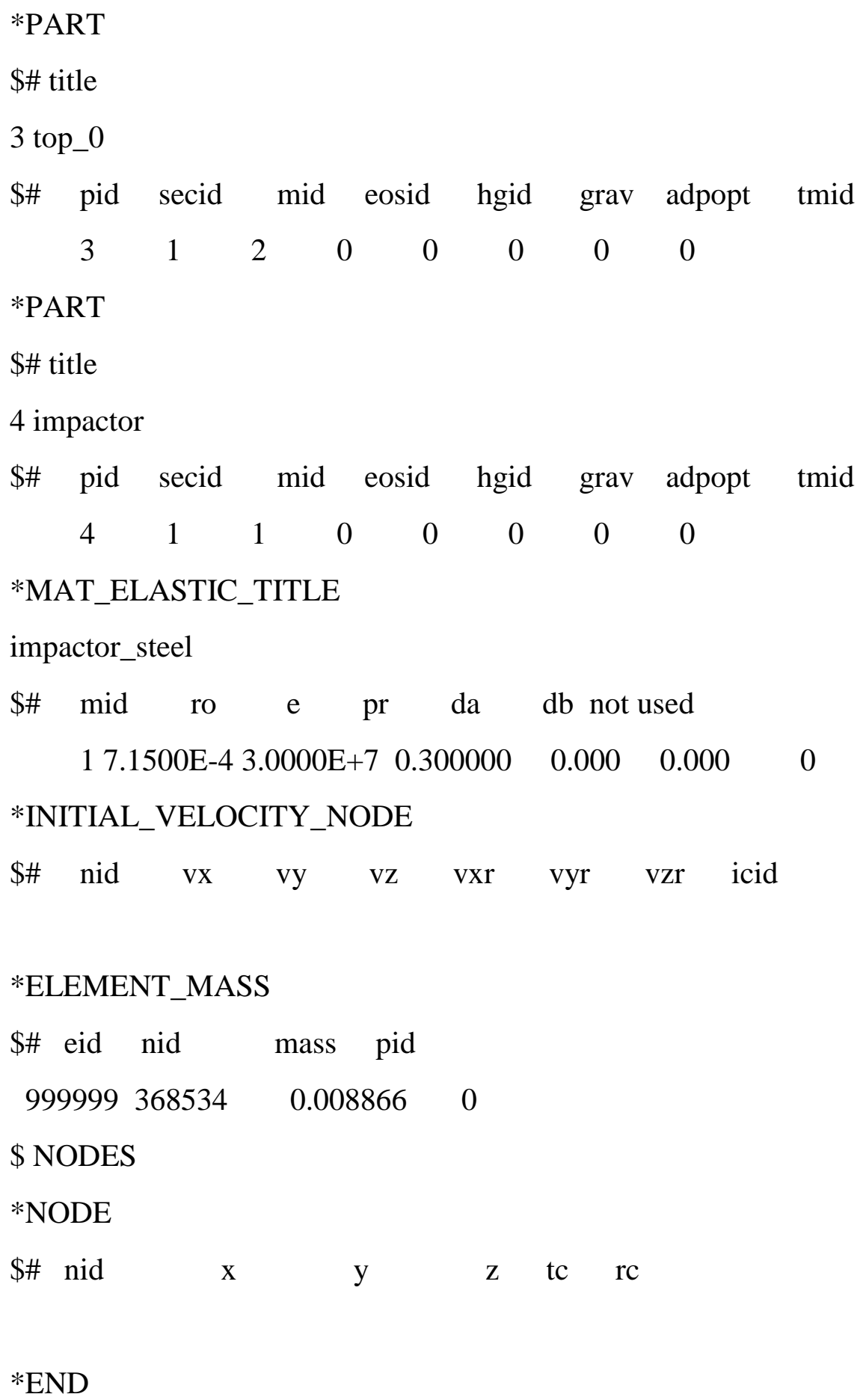




\section{APPENDIX C}

Mode I fracture simulation reduced keyword file

\$\# LS-DYNA Keyword file created by LS-PrePost 4.0 - 28Jan2013(19:00)

\$\# Created on Apr-04-2016 (11:43:19)

*KEYWORD

*TITLE

\$\# title

LS-DYNA keyword deck by LS-PrePost

*CONTROL_ACCURACY

\$\# osu inn pidosu

$\begin{array}{lll}0 & 4 & 0\end{array}$

*CONTROL_ENERGY

\$\# hgen rwen slnten rylen

$\begin{array}{llll}2 & 2 & 1 & 1\end{array}$

*CONTROL_TERMINATION

\$\# endtim endcyc dtmin endeng endmas

$$
\begin{array}{lllll}
0.050000 & 0 & 0.000 & 0.000 & 0.000
\end{array}
$$

*CONTROL_TIMESTEP

\$\# dtinit tssfac isdo tslimt dt2ms lctm erode ms1st

$\begin{array}{llllllll}3.0000 \mathrm{E}-8 & 0.800000 & 0 & 0.000 & 0.000 & 0 & 0 & 0\end{array}$

\$\# dt2msf dt2mslc imscl unused unused $\mathrm{rmscl}$

$\begin{array}{llll}0.000 & 0 & 0 & 0.000\end{array}$

*DATABASE_ATDOUT

\$\# dt binary lcur ioopt

3.0000E-6 $0 \quad 0 \quad 1$

*DATABASE_GLSTAT

\$\# dt binary lcur ioopt

$1.0000 \mathrm{E}-5 \quad 0 \quad 0 \quad 1$

*DATABASE_MATSUM 


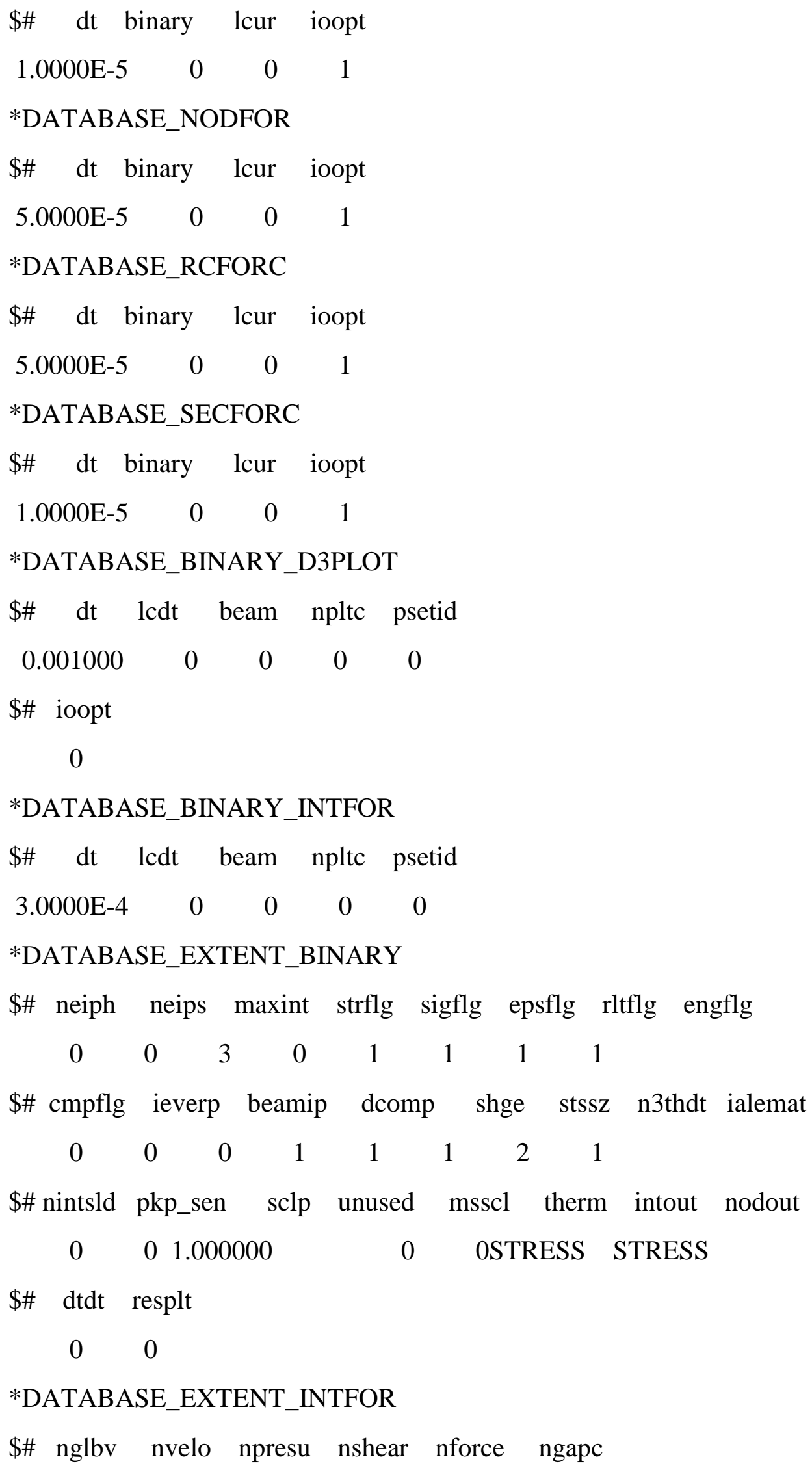




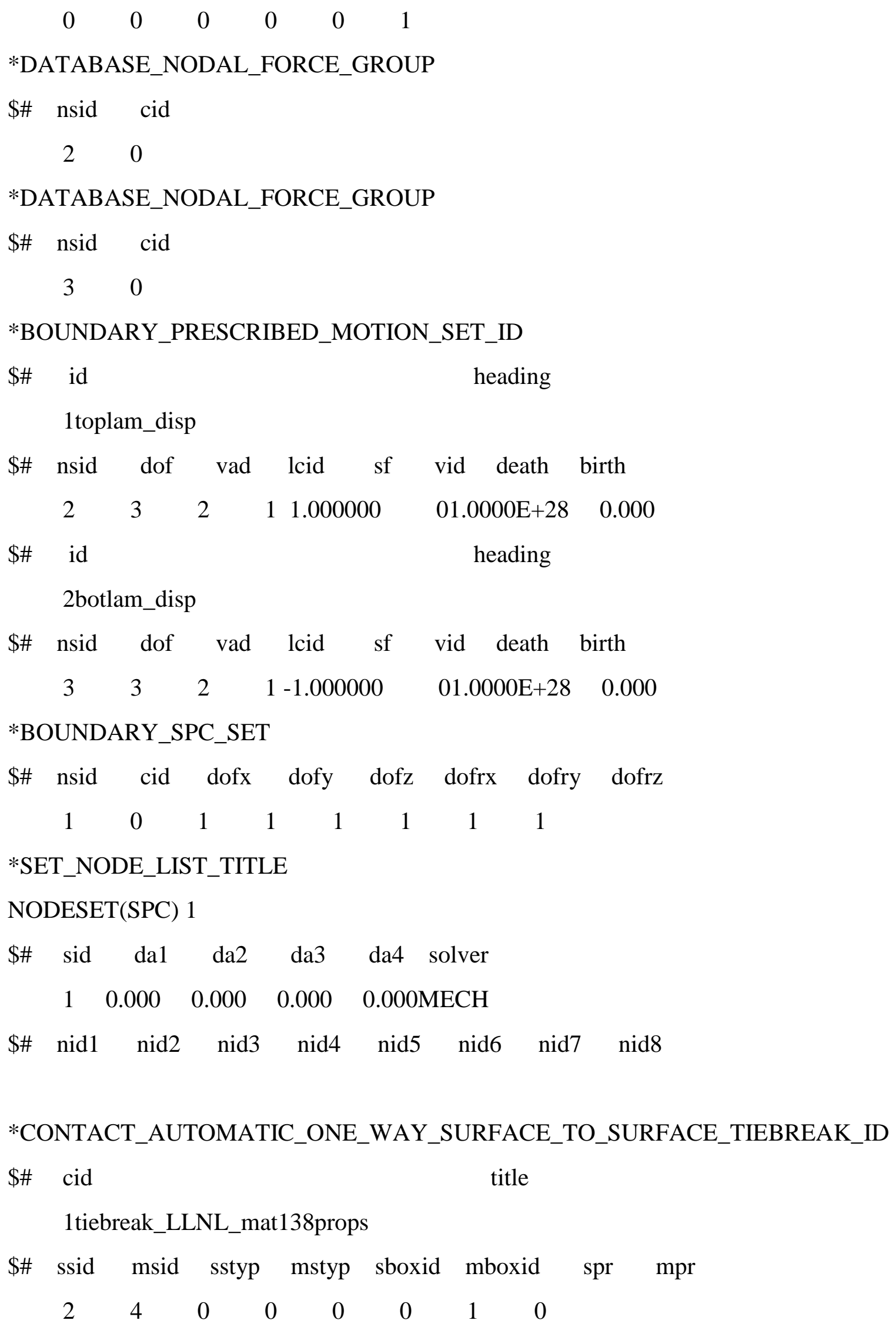


$\$$ fs fd $\mathrm{dc} \quad \mathrm{vc} \quad \mathrm{vdc}$ penchk bt $\mathrm{dt}$ $\begin{array}{lllllll}0.000 & 0.000 & 0.000 & 0.000 & 0.000 & 0 & 0.0001 .0000 \mathrm{E}+20\end{array}$

$\$ \#$ sfs sfm sst mst sfst sfmt fsf vsf

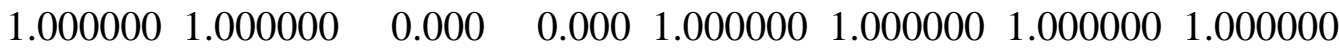

\$\# option nfls sfls param eraten erates ct2cn cn $94000.00004000 .00002 .000000 \quad 0.742300 \quad 0.742300 \quad 1.000000 \quad 8.9090 \mathrm{E}+7$

\$\# soft sofscl lcidab maxpar sbopt depth bsort frcfrq $\begin{array}{llllllll}1 & 0.000 & 0 & 0.000 & 0.000 & 0 & 0 & 0\end{array}$

*SET_SEGMENT_TITLE

toplam_tiebreak

\$\# $\quad$ sid da1 da2 da3 da4 solver

$\begin{array}{lllll}2 & 0.000 & 0.000 & 0.000 & 0.000 \mathrm{MECH}\end{array}$

$\begin{array}{lllllllll}\$ \# & \mathrm{n} 1 & \mathrm{n} 2 & \mathrm{n} 3 & \mathrm{n} 4 & \mathrm{a} 1 & \mathrm{a} 2 & \mathrm{a} 3 & \mathrm{a} 4\end{array}$

*SET_SEGMENT_TITLE

botlam_tiebreak

\$\# sid da1 da2 da3 da4 solver

$\begin{array}{lllll}4 & 0.000 & 0.000 & 0.000 & 0.000 \mathrm{MECH}\end{array}$

$\begin{array}{lllllllll}\$ & \mathrm{n} 1 & \mathrm{n} 2 & \mathrm{n} 3 & \mathrm{n} 4 & \mathrm{a} 1 & \mathrm{a} 2 & \mathrm{a} 3 & \mathrm{a} 4\end{array}$

*CONTACT_AUTOMATIC_SURFACE_TO_SURFACE_ID

\$ cid title

2delam_contact

\$ ssid msid sstyp mstyp sboxid mboxid spr mpr $\begin{array}{llllllll}1 & 3 & 0 & 0 & 0 & 0 & 1 & 0\end{array}$

$\$ \#$ fs fd dc vc vdc penchk bt $\mathrm{dt}$ $\begin{array}{lllllll}0.000 & 0.000 & 0.000 & 0.000 & 0.000 & 0 & 0.0001 .0000 \mathrm{E}+20\end{array}$

$\$$ \$ $\quad$ sfs $\quad$ sfm $\quad$ sst $\quad$ mst $\quad$ sfst sfmt fsf vsf

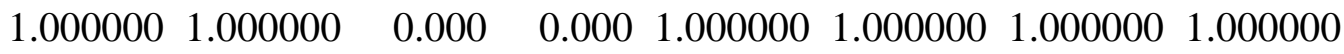

\$\# soft sofscl lcidab maxpar sbopt depth bsort frcfrq 


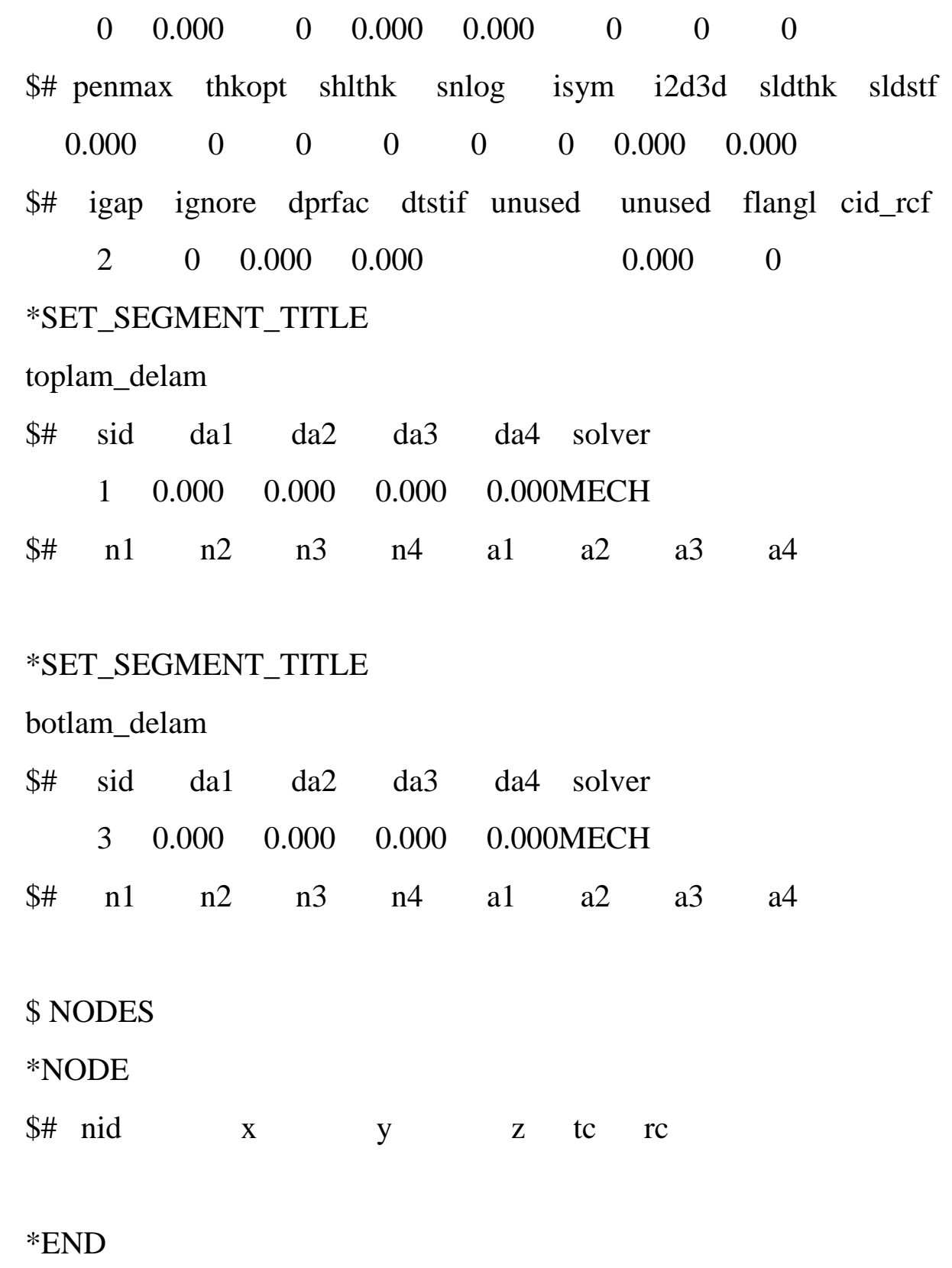




\section{APPENDIX D}

\section{Mode I fracture simulation eigenvalue reduced keyword file}

\$\# LS-DYNA Keyword file created by LS-PrePost 4.0 - 28Jan2013(19:00)

\$\# Created on May-02-2016 (17:43:40)

*KEYWORD

*TITLE

\$\# title

LS-DYNA keyword deck by LS-PrePost

*CONTROL_ACCURACY

\$\# osu inn pidosu

$\begin{array}{lll}0 & 4 & 0\end{array}$

*CONTROL_ENERGY

\$\# hgen rwen slnten rylen

$\begin{array}{llll}2 & 2 & 1 & 1\end{array}$

*CONTROL_TERMINATION

\$\# endtim endcyc dtmin endeng endmas

$\begin{array}{lllll}0.050000 & 0 & 0.000 & 0.000 & 0.000\end{array}$

*CONTROL_TIMESTEP

\$\# dtinit tssfac isdo tslimt dt2ms lctm erode ms1st

$\begin{array}{llllllll}3.0000 \mathrm{E}-8 & 0.800000 & 0 & 0.000 & 0.000 & 0 & 0 & 0\end{array}$

\$\# dt2msf dt2mslc imscl unused unused rmscl

$$
\begin{array}{llll}
0.000 & 0 & 0 & 0.000
\end{array}
$$

*DATABASE_ATDOUT

\$\# dt binary lcur ioopt

3.0000E-6 $\quad 0 \quad 0 \quad 1$

*DATABASE_GLSTAT

\$ dt binary lcur ioopt

$1.0000 \mathrm{E}-5 \quad 0 \quad 0 \quad 1$

*DATABASE_MATSUM 


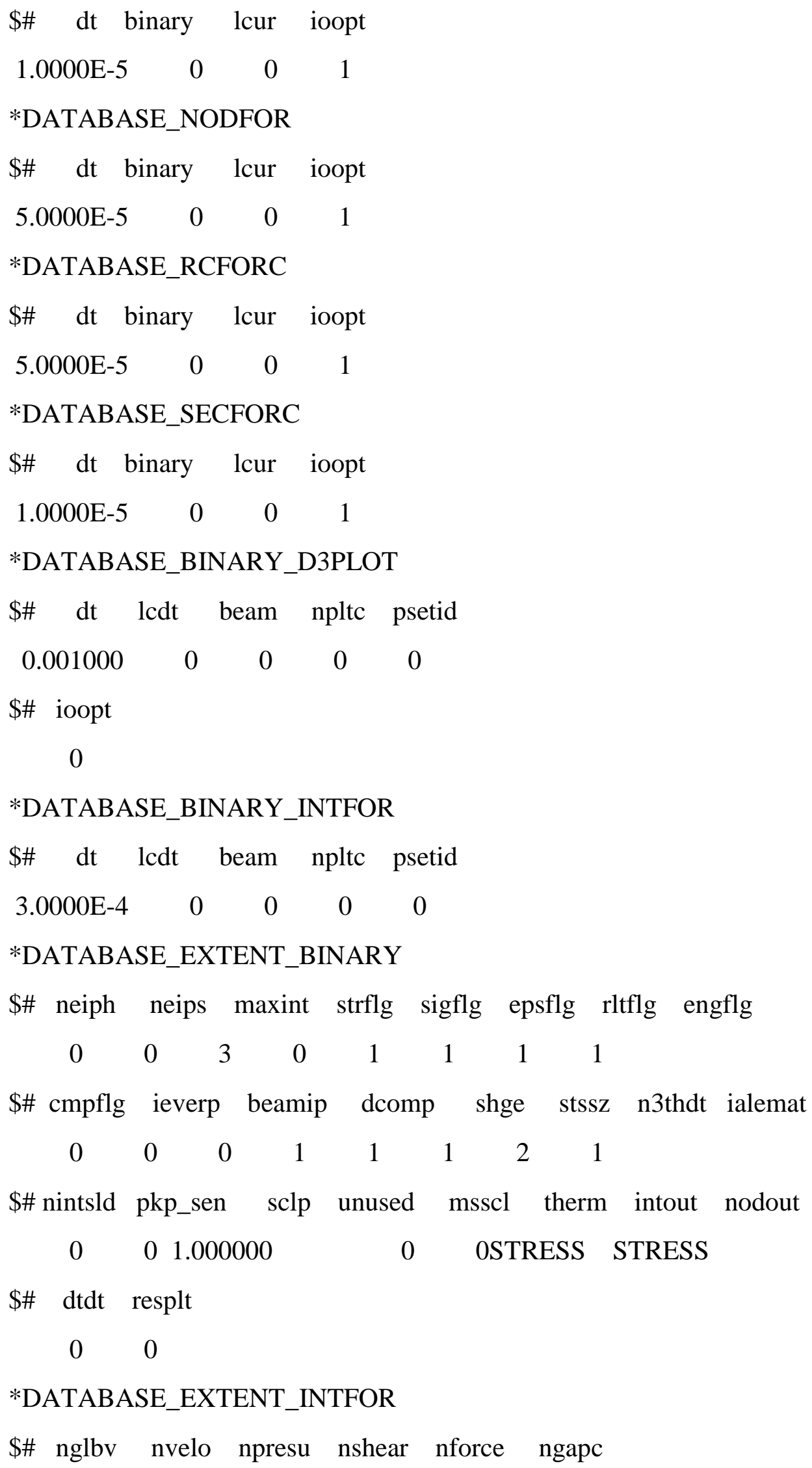




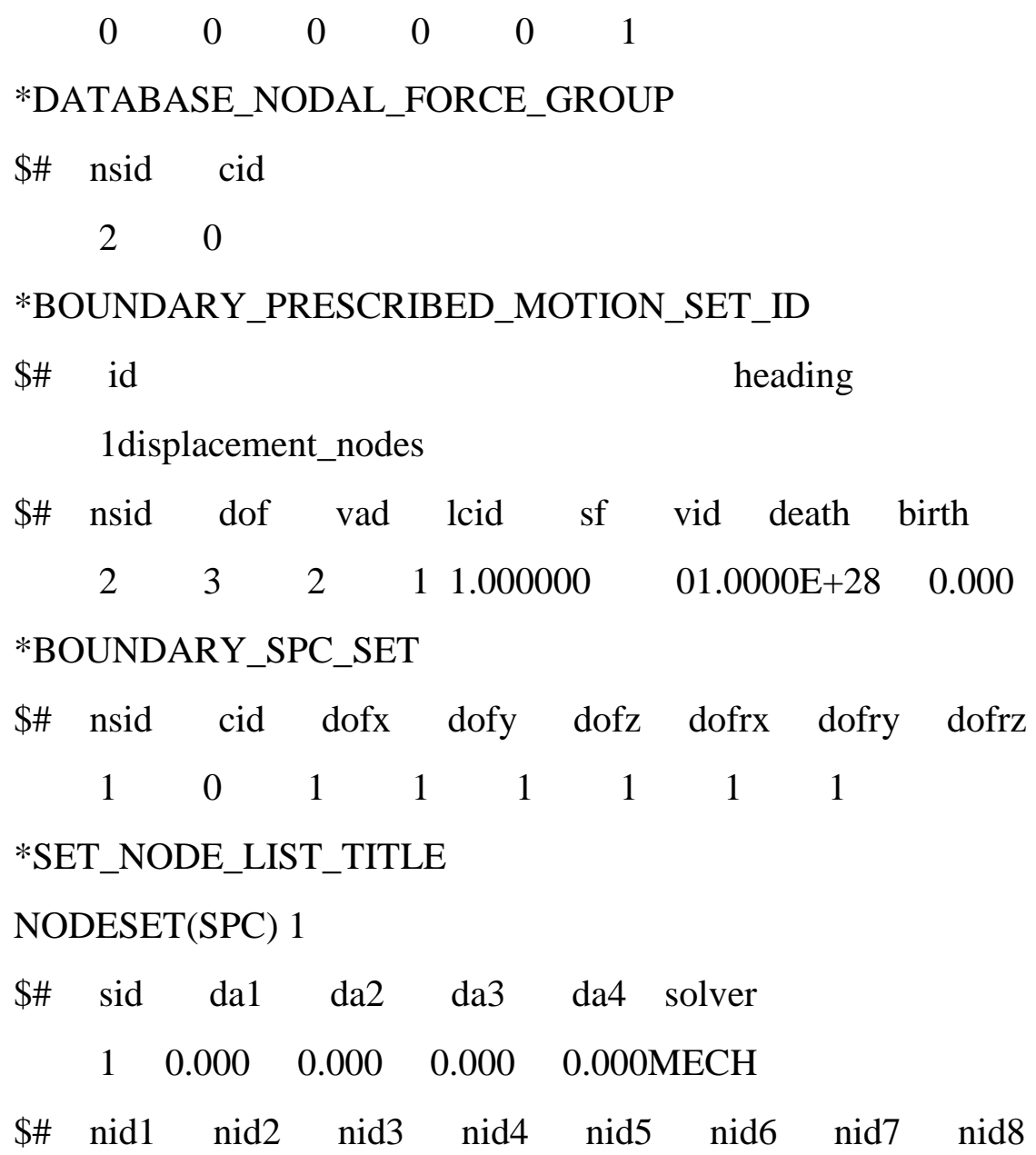


$1 \quad 1.4500 \mathrm{E}-4$ 7.5000E+6 $1.2800 \mathrm{E}+6 \quad 1.2800 \mathrm{E}+6 \quad 0.025450 \quad 0.025450 \quad 0.300000$

\$\# gab gbc gca aopt $\mathrm{g}$ sigf

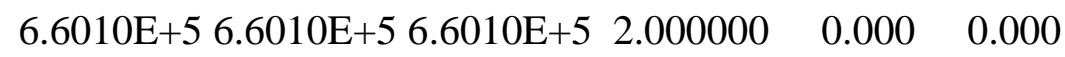

$\$ \# \quad x p \quad$ yp $\quad$ zp $\quad$ a1 a2 a3 macf

$\begin{array}{lllllll}0.000 & 0.000 & 0.000 & 1.000000 & 0.000 & 0.000 & 1\end{array}$

$\$ \# \quad v 1 \quad v 2 \quad v 3 \quad d 1 \quad d 2 \quad d 3$ beta ref

$\begin{array}{llllllll}0.000 & 0.000 & 0.000 & 0.000 & 1.000000 & 0.000 & 0.000 & 0.000\end{array}$

*DEFINE_CURVE_TITLE

10_in_per_sec

\$\# lcid sidr sfa sfo offa offo dattyp

$\begin{array}{lllllll}1 & 0 & 1.000000 & 1.000000 & 0.000 & 0.000 & 0\end{array}$

$\$ \# \quad$ a1 $\quad$ o1

$0.000 \quad 0.000$

$0.050000 \quad 0.500000$

$0.200000 \quad 0.500000$

*SET_NODE_LIST_TITLE

displacement_nodes

\$ $\quad$ sid $\quad$ da1 da2 da3 da4 solver

$\begin{array}{lllll}2 & 0.000 & 0.000 & 0.000 & 0.000 \mathrm{MECH}\end{array}$

$\$ \#$ nid1 nid2 nid3 nid4 nid5 nid6 nid7 nid8

*ELEMENT_SOLID

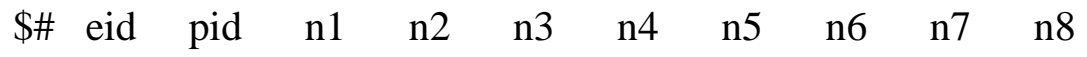

*NODE

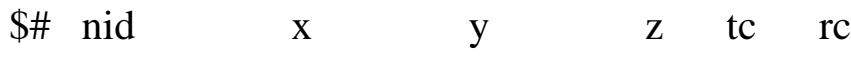

*END 


\section{APPENDIX E}

Force vs. time experimental impact results

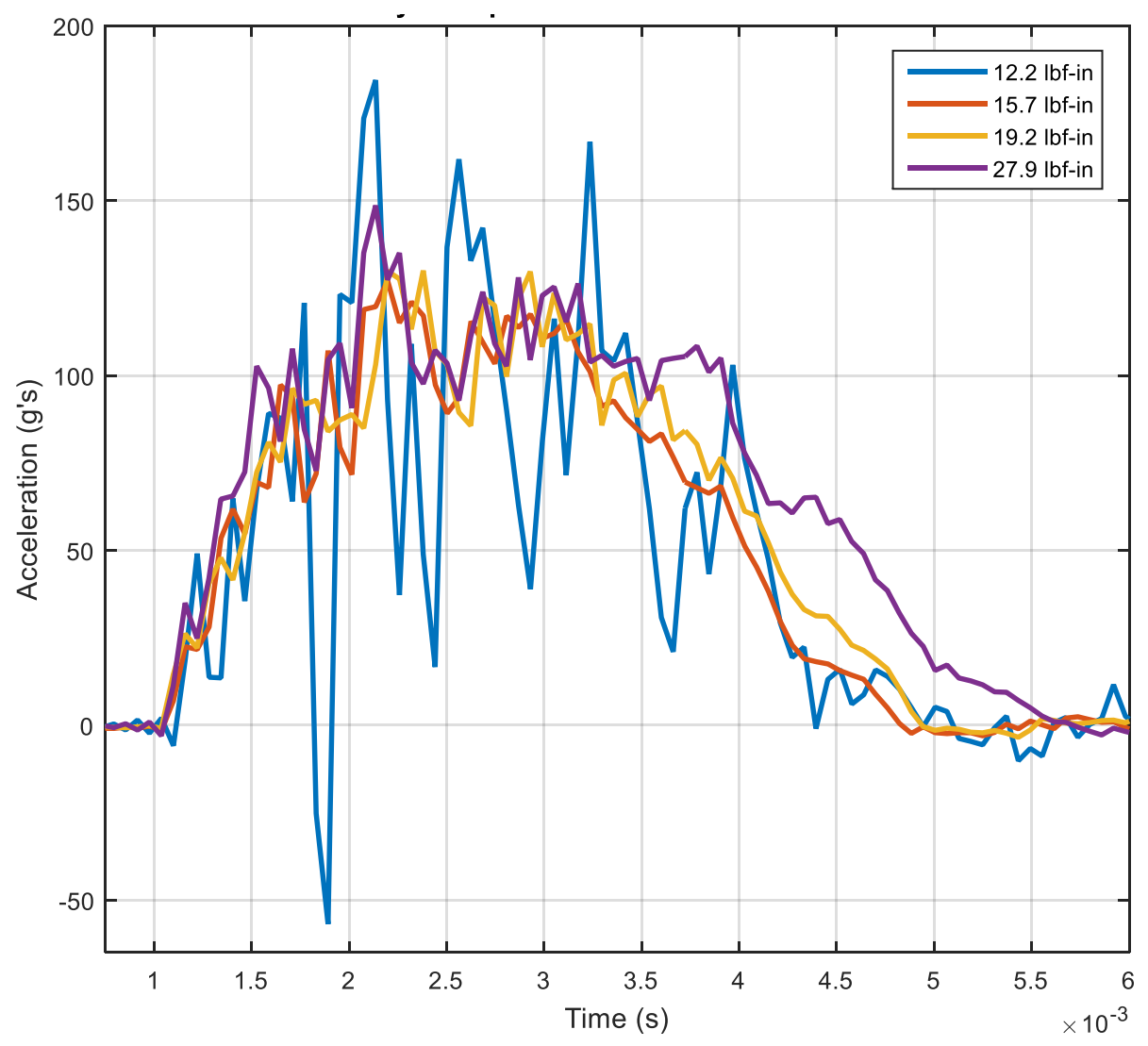

Figure 46 Overlaid experimental acceleration data for various impact energies 


\section{APPENDIX F}

\section{Vibrothermography experimental results}

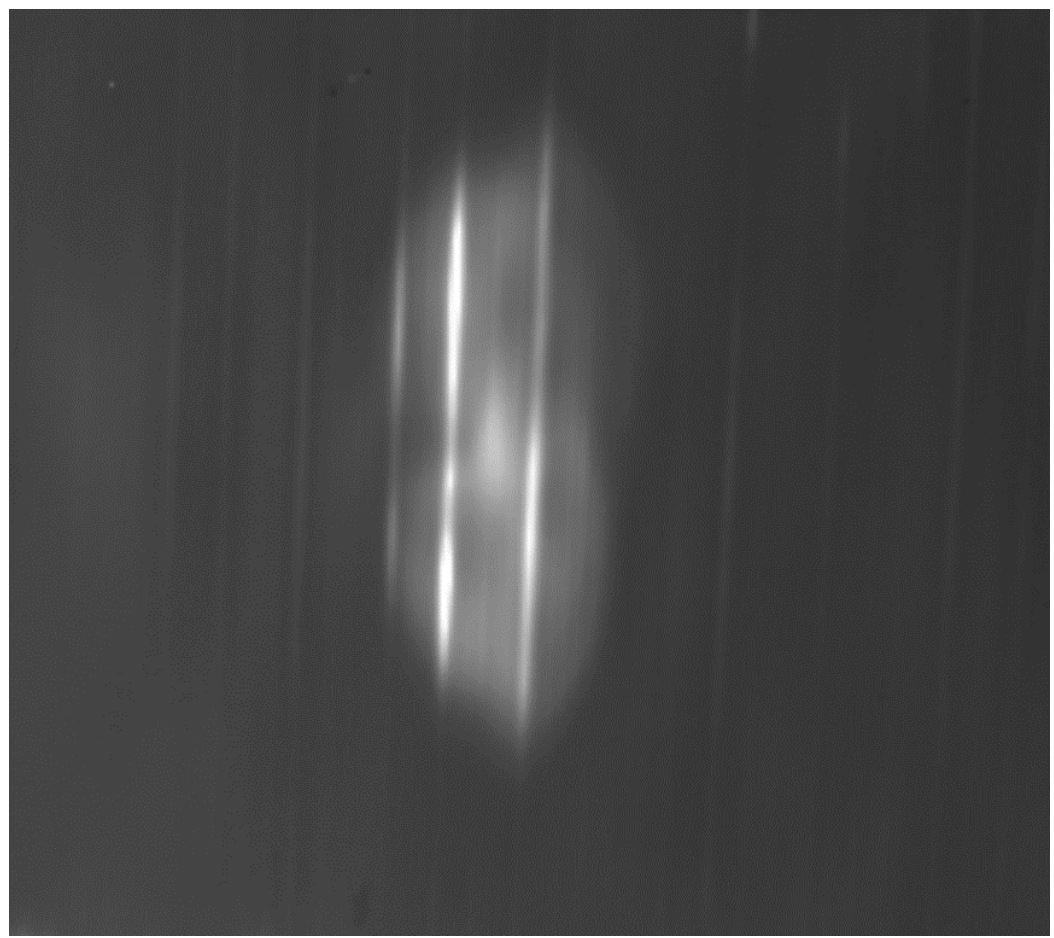

Figure 47 Backside view of $\left[0_{4} / 90_{8} / 0_{4}\right]$ laminate from $12.2 \mathrm{lb}_{\mathrm{f}}$-in impact energy

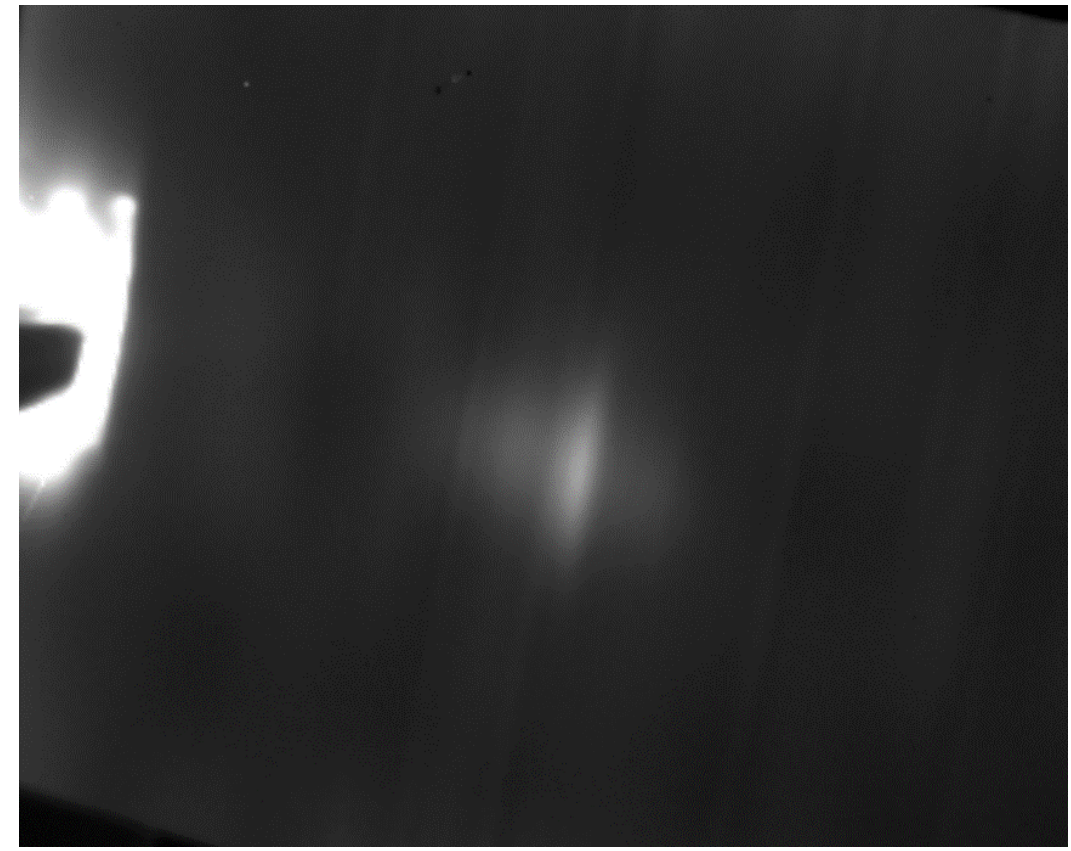

Figure 48 Impact side view of $\left[0_{4} / 90_{8} / 0_{4}\right]$ laminate from $12.2 \mathrm{lb}_{\mathrm{f}}$-in impact energy 


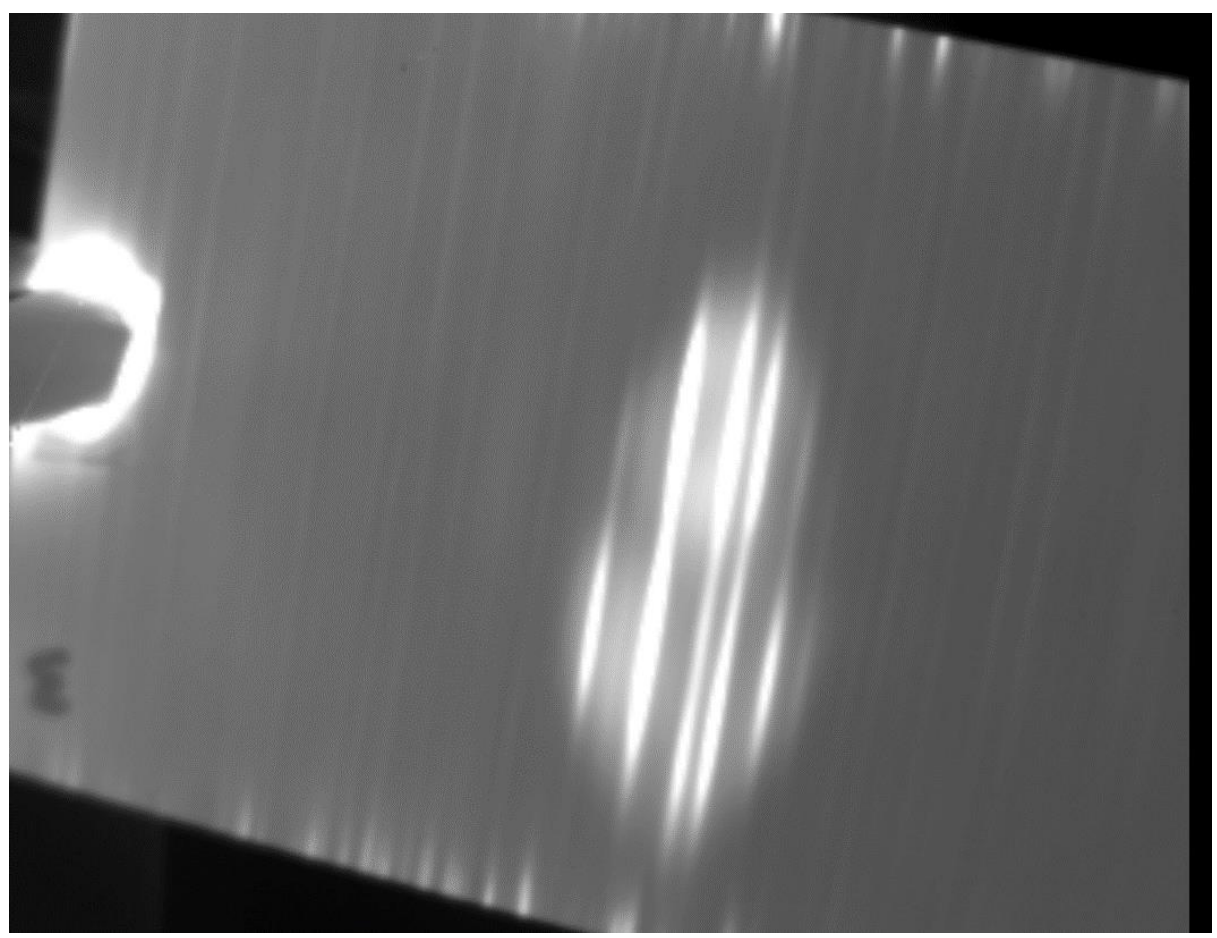

Figure 49 Backside view of $\left[0_{4} / 90_{8} / 0_{4}\right]$ laminate from $15.7 \mathrm{lb}_{\mathrm{f}}$-in impact energy

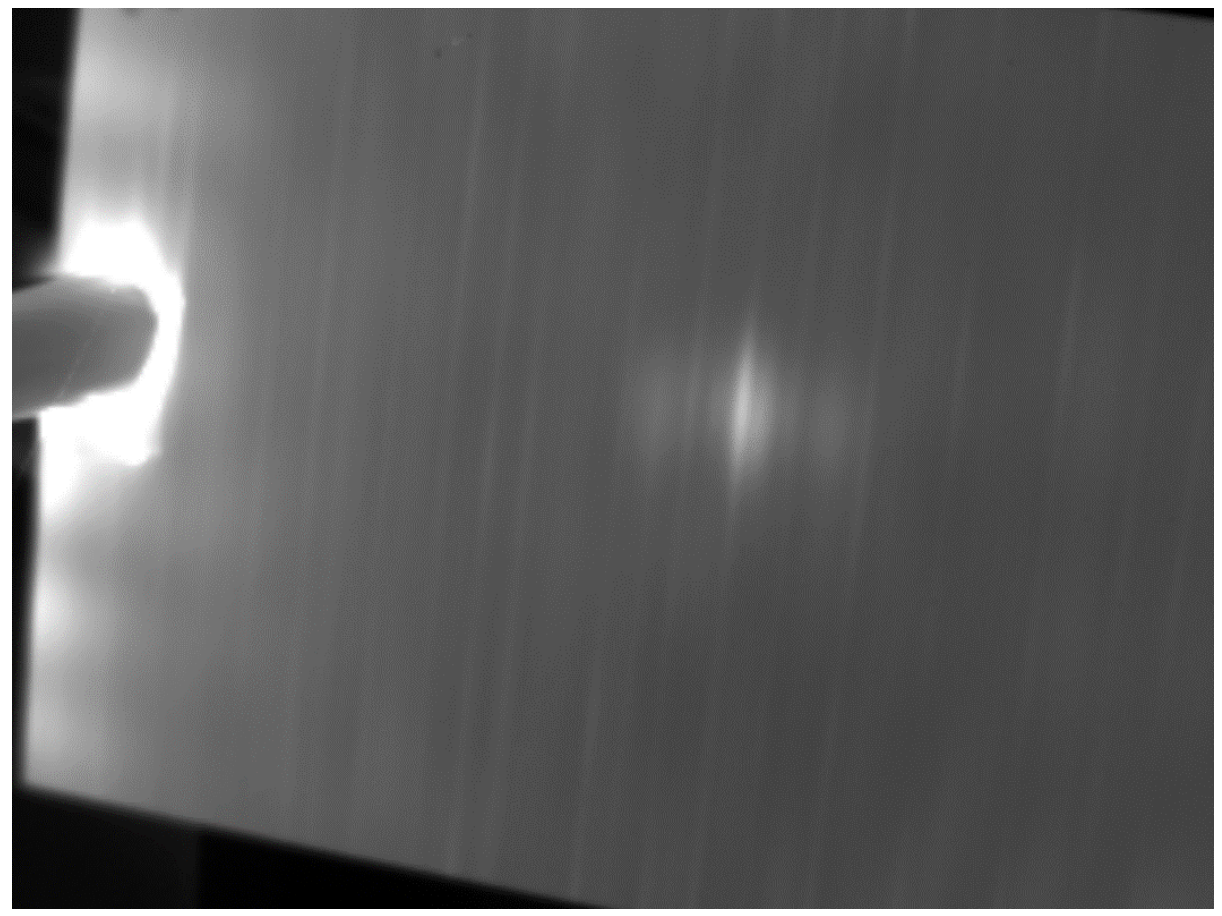

Figure 50 Impact side view of $\left[0_{4} / 90_{8} / 0_{4}\right]$ laminate from $15.7 \mathrm{lb}_{\mathrm{f}}$-in impact energy 


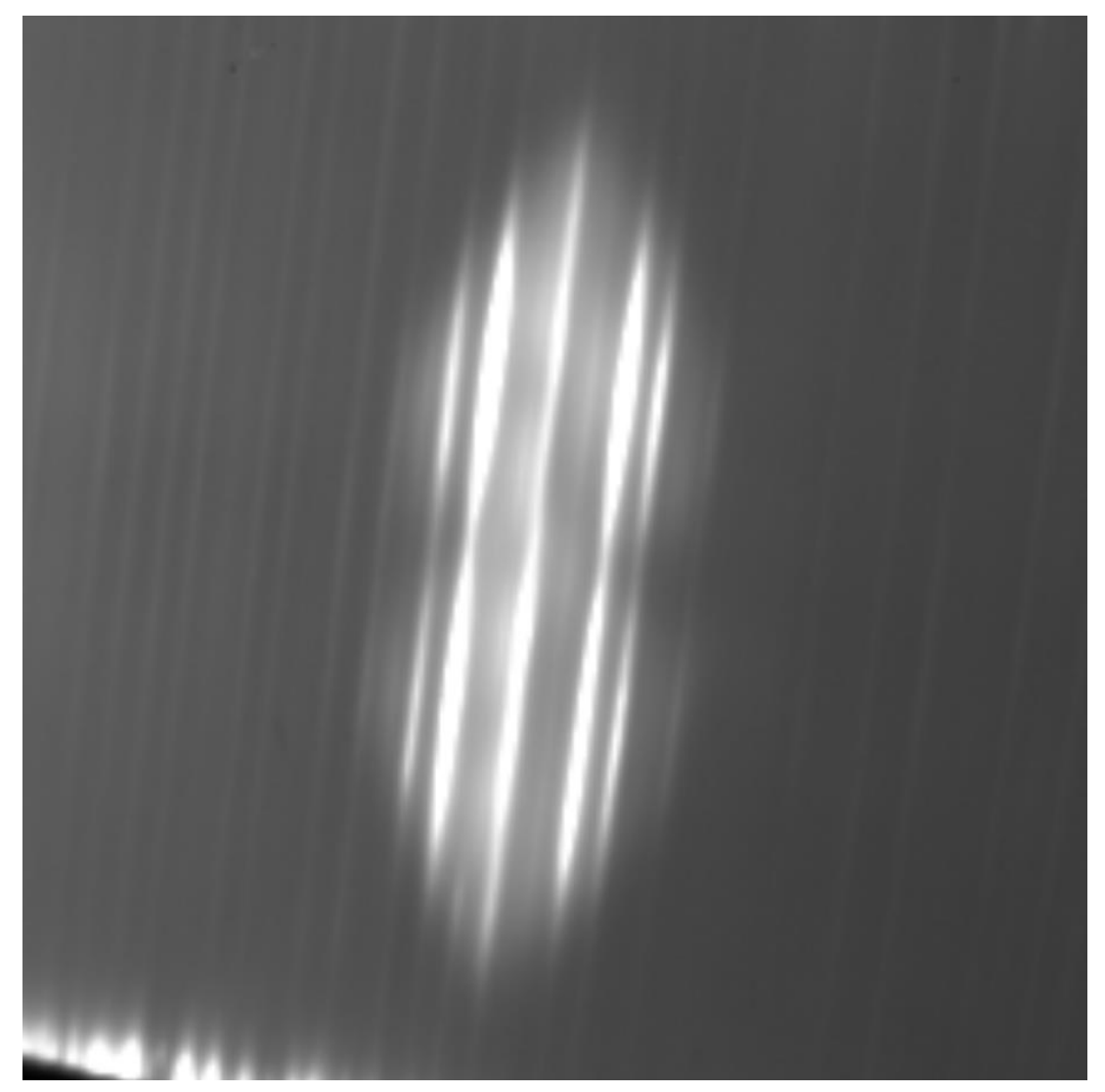

Figure 51 Backside view of $\left[0_{4} / 90_{8} / 0_{4}\right]$ laminate from $19.2 \mathrm{lb}_{\mathrm{f}}$-in impact energy

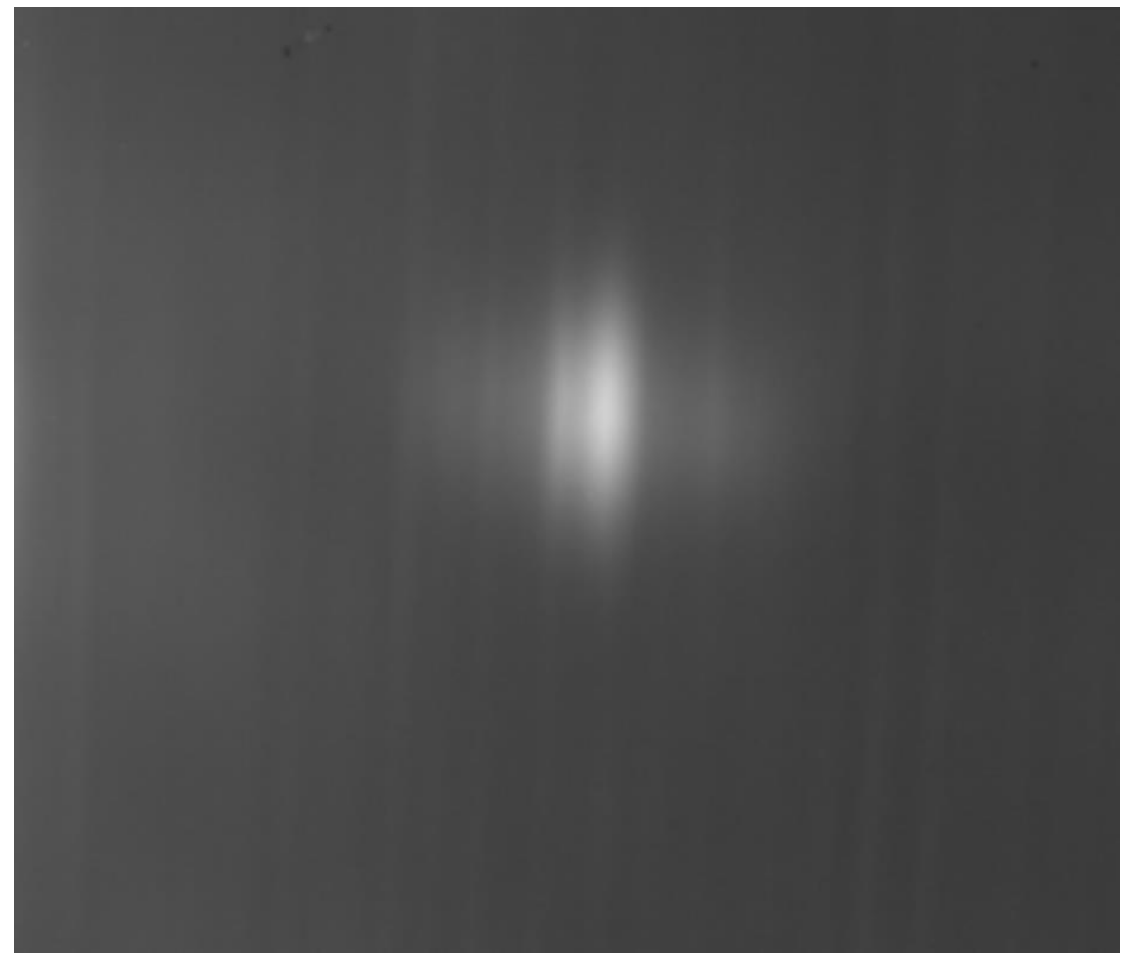

Figure 52 Impact side view of $\left[0_{4} / 90_{8} / 0_{4}\right]$ laminate from $19.2 \mathrm{lb}_{\mathrm{f}}$-in impact energy 


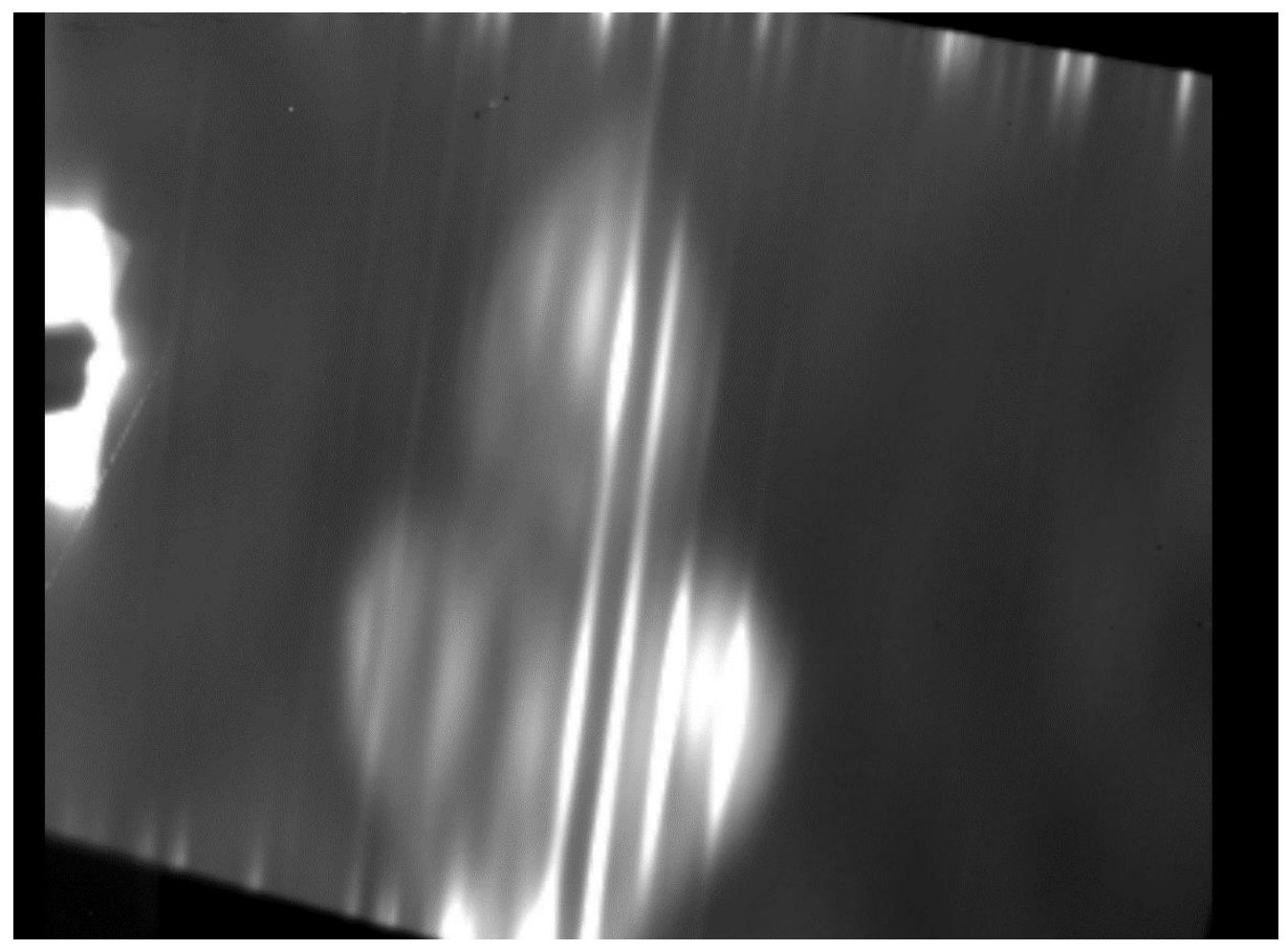

Figure 53 Backside view of $\left[0_{4} / 90_{8} / 0_{4}\right]$ laminate from $27.9 \mathrm{lb}$-in impact energy

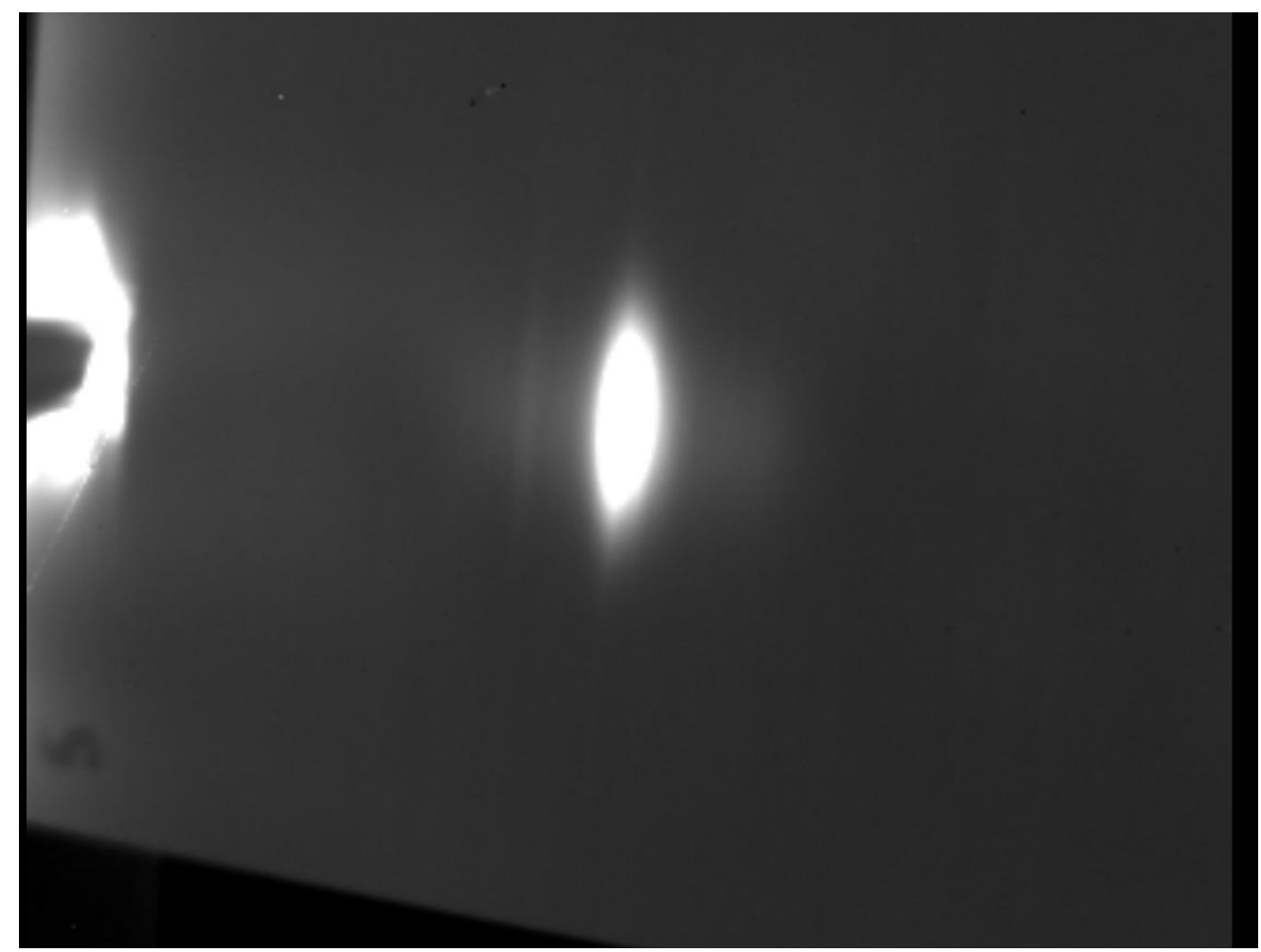

Figure 54 Impact side view of $\left[0_{4} / 90_{8} / 0_{4}\right]$ laminate from $27.9 \mathrm{lb}_{\mathrm{f}}$-in impact energy 